\title{
Interdisziplinäre Grundlagen aus Pädagogik, Informatik und Architektur
}

Die vorliegende Arbeit behandelt die Gestaltung von intelligenten und hybriden Lernräumen durch Anwendung des Internet der Dinge auf Lehr- und Lernprozesse. Um den Forschungskontext zu fokussieren, konzentriert sich die Untersuchung auf den Anwendungsbereich der betrieblichen Weiterbildung mit einem Schwerpunkt auf arbeitsplatzbezogenes Lernen.

Die gestaltungsorientierte Bildungsforschung bildet die theoretische Untermauerung der Arbeit. Das vorliegende Kapitel soll die inhaltlichen Grundlagen für die hierauf aufbauenden Teile der Untersuchung legen. Im Folgenden werden daher Forschungsobjekt, Forschungskontext sowie die angewendete Theorie begrifflich dekonstruiert sowie die grundlegenden theoretischen Bezüge untereinander herausgearbeitet.

Zunächst wird im Unterkapitel 2.1 ein aktueller Stand der Weiterbildungsforschung rezipiert, um darauf aufbauend auf das arbeitsplatzbezogene Lernen als eine spezifische Ausprägung von Lehr- und Lernprozessen in Organisationen zu fokussieren. Dabei wird das arbeitsplatzbezogene Lernen als Begriff und als Gegenstand der Forschung erläutert. Im Anschluss daran werden verschiedene Ansätze und theoretische Hintergründe des arbeitsplatzbezogenen Lernens dargestellt und im Kontext eines persönlichen Wissensmanagements mittels persönlicher Lernumgebungen beleuchtet. Dabei werden auch relevante Mischformen des Lernens wie informelles, formales, selbstgesteuertes, kollaboratives und lebenslanges Lernen betrachtet und schließlich mit den Anforderungen an adaptive und ubiquitäre Lernsettings innerhalb intelligenter und hybrider Lernräume verbunden.

In Abschnitt 2.2 erfolgt eine differenzierte Erörterung des Begriffes Internet der Dinge, wobei auf die Historie und Besonderheiten verwiesen werden. Weiterhin wird auf die verschiedenen Anwendungsfelder des Internet der Dinge eingegangen und eine Erweiterung auf den Bildungskontext diskutiert. 
Hierauf basierend führt Unterkapitel 2.3 Lernräume als Forschungsgegenstand der vorliegenden Arbeit ein. Hierbei werden raumsoziologische Grundlagen zunächst in einem theoretischen Rahmen dargestellt und die Konzepte intelligenter und hybrider Lernräume auf Basis der vorhergehenden Abschnitte hergeleitet, dekonstruiert und begrifflich wie inhaltlich zunächst abgegrenzt und letztlich miteinander verwoben. Anschließend folgt ein Überblick verschiedener Büroraumgestaltungskonzepte, die im Sinne intelligenter und hybrider Lernräume mit smarten Applikationen angereichert werden. In diesem Zusammenhang werden Funktionalitäten und mögliche Einsatzfelder intelligenter Lernräume skizziert und die Brücke zum arbeitsplatzbezogenem Lernen geschlagen. Aufgrund der noch jungen Entwicklungsgeschichte des Technologiekonstruktes Internet der Dinge bzw. intelligenter und hybrider Lernräume wird in Abschnitt 2.3.4 das Thema Datenschutz und Datensicherheit thematisiert und in einen bildungstechnologischen Zusammenhang gebracht.

Abschnitt 2.4 synthetisiert den interdisziplinären Forschungsstand in der Einführung des Begriffes „Smart Learning Environments“ und argumentiert, welchen Zusammenhang es zum Internet der Dinge gibt. Schließlich wird in Unterkapitel 2.4.1 begründet, warum im Folgenden Smart Learning Environments als Forschungsgegenstand der Arbeit zu betrachten sind. Darauf aufbauend folgt die Darstellung der Forschungslücken.

Das Kapitel schließt in 2.5 mit einer aus den Grundlagen abgeleiteten Diskussion zum Untersuchungsgegenstand intelligenter und hybrider Lernräume und formuliert zusammenfassend Herausforderung sowie Konsequenzen, die sich hieraus für das Forschungsdesign der interdisziplinären Arbeit ergeben.

\subsection{Empirische Weiterbildungsforschung}

Lehr- und Lernprozesse nehmen eine zentrale Rolle in der vorliegenden Arbeit ein. Um eine fundierte bildungswissenschaftliche Grundlage für die nachkommenden Kapitel zu entwickeln, werden in den folgenden Abschnitten aktuelle empirische Befunde aus der Lehr- und Lernforschung der Erwachsenenbildung (Weiterbildungsforschung) diskutiert und relevante theoretische Bezüge zur Forschungsarbeit abgeleitet. Ergänzt werden die Befunde der Weiterbildungsforschung durch Erkenntnisse aus angrenzenden Disziplinen, wie beispielsweise der pädagogischen Psychologie oder der Neurowissenschaften.

Da sich die Forschungsarbeit im Speziellen auf arbeitsplatzbezogenes Lernen fokussiert, werden in einem finalen Schritt Anforderungen an die Gestaltung von Lehr- und Lernprozessen destilliert und systematisch in einem direkten Bezug 
zu formalen Lehrprozessen der betrieblichen Weiterbildung sowie in Bezug zum Lernen am Arbeitsplatz betrachtet. Normative und konzeptionelle Annahmen werden zusammen mit den empirischen Befunden der Lehr- und Lernforschung expliziert und bilden eine theoretische Rahmung für die Forschungsarbeit. Dabei soll zunächst der Begriff des arbeitsplatzbezogenen Lernens in seiner Bedeutung dekonstruiert und Bezüge zu anderen Lernformen hergestellt werden. Darüber hinaus werden unterschiedliche Ausprägungen und Rahmenbedingungen sowie Befunde zum arbeitsplatzbezogenen Lernen aus Perspektive der empirischen Lernforschung dargestellt, um konzeptionelle Annahmen zu stützen und eine fundierte theoretische Grundlage für die folgenden Kapitel zu konstituieren.

\subsubsection{Erwachsenenpädagogische Einordnung}

In der Erwachsenenbildung als Teildisziplin der Erziehungswissenschaft gibt es drei anerkannte Lerntheorien, die Erklärungsmodelle für das menschliche Lehren und Lernen liefern.

Der Behaviorismus nach Thorndike, Watson, Skinner und Pawlow verfolgt dabei den Ansatz der klassischen/operanten Konditionierung, bei welchem Lernen als Ergebnis aus Stimulus und Response hervorgerufen wird. Die Lernenden selbst werden dabei aus behavioristischer Perspektive als Black-Box betrachtet.

In Abgrenzung zum Behaviorismus stehen beim theoretischen Ansatz des Kognitivismus nach Bruner, Lewin und Piaget, die individuellen Informationsverarbeitungsprozesse der Lernenden im Mittelpunkt. Darin wird der Mensch als abgeschlossenes, informationsverarbeitendes System betrachtet, das Informationen kodiert, speichert, transformiert und abruft. Dieser Paradigmenwechsel wurde durch die kognitive Wende ausgelöst. Kognitivistische Lerntheorien gehen davon aus, dass Lernen durch komplexe innere Prozesse beeinflusst wird, die zwischen Reiz und Reaktion liegen. Aus kognitivistischer Perspektive sind die Lernenden selbstgesteuerte Wesen, die durch kognitive Denk- und Verstehensprozesse lernen. Das Gehirn steht dabei in Wechselwirkung mit der Umwelt, das bedeutet, dass Lernende neue Informationen unter Einbeziehung bereits vorhandener Informationen verarbeiten und in ein organisiertes Netz vorhandenen Wissens einbinden.

Der Konstruktivismus vertritt den lerntheoretischen Ansatz, dass sich Lernende im Lernprozess eine individuelle Repräsentation der Welt erschaffen und es eine objektive Wahrheit über die Welt nicht gibt. Im Mittelpunkt steht das Konstruieren, Rekonstruieren und Dekonstruieren der Umwelt. Diese Prozesse 
sind unmittelbar an die Handlungen und vorherigen Erfahrungen der Lernenden geknüpft, insofern ist Lernen immer vom Subjekt selbst anhängig. Aus konstruktivistischer Perspektive ist Lernen am effektivsten, wenn die Lernenden ihren Lernprozess umfassend selbst steuern. Allerdings setzt dies eine spezifische Methodenkompetenz voraus. Insofern werden Lernarrangements befürwortet, in denen die Aneignung von Methodenkompetenzen im Mittelpunkt stehen und die Lehrenden nicht nur als Wissensvermittler, sondern eher als Lernprozessberater fungieren. Die Lehrenden sollen sich bei konstruktiven Methoden eher im Hintergrund halten, Lernangebote im Sinne einer Ermöglichungsdidaktik schaffen, Wissensquellen bereitstellen, den Lernprozess beobachten und unterstützen. Vor allem in der Erwachsenen- und Weiterbildungsforschung sind konstruktivistische Modelle nach Arnold und Siebert etabliert (u. a. Arnold, 2005). Nuissl spricht in diesem Zusammenhang von einer „Ubiquität des Konstruktivismusmodells in praktisch allen pädagogischen Zusammenhängen“" (Nuissl, 2006 S. 219).

Aufbauend auf die genannten Hauptströmungen gibt es zahlreiche weitere theoretische Ansätze wie z. B. die Information Processing Theory (Miller, 1956) oder die Cognitive Load Theory (Sweller, 1988), die auf begrenzte Fähigkeiten des Kurzzeitgedächtnisses hinweisen oder die Multimedia Learning Theory (Moreno \& Mayer, 2000), die die Stimulierung unterschiedlicher Sinne durch abwechslungsreiche Lernmethoden hervorhebt. Darüber hinaus gibt es diverse sozial konstruktivistische Ansätze, die das Lernen in situierten und authentischen Kontexten (Lave \& Wenger, 1991) oder auch die Anwendung des Gelernten sowie die Reflexion der Lerninhalte (Schön, 1987) in den Mittelpunkt der didaktischen Konzeption stellen. Ziel eines didaktisch fundierten Lernarrangements ist es, Zugang zu den Lernenden zu schaffen und somit Motivation, Wille und Aufmerksamkeit zum (selbstorganisierten) Lernen zu fördern (Meueler, 1994), welche als Grundvoraussetzungen erfolgreichen Lernens betrachtet werden können.

Moderne, erwachsenenpädagogische Ansätze vertreten die Auffassung, dass situative, explorative und kollaborative Lernmethoden besonders geeignet sind, um einerseits Methodenkompetenzen zu fördern und andererseits formales und informelles Lernen zu verknüpfen (Schön \& Döring, 2011; Specht, Ebner \& Löcker, 2013). Die Ausrichtung erwachsenenpädagogischer, didaktischer Modelle lassen einen deutlichen Wandel hin zu kompetenzorientierten Konzepten erkennen.

Dabei wird betont, dass Kompetenzen nicht vermittelt werden können und sich nur über aktive Handlungen aufbauen lassen. Das erfordert selbstgesteuertes Lernen - und damit ein ganz anderes Lernsetting, als wir es aus üblichen Weiterbildungsveranstaltungen mit lehrendenzentrierten Methoden des Frontalunterrichts kennen. Hierfür wird eine Lernumgebung benötigt, die selbstorganisiertes 
Lernen und Handeln am Arbeitsplatz ermöglicht und die Rolle der Lehrenden von Wissensvermittlern hin zu Lernbegleitern transformiert. Beim Einsatz selbstgesteuerter Lernkonzepte kann darüber hinaus dem relevanten Kriterium der individuellen Passung zwischen Lernstil, Lerntempo, Lerninhalten und Lernmethoden am ehesten entsprochen werden, da die Lernenden den Prozess des Lernens selbst steuern und dieser nicht von außen vorgegeben wird.

Die vorliegende Forschungsarbeit baut überwiegend auf einer konstruktivistischen Lerntheorie auf, wobei auch weitere Ansätze in die theoretische Fundierung einfließen. Das problemorientierte Lernen integriert beispielsweise den kognitivistischen und den situierten (konstruktivistischen) Ansatz, in dem die Instruktionsprozesse des Lehrenden wie auch die Konstruktionsprozesse des Lernenden thematisiert werden (Reinmann-Rothmeier \& Mandl, 1997/2001). Die Balance zwischen Instruktion und Konstruktion in Abhängigkeit von den Lernvoraussetzungen und dem Lerngegenstand stellt entsprechend die zentrale Forderung dar.

Unter Berücksichtigung der eben genannten theoretischen Hauptströmungen und Annahmen wird im Folgenden das Konzept des arbeitsplatzbezogenen Lernens dargestellt und mit Befunden aus der empirischen Lehr- und Lernforschung untermauert. In der Folge werden die extrahierten „Erfolgskriterien“ aus der Lehrund Lernforschung gebündelt und in ein Modell zur Gestaltung intelligenter und hybrider Lernräume überführt (vgl. Abschnitt 3.4).

\subsubsection{Arbeitsplatzbezogenes Lernen}

Um sich dem Begriff des arbeitsplatzbezogenen Lernens systematisch zu nähern, muss man zunächst den Begriff ,Lernen“ an sich beleuchten und in seiner vielschichtigen Bedeutung erschließen. Wissenschaftliche Publikationen zur Lehrund Lernforschung gibt es viele, eine allgemeingültige Definition des Lernens jedoch nicht. Das mag zum Teil darin begründet sein, dass es unterschiedliche Forschungszugänge (Erwachsenenbildung, Pädagogische Psychologie, Soziologie, Neurowissenschaften) gibt, die mit ihren jeweils spezifischen Perspektiven und mit ihren unterschiedlichen Fragestellungen das Lernen Erwachsener erforschen. Dies zeigt sich auch in einer inflationären Verwendung von Begriffen wie „lebenslanges Lernen“, „Lerngesellschaft“, „lernende Organisation“ oder „lernende Region“ (Siebert, 2006). Darüber hinaus existieren etliche verschiedene Lernarten und Begriffsbestimmungen sowie unzählige Abhandlungen zu modifizierten Lerntheorien und Lernansätzen. 
Sofern man die Perspektive auf internationale Literatur erweitert, scheinen noch mehr Lernbegriffe, Lernkombinationen, Bedeutungen und Zusammenhänge zu existieren, die seit 2012 in der Encyclopedia of Sciences of Learning aufgeführt und differenziert beschrieben werden. Diese Enzyklopädie umfasst über 4000 Einträge zu unterschiedlichen Lernformen und Lernbegriffen und beschreibt deren Herkunft, Definition sowie theoretischen Hintergrund. Darüber hinaus verweist die Enzyklopädie auf synonym verwendete Lernbegriffe (Seel, 2012).

Eine systematische Auseinandersetzung mit dem Begriff des arbeitsplatzbezogenen Lernens scheint daher ratsam und notwendig, gerade weil es kaum differenzierte und direkte Anknüpfungspunkte aus der empirischen Lehr- und Lernforschung gibt. Verstärkt wird diese begriffliche Unsicherheit durch die derzeit allseits beliebten ,4.0"-Verwendungen, welche auch im Bildungsbereich (Lernen 4.0, Arbeiten 4.0, Bildung 4.0 etc.) angekommen und beinahe willkürlich gewählt werden, ohne dass die konkrete Bedeutung expliziert würde. Die Fülle an Lernbegriffen führt auch dazu, dass eine Abgrenzung untereinander schwerfällt und die Grenzen gleichsam fließend erscheinen. Die Terminologie der Lehr- und Lernforschung stellt sich insgesamt als höchst divers dar.

Siebert konstatiert entsprechend, dass es sich bei der Lehr- und Lernforschung um ein unübersichtliches Feld handelt (Siebert, 2006). Dennoch soll im weiteren Verlauf des Kapitels der Versuch einer systematischen Dekonstruktion unternommen werden, indem zunächst allgemeine Charakteristika zum Lernen Erwachsener erörtert und diese schließlich mit arbeitsplatzbezogenem Lernen in Beziehung gesetzt werden.

\subsubsection{Befunde aus der Lehr- und Lernforschung}

In seinem Aufsatz zum Lernen Erwachsener, in welchem Ekkehard Nuissl als renommierter Professor für Erwachsenenbildung empirische Befunde aus unterschiedlichen Disziplinen bündelt, verweist er zwar auf unterschiedliche Perspektiven im Forschungsfeld, synthetisiert jedoch sechs Kernbereiche, in dessen Zusammenhängen und Bezügen Lernen grundsätzlich stattfindet (2006):
1. Gedächtnis
2. Inhalt
3. Erfahrung
4. Situation
5. Erfolg
6. Strategien 
Der Autor kann o. a. Kernbereiche des Lernens aus einem systematischen Vergleich unterschiedlicher Definitionen von „Lernen“ aus den Disziplinen Psychologie, Neurowissenschaften, Erwachsenenbildung und Erziehungswissenschaften schlüssig herleiten, wobei er darauf verweist, dass dazu ,belastbare empirische Erkenntnisse von bemerkenswerter Reichweite“ vorliegen (ebenda, S. 219).

Aufbauend auf diesen Kernbereichen lassen sich differenziertere Aussagen zu Prozessen des Lehrens und Lernens zusammenfassen, die auf Basis wissenschaftlicher Erkenntnisse folgende Fragen beantworten:

- Aus welchen Gründen wird gelernt?

- Mittels welcher Aktivitäten wird gelernt?

- Wie wird das Gelernte behalten?

- In welchen Kontexten wird gelernt?

- Welche Inhalte werden gelernt?

- Wann ist Lernen erfolgreich?

Zunächst einmal geht der Autor darauf ein, dass Lernen ein höchst individuelles, biographisches Konstrukt darstellt, das in einer prozesshaften Tätigkeit ausgeführt wird. Als beispielhafte Prozesse führt er Klassifizierung, Kategorisierung, Neugruppierung oder Zugriffsmöglichkeiten auf, die für das Lernen charakteristisch sind. Lernen vollzieht sich demnach auf einer ersten Dimension, die durch interne Prozesse gekennzeichnet ist. Darüber hinaus beschreibt Nuissl eine zweite Dimension, welche durch außen sichtbare Aktivitäten gekennzeichnet ist, die diese inneren Prozesse anregen und steuern.

Demnach ist Lernen nicht als subjektiv isolierter oder isolierbaren Forschungsgegenstand vorfindbar. Lernen ist situiert und geschieht immer in untrennbarer und lebendiger Verflochtenheit mit anderen Tätigkeiten, Situationen und sozialen Konstrukten. Die Erforschung von Anlässen und Bedingungen, von Strukturen und Prozessen, von Wirkungen und Ergebnissen des Lernens in unterschiedlichen lebensweltlichen und institutionellen Kontexten ist grundlegend und konstitutiv für Theorie und Praxis der Erwachsenenbildung.

In diesem Zusammenhang der sozialen Verschränkung von Lernprozessen führen Reinmann-Rothmeier und Mandl (2001) aus der konstruktivistischen Perspektive sechs zentrale Prozessmerkmale für das Lernen auf (2001):

1. Lernen ist ein aktiver Konstruktionsprozess. Wissen kann nur über eine selbstständige und eigenaktive Beteiligung des Lernenden am Lernprozess erworben werden. 
2. Lernen ist ein konstruktiver Prozess. Wissen kann nur erworben und genutzt werden, wenn es in die bereits vorhandenen Wissensstrukturen eingebaut und auf der Basis individueller Erfahrungen interpretiert werden kann.

3. Lernen ist ein emotionaler Prozess. Für den Wissenserwerb ist es zentral, dass die Lernenden während des Lernprozesses positive Emotionen, wie Freude, empfinden. Vor allem Angst und Stress erweisen sich für das Lernen als hinderlich.

4. Lernen ist ein selbstgesteuerter Prozess. Die Auseinandersetzung mit einem Inhaltsbereich erfordert die Kontrolle und Überwachung des eigenen Lernprozesses durch den Lernenden.

5. Lernen ist ein sozialer Prozess. Der Erwerb von Wissen geschieht in der Interaktion mit anderen.

6. Lernen ist ein situativer Prozess. Wissen weist stets situative und kontextuelle Bezüge auf; der Erwerb von Wissen ist an einen spezifischen Kontext oder an eine Situation gebunden. So findet Lernen immer im Rahmen einer bestimmten Lernumgebung statt, die für den Erwerb zentraler Kompetenzen ausschlaggebend ist.

Auf Basis eines situativen, problembasierten Lernansatzes formulieren Mandl, Kopp \& Dvorak (2004) Kriterien für die Gestaltung einer effektiven Lernumgebung. Die vier Designprinzipien für die Gestaltung von Lernumgebungen berücksichtigen das Vorwissen des Lernenden, den Gegenstandbereich, die Bewertung des Lernprozesses und die Einbettung desselben in eine Community:

1. Effektive Lernumgebungen sind wissenszentriert

2. Effektive Lernumgebungen sind lernerzentriert

3. Effektive Lernumgebungen sind bewertungszentriert

4. Effektive Lernumgebungen sind Community-zentriert

Darauf aufbauend formuliert Nuissl (2006) eine dritte Dimension des Lernens, die er aus einer erwachsenenpädagogischen Definition des Lernbegriffes ableitet:

„Lernen ist ein Prozess der relativ dauerhaften Änderung von Verhaltensmöglichkeiten aufgrund von Erfahrungen." (Schrader \& Berzbach, 2005, S. 2-3).

Lernen hat demnach auch eine Konsequenz, es führt zu einem neuen Verhalten bzw. erweitert den Handlungsspielraum. Diese Dimension spiegelt sich insbesondere in den Diskussionen um eine Kompetenzentwicklung der Beschäftigten 
wieder, die über reine Wissensvermittlung hinaus gehen muss (Erpenbeck \& Sauter, 2015).

Konzepte des handlungsbezogenen bzw. arbeitsplatzbezogenen Lernens (,Learning-by-doing“) gehören entwicklungsgeschichtlich sicher zu den ältesten Lehr- und Lernkonzepten überhaupt. In der wissenschaftlichen Diskussion gilt John Dewey (1859-1952) als ein wesentlicher Begründer dieses Ansatzes. Dewey verwendet den Begriff bereits in "Schools of Tomorrow“ (Dewey \& Dewey, 1962), aber auch in „Democracy and Education“ (Dewey, 1916). Lernen muss nach Ansicht von Dewey auf Erfahrung aufgebaut sein. Lernende sollen in einer Lernumwelt experimentieren und dabei selbst die Realität entdecken. Kooperation ist wichtig, nicht nur mit Mitlernenden. Auch dem Lehrer kommt dabei eher die Rolle eines Begleiters als eines Bevormundenden $\mathrm{zu}$, der schon alles weiß. Für Dewey ist der Mensch ein aktives Wesen und ist damit konstruktivistischen Sichtweisen ähnlich. Erkenntnis muss in Handlungsvollzüge eingebettet sein, am besten in der Form eines realen Projekts. Darunter versteht Dewey ein umfangreiches Arbeitsvorhaben, bei dem eine reale Lebensaufgabe von praktischer Bedeutung für das Gemeinschaftsleben bewältigt wird, und zwar so, dass am Ende ein sinnhaft greifbares, praktisch brauchbares Ergebnis steht.

Die Relevanz eines konkreten Handlungsbezugs spiegelt sich auch in den regelmäßig geführten ROI-Debatten von Qualifizierungsmaßnahmen der heutigen Zeit wider, bei welcher die Finanzierung von Weiterbildung auch bestimmte Ziele erfüllen muss. Zur Wirksamkeitsforschung wird abschließend in diesem Unterkapitel näher eingegangen.

Nuissl (2006) komplettiert die Lern-Dimensionen der Prozesse, Aktivitäten und Wirkungen mit der Dimension der Intentionalität. Diese begründet er aus einer erziehungswissenschaftlichen Perspektive heraus. Damit wird dem Lernprozess eine bestimmte Absicht und Zielstellung attribuiert.

Lernen erfolgt nach Nuissl (2006) also auf insgesamt vier Dimensionen mit sechs Kernbereichen, die in einem ersten Schritt der Zuordnung und Strukturierung folgendes Bild zum Lernbegriff zeichnen lassen (Tabelle 2.1):

Tabelle 2.1 Dimensionen und Bereiche des Lernens (nach Nuissl 2006)

\begin{tabular}{|l|l|l|l|}
\hline Prozess & Aktivität & Wirkung & Intention \\
\hline 1. Gedächtnis & 4. Situation & 3. Erfahrung & 5. Erfolg \\
\hline 2. Inhalt & 6. Strategien & & \\
\hline
\end{tabular}


Eine gelingende Lehre ist dabei als eine Optimierung der Balance zwischen Anforderungen und Voraussetzungen der Lernenden zu betrachten, die sich in einer Wechselwirkung oben aufgeführter Dimensionen und Kernbereiche manifestiert. Nuissl (2006) führt diesbezüglich folgende Aspekte auf, die bei einer gelingenden Lehre berücksichtigt werden müssen:

- Klarheit

- Methodenvariation

- Individualisierung

- Motivierung

- Fehlerkultur

- Experimente

Diese allgemeinen Anforderungen lassen sich auf Grundlage der Literaturanalysen noch weiter ausdifferenzieren und zu der von Nuissl (2006) vorgegebenen Struktur hinsichtlich o. g. Dimensionen und Kernbereiche systematisch zuordnen. Im Ergebnis konnten empirisch gesicherte Sachverhalte zu Lernprozessen destilliert und in einen direkten Bezug zu den Anforderungen an die Gestaltung von Lehre in einer Übersicht gebündelt werden. Das Ergebnis ist für jede einzelne Dimension in folgender Tabelle dargestellt.

a) Dimension Prozess (1) und Aktivität (2):

b) Dimension Wirkung (3) und Intention (4):

Trotz dieser Zusammenstellung an Befunden zur Lehr- und Lernforschung, die überwiegend aus wissenschaftlichen Studien aus den Bereichen der pädagogischen Psychologie und Neurowissenschaften entstammen, lässt sich ein Desiderat des aktuellen Forschungsstandes, insbesondere aus Perspektive der Erziehungswissenschaften und Erwachsenenbildung feststellen (Nuissl, 2006). Das zeigt sich u. a. daran, dass in der Regel auf dieselben Leitstudien verwiesen wird, die jedoch aus den 60er und 70er Jahren stammen. Dazu gehört die „Göttinger-Studie“ (Strzelewicz et al. 1966) mit einer soziologisch orientierten Perspektive auf die Bildungsvorstellungen der Bevölkerung, die „Hannover-Studie“ (Siebert \& Gerl, 1975) zu Lehr-Lern-Interaktionen in Erwachsenenbildungskursen und die Heidelberger BUVEP-Studie (Kejcz, 1979), die Lehr-Lernprozesse im Bildungsurlaub und Erwartungen der Teilnehmer*innen zum Gegenstand machte.

Um das zerfaserte Forschungsfeld in seiner Komplexität zu reduzieren und gezielt neue Forschungsprojekte auf vorhandene Lücken zu lenken, wurde im Jahr 2000 das Forschungsmemorandum für die Erwachsenen- und Weiterbildung 
Tabelle 2.2 Valide Befunde zu Lernprozessen und Implikationen für die die Lehre - Dimension Prozess und Aktivität (nach Nuissl 2006)

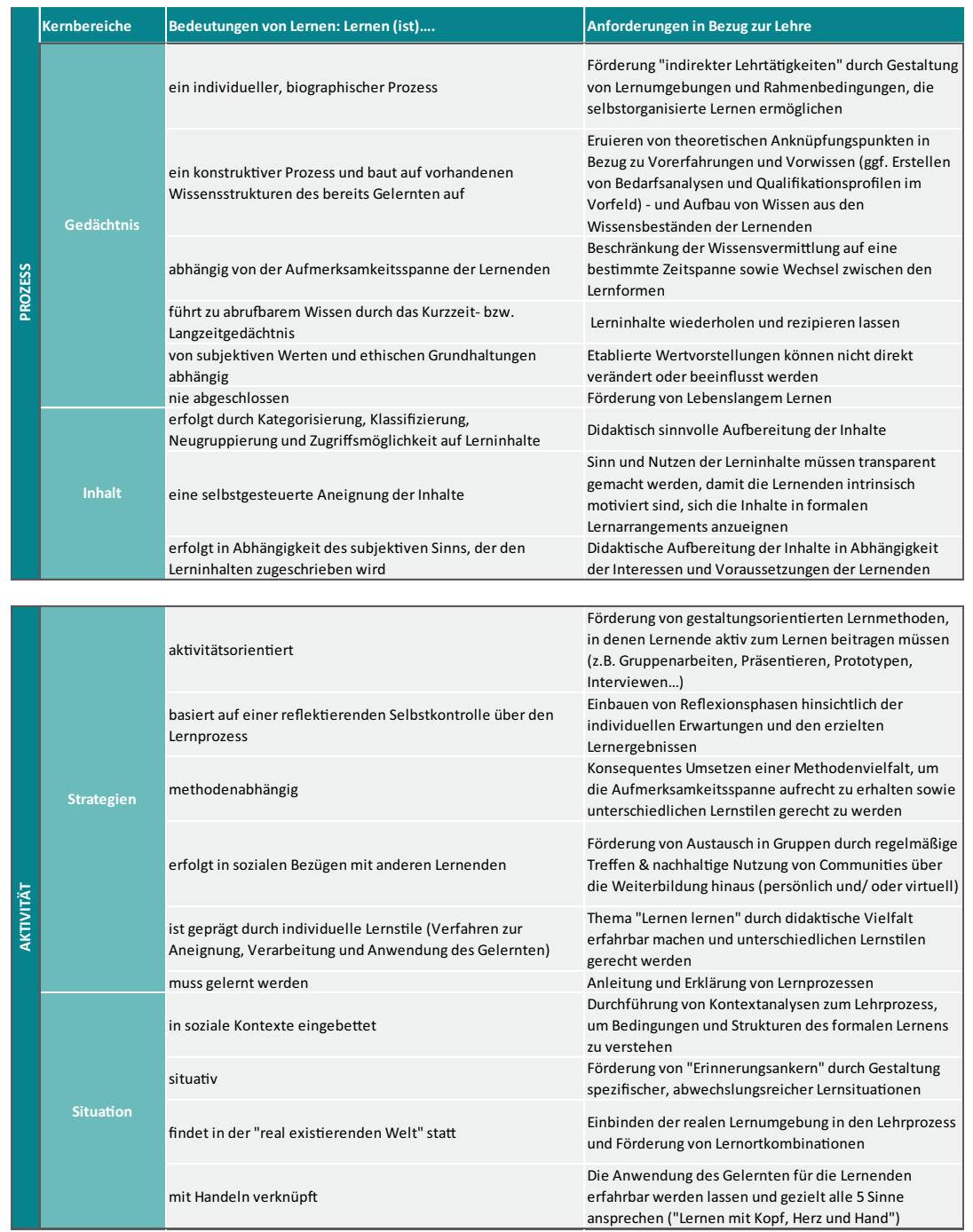


Tabelle 2.3 Valide Befunde zu Lernprozessen und Implikationen für die die Lehre - Dimensionen Wirkung und Intention (nach Nuissl 2006)

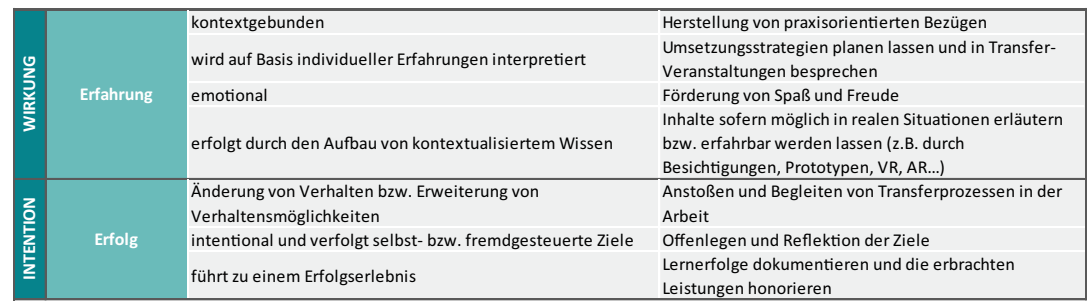

aufgesetzt (Arnold et al., 2000). Das Memorandum wurde im Auftrag der Sektion Erwachsenenbildung der Deutschen Gesellschaft für Erziehungswissenschaft (DGfE) entwickelt. Ziel war es, vor dem Hintergrund des erreichten Forschungsstandes zur Erwachsenenbildung, Schwerpunkte und notwendige Fragestellungen eines zunehmend bedeutsamen Bereiches der Bildungsforschung zu identifizieren, $\mathrm{zu}$ ordnen und $\mathrm{zu}$ benennen.

Eine auf das Memorandum aufbauende Publikation (Zeuner \& Faulstich, 2009), welche eine erwartete Aufarbeitung zum aktuellen Stand der Erwachsenenbildungsforschung leisten sollte, konnte den Ansprüchen nicht gerecht werden. So heißt es in einer Rezension (Nuissl, 2010, S. 92):

„Wenn zwei so profilierte Vertreter der Zunft wie Zeuner und Faulstich ein Buch zu den Resultaten der Erwachsenenbildungsforschung vorlegen [...], dann nimmt man es mit großen Erwartungen in die Hand.“

Bezugnehmend zu den im Memorandum aufgeworfenen Fragestellungen zum Stand der Erwachsenenbildungsforschung und dem darin formulierten Desiderat konstatiert Nuissl (2010, S. 92) in seiner Rezension wie folgt:

\begin{abstract}
„Das vorliegende Buch schafft hier aber keine Abhilfe. Im Grunde handelt es sich um aneinandergereihte Einzelrezensionen empirischer Arbeiten zur Erwachsenenbildung. [...]. Die Arbeiten sowie ihre Ergebnisse sind jedoch einzelnen Untersuchungsfeldern zugeordnet, nicht jedoch systematisiert, gewichtet und zusammenfassend ausgewertet. [...] Feldbezogene Resümees fehlen fast durchweg. [...]. Was bleibt ist ein Materialband, den man nutzen kann, wenn man sich einen ersten Einblick in empirische Arbeiten zur Erwachsenenbildung verschaffen will.“
\end{abstract}

Die Erwachsenenbildungsforschung ist bis heute unübersichtlich und wenig strukturiert, das Schüssler (2012) unter anderem damit begründet, dass eine empirisch 
gesicherte Wirkungsforschung in der Erwachsenenbildung kaum zu leisten ist. Die Autorin bezeichnet dies als eine Paradoxie pädagogischen Handelns, die sich darin manifestiert, dass die Studien je nach Perspektive, Fragestellung und Operationalisierung von ,Lernerfolg“ unterschiedliche Ergebnisse und Zusammenhänge zum Erfolg einer Weiterbildung empirisch nachweisen.

Dies sei darauf zurückzuführen, dass Lernwirkungen und Lernprozesse in keinen Kausalzusammenhängen beschrieben werden können, aufgrund der Vielzahl an Einflussfaktoren. Entsprechend sei eine valide Zuordnung von Intervention und Lernerfolg nicht möglich (vgl. ebenda). Lernprozesse unterliegen einer Vielzahl an kontext-, personen- und prozessspezifischer Faktoren, die in ihren komplexen Wechselbeziehungen untereinander nie eindeutig bestimmbar sind. Darüber hinaus vollzieht sich das Lernen Erwachsener nicht nur auf einer Mikroebene (subjektive Prozesse) der Lernprozesse, sondern wird zudem von Faktoren einer Makroebene (Weiterbildungssystem) beeinflusst.

Obwohl es auf dieser Grundlage keine umfassenden gesicherten Erkenntnisse gibt, verweisen etliche Autoren der Erwachsenenbildung auf Wirkungsannahmen (vgl. Forschungsmemorandum), die sich nur in Teilen durch Erkenntnisse aus der pädagogischen Psychologie und Neurowissenschaften bestätigen lassen.

Reischmann (1993) fasst diese Problematik treffend in folgendem Zitat zusammen:

„Eine enge Wirkungsforschung scheint banal, eine weite nicht leistbar“

Dieser Umstand scheint auch ein Hauptgrund dafür zu sein, dass es insgesamt sehr wenige empirische Studien im Bereich der Wirkungsforschung gibt. Die meisten Befunde im Bereich der Erwachsenenbildung lassen sich einer empirischen Begleitforschung zuordnen.

Ein weiteres Problem ist nach Ludwig (2001), dass die Begriffe „Lernen“ und „Lehren“ oftmals synonym und selbst in der wissenschaftlichen Literatur unscharf verwendet werden. So führt er beispielsweise eine Zielsetzung aus dem Forschungsmemorandum (Arnold et al., 2000) auf, in welcher von ,einer Verbesserung von Lernprozessen“ (S. 10) die Rede ist. Ludwig differenziert an dieser Stelle folgerichtig, dass es sich dabei nur um eine Verbesserung der „Lehrprozesse“ handeln könne, da die Lernprozesse nur von den Lernenden selbst gesteuert werden können. Direkt beeinflussbar sind lediglich Faktoren auf der Makroebene. Subjektive Lernbegründungen wie es Ludwig nennt, könnten allenfalls durch günstige Rahmenbedingungen gefördert und unterstützt werden. Gleichwohl würden aber die organisatorisch-technischen Abläufe auf der Makroebene 
zu relevanten Bedeutungskonstellationen bei den Lernenden führen, so dass es einer ganzheitlichen Betrachtung zwischen Mikro- und Makroebene bedarf.

Ludwig konstatiert in diesem Zusammenhang, dass sich die Erwachsenenbildung überwiegend auf eine Verbesserung der Vermittlungs- und Beratungshandlungen Lehrender bzw. pädagogisch Wirkender fokussiert, sich damit selbst einschränkt und letztlich einseitig forscht. Die unscharfe Verwendung des Lernund des Lehrbegriffs deute überdies darauf hin, dass der Lernhandlung des lernenden Subjekts im Verhältnis zur Lehrhandlung nicht deutlich genug eine eigenständige und differente Handlungslogik zuerkannt würde (vgl. ebenda).

Dementsprechend fordert Ludwig einen differenzierteren Umgang der Begrifflichkeiten, indem zwischen Außenstandpunkt (Lehre) und Subjektstandpunkt (Lernen) unterschieden werden muss. Nicht zuletzt plädieren mittlerweile viele Wissenschaftler (Ludwig, 2008; Nuissl, 2006) auf eine verstärkt interdisziplinär ausgerichtete Forschungsperspektive. Diese würde dazu dienen, ein disziplinäres Profil mit spezifischen Problemstellungen zu entwickeln, um Forschungsprojekte mit anderen Disziplinen entlang der eigenen Forschungsgegenstände „,inter-disziplinieren“ (Röbbecke et al., 2004) zu können. Dies würde aber wahrscheinlich nicht unbedingt zu einer Schärfung, sondern einer Ausweitung des Forschungsfeldes führen.

Siebert (2006, S. 6) nimmt zu dieser Herausforderung in seinem Rückblick zur Lehr- und Lernforschung wie folgt Stellung:

„Die Themen der erwachsenenpädagogischen Forschung weiten sich auf fast alle formalen und informellen Lerngelegenheiten aus. Dies kann als gesellschaftlicher Bedeutungszuwachs dieser Forschung begrüßt, aber auch als Erosion des Forschungsgegenstands bedauert werden.“

Die dargestellten empirischen Befunde zur Lehr- und Lernforschung sollen nunmehr in dem folgenden Abschnitt im Rahmen einer differenzierten Betrachtung zum Lernkonzept „Lernen am Arbeitsplatz“ erweitert werden.

\subsubsection{Begriffsklärung}

Im vorliegenden Abschnitt werden überwiegend theoretische Konstrukte (Annahmen) zum arbeitsplatzbezogenen Lernen vorgestellt und schließlich in den Zusammenhang der empirischen Befunde aus Abschnitt 2.1.2.1 gesetzt.

In der betrieblichen Weiterbildung ist ein Wandel von Lehrveranstaltungen, die „off-the-job“ - sozusagen räumlich und zeitlich losgelöst vom beruflichen Alltag - stattfanden, hin zu arbeitsplatzintegriertem (,on-the-job“) oder arbeitsplatznahem (,near-the-job“) Training zu beobachten. Dieser Wandel von eher 
zentralisierten Lehr-Lern-Formen hin zum Lernen am Arbeitsplatz liegt einerseits in der Diskrepanz von zentralisierten Lehr-Lern-Formen zwischen dem Aus- und Weiterbildungsgeschehen und dem beruflichen Alltag sowie den damit verbundenen Lernmotivationsproblemen begründet. Andererseits wurde erkannt, dass das Lernen im Arbeitsprozess diejenige Lernform ist, die den Erwerb anwendungsbezogenen Wissens und die Entwicklung der Schlüsselqualifikationen Selbststeuerungs- und Kooperationskompetenz am ehesten gewährleistet (vgl. Abschnitt 2.1.2.1). Diese Form gewinnt auch durch den Einsatz neuer Technologien und den damit verbundenen Lernpotenzialen weiter an Bedeutung (vgl. Abschnitt 2.1.3).

Das Lernen am Arbeitsplatz hat sich in den letzten Jahren zunehmend als berufliche Weiterbildungsform etabliert und gewinnt auch im Zusammenhang der Digitalisierung an Bedeutung. Dabei werden informelle und selbstgesteuerte Lernformen zur Entwicklung von anwendungsorientiertem Wissen genutzt. Ziel ist es, am Arbeitsplatz benötigte Fach-, Sozial- und Humankompetenzen auszubilden.

Charakteristisch für das Lernen am Arbeitsplatz ist dabei die fließende Grenze zwischen Lernen und Arbeiten. Dehnbostel (1993) unterscheidet in diesem Kontext zwischen folgenden verschiedenen Modalitäten beim Lernen am Arbeitsplatz:

1. Arbeitsgebundenes Lernen: Lernort und Arbeitsplatz sind identisch

2. Arbeitsverbundenes Lernen: Zwischen Lernort und Arbeitsplatz besteht eine räumliche und arbeitsorganisatorische Verbindung

3. Arbeitsorientiertes Lernen: Lernort und Arbeitsplatz sind räumlich und organisatorisch getrennt

Zum Lernen am Arbeitsplatz werden allgemein Formen von formell-organisierter und informeller Weiterbildung gezählt. Wie auch bei anderen Arten der betrieblichen Weiterbildung wie z. B. den Seminaren/Workshop/Trainings oder dem Selbststudium/Fernunterricht ist das Lernen am Arbeitsplatz ein heterogenes Feld und kann unterschiedliche Formen annehmen. Grundsätzlich können drei verschiedene Lernformen am Arbeitsplatz unterschieden werden (ReinmannRothmeier \& Mandl, 1998):

1. Lernen am Expertenmodell: Bei dieser arbeitsintegrierten Lernform nehmen Vorgesetzte und erfahrene Kollegen einen zentralen Stellenwert ein, da sie für die Lernenden als Expertenmodell fungieren. 
2. Selbstgesteuertes Lernen am Arbeitsplatz: Bei dieser arbeitsintegrierten Lernform zielt der Erwerb von neuen Kenntnissen direkt auf arbeitsplatzspezifische Anforderungen („on Demand“) ab und trägt zu einer konsequenten Verbindung von Lernen und Arbeiten bei. Ein zentraler Punkt beim selbstgesteuerten Lernen am Arbeitsplatz ist, dass trotz der Selbststeuerung der Lernenden nicht auf Anleitung und Unterstützung durch Lehrende verzichtet werden kann. Das ist insbesondere deshalb notwendig, da viele Erwachsene noch nicht zu selbstgesteuertem Lernen bereit sind. Dies belegen z. B. Forschungsergebnisse von Warner, Christie und Choy (1998). Die Ergebnisse dieser und anderer Studien machen auf die Notwendigkeit von instruktionaler Unterstuitzung (bzw. Lernbegleitung am Arbeitsplatz) und sozialer Einbettung des Lernprozesses beim selbstgesteuerten Lernen aufmerksam.

3. Kooperatives Lernen in Kleingruppen: Diese arbeitsintegrierte Lernform hat sich als eine wirksame Form des Arbeitens und Problemlösens bewährt. Vorteile kooperativen Lernens am Arbeitsplatz liegen darin, dass Inhalte aus verschiedenen Perspektiven berücksichtigt werden und das Gelernte flexibel angewendet werden kann. Dadurch können Transferprobleme überwunden werden. Ein effektives Lernen in Gruppen findet aber nur dann statt, wenn Arbeitsaufgaben, Arbeitsorganisation und Gruppenstruktur so gestaltet werden, dass die Kooperation auch tatsächlich notwendig ist und eine positive Interdependenz der Lernenden vorliegt. Um dies zu erreichen, müssen Vorgesetzte ihre Mitarbeiter*innen auf die Möglichkeiten zur Kooperation am Arbeitsplatz aufmerksam machen und deren Notwendigkeit hervorheben. Auch das Vertrauensklima spielt innerhalb des Unternehmens für ein effektives kooperatives Lernen eine entscheidende Rolle.

Zur Unterstützung des Lernens am Arbeitsplatz gibt es im Bereich der Weiterbildung eine Reihe von Lernmethoden, die einerseits das Lernen im Tandem, in Gruppen oder auch in selbstorganisierter Form unterstützen. Zu diesen spezifischen Methoden gehören beispielsweise (Reverse) Mentoring, Coaching, Personal Learning Environment (PLE), Lerninsel, Lernstatt/Forschungswerkstatt, Massiv Open Online Courses (MOOCs), Web Based Trainings (WBTs), Enterprise Social Networks (ESN), Communities of Practice (CoP), Learning Management Systems (LMS), Webinare, Online Video Konferenz, Planspiele, Projektmethode, Qualitätszirkel, Working Out Loud (WOL), Open Space Konferenz etc.. Wichtig ist, dass arbeitsplatzintegrierte Lernformen strategisch und operativ unterstützt werden.

Grundlegende Voraussetzungen für das Lernen am Arbeitsplatz sind folglich ausreichende Spielräume sowie Gelegenheiten und aktive Unterstützungsformate 
für Lernaktivitäten am Arbeitsplatz. Dabei kann festgestellt werden, „dass fachliche, methodische, soziale und personale Kompetenzen in unterschiedlichem Ausmaß von der Ausprägung lernförderlicher Merkmale der konkreten Arbeitsaufgabe und der Unternehmenskultur abhängen“ (BMBF 2001, S. 225). Dieses Ergebnis resultiert aus einem BMBF-Projekt, das im Rahmen des von 1996 bis 2000 laufenden Programms ,Kompetenzentwicklung für den wirtschaftlichen Wandel - Strukturveränderungen betrieblicher Weiterbildung“ durchgeführt wurde (Schiersmann, Iller \& Remmele, 2001).

Konzepte der arbeitsnahen bzw. arbeitsintegrierten Lernformen, die als besonders bedarfsgerecht und flexibel gelten, sind eher gering formalisiert und sind definitorisch durch große Divergenz und mangelnde Abgrenzbarkeit geprägt. Nach einem weiten Verständnis bezeichnen sie Maßnahmen, die ,,in relativer Nähe zum Arbeitsplatz und konkreten Arbeitsgeschehen mit inhaltlich größtmöglichem Praxisbezug“" realisiert werden. Dabei besteht nach wie vor die Schwierigkeit einer eindeutigen Trennung von Arbeiten und Lernen. Dieses Problem, das wohl nur im Einzelfall unter Einbezug der jeweils damit verbundenen Kontexte gelöst werden kann, ist im Hinblick auf arbeitsrechtliche und finanzielle Fragen wie auch auf zeitliche und personelle Ressourcen von hoher Bedeutung, wenn es beispielsweise darum geht, dass Angestellte täglich eine halbe Stunde in Communities of Practice zum fachlichen Austausch aufwenden.

Arbeitsnahe Lernformen werden nicht zuletzt mit der Hoffnung auf Kostenreduzierung in Betrieben implementiert. Im Rahmen von Fallstudien und Modellversuchen wurden etwa Möglichkeiten untersucht, mit denen das Erfahrungswissen der Mitarbeiter*innen allgemein verfügbar und Arbeitsabläufe transparent gemacht werden sollen, wie es z. B. mittels der Erstellung von Lern- und Arbeitsmedien durch Mitarbeiter*innen oder der Selbstevaluierung von Produktionsprozessen geschehen kann. Allerdings ist aus der Praxis bekannt, dass bei Ansteigen des Arbeits- und Zeitdrucks arbeitsnahe Lernformen nur eingeschränkt durchgeführt werden.

Die Autoren Schiersmann et al. (2001) können jedoch trotz der wachsenden Bedeutung des arbeitsplatznahen Lernens keinen Anstieg in Mitarbeiterorientierter bzw. lernförderlicher Arbeitsformen durch Befunde der empirischen Weiterbildungsforschung nachweisen. Anhand der Sonderauswertung der Strukturberichterstattung des Instituts für Arbeit und Technik (IAT) des Wissenschaftszentrums Nordrhein-Westfalen wurde sogar deutlich, dass von 1993 bis 1998 der Anteil der Beschäftigten in partizipativen Arbeitsorganisationen von 39,9\% auf 36,3\% gesunken ist - bei gleichzeitigem Anstieg tayloristischer Arbeitsorganisationen und fremdbestimmter Einzelarbeit von 13,6 \% auf 14,4\% (vgl. ebenda). 
Da diese empirischen Befunde allerdings bereits 16 Jahre alt sind, wären aktuelle Zahlen vor dem Hintergrund der zunehmenden Automatisierung (Stichwort Digitalisierung) sicherlich neu zu interpretieren. Aufgrund mangelnder aktueller, empirischer Befunde zum arbeitsplatzbezogenen Lernen müssen nachfolgend auch theoretische Konzepte und Annahmen diskutiert werden.

Parallel zur gängigen Diskussion um lebenslanges Lernen am Arbeitsplatz erhalten wie bereits dargelegt informelle und selbstgesteuerte Lernformen eine besondere Aufmerksamkeit im erwachsenenpädagogischen Diskurs. Während die traditionellen und in der Regel stark formalisierten Weiterbildungsseminare gerade auch von betrieblichen Vertretern zunehmend als zu teuer und zu wenig praxisrelevant gebrandmarkt werden, werden zunehmend selbstgesteuerte Lernprozesse am Arbeitsplatz forciert, die als informelle Lernformate in Erscheinung treten.

Aus diesem Grund widmet sich der folgende Abschnitt dem informellen und selbstgesteuerten Lernen am Arbeitsplatz. Eine differenzierte Betrachtung von informellem Lernen soll im nächsten Abschnitt nicht unternommen werden, obwohl es auch hier unterschiedliche Ausprägungen, Perspektiven, Begründungsmuster und Begrifflichkeiten gibt. Diese Diffusität legt eine noch zu leistende theoriebezogene Klärung des Begriffs „Selbststeuerung“ nahe, was jedoch den Rahmen der vorliegenden Arbeit sprengen würde. Da das informelle und selbstgesteuerte Lernen allerdings von wesentlicher Bedeutung für ein arbeitsplatzbezogenes Lernen ist, wird im Folgenden Abschnitt der Bezug zum arbeitsplatzbezogenen Lernen und letztlich zu intelligenten und hybriden Lernräumen hergestellt. Grundlegend für den folgenden Abschnitt ist die Diskussion um eine Abkehr von der Lehrerzentrierung hin zur Fokussierung des Lernenden als gestaltendem Subjekt der persönlichen Lernprozesse (vgl. Abschnitt 2.1.2.1).

\subsubsection{Informelles und selbstgesteuertes Lernen am Arbeitsplatz}

Informelles Lernen, gemeinhin als das Gegenteil von formalem Lernen verstanden, ist nichts grundlegend Neues. Aber Entwicklungen wie die Dynamisierung und Digitalisierung der Arbeitswelt, die Unzufriedenheit mit „traditionellen“ Lehr- und Lernarrangements, Veränderungen von Lernkulturen (,von Wissensvermittlern zu Lernbegleitern") und steigende Ansprüche an Transfererfolge, eine abnehmende Bereitschaft zur Investition in Training und Personalentwicklung und die Verfügbarkeit von neuen Technologien zur Unterstützung von Kommunikation und Zusammenarbeit (Social Media) haben dazu beigetragen, dass informelles Lernen ein wichtiges Thema geworden ist. Zudem verspricht man sich durch informelle Lernformen ein Lernen in vernetzten (internen und externen) Strukturen. Eben diese scheinen im Zuge der digitalen Transformation angemessen, 
um durch den Aufbau von Expertennetzwerken mittels Twitter, LinkedIn oder Xing einen hochwertigen Informationskanal zu generieren, der den immer neuen Wissensbedürfnissen am Arbeitsplatz ,on Demand“ gerecht werden kann.

In diesem Zusammenhang wird oft das derzeit sehr beliebte 70:20:10 Modell aufgeführt, das durch den bekannten Corporate-Learning-Berater Charles Jennings bekannt wurde (Singh, 2014).

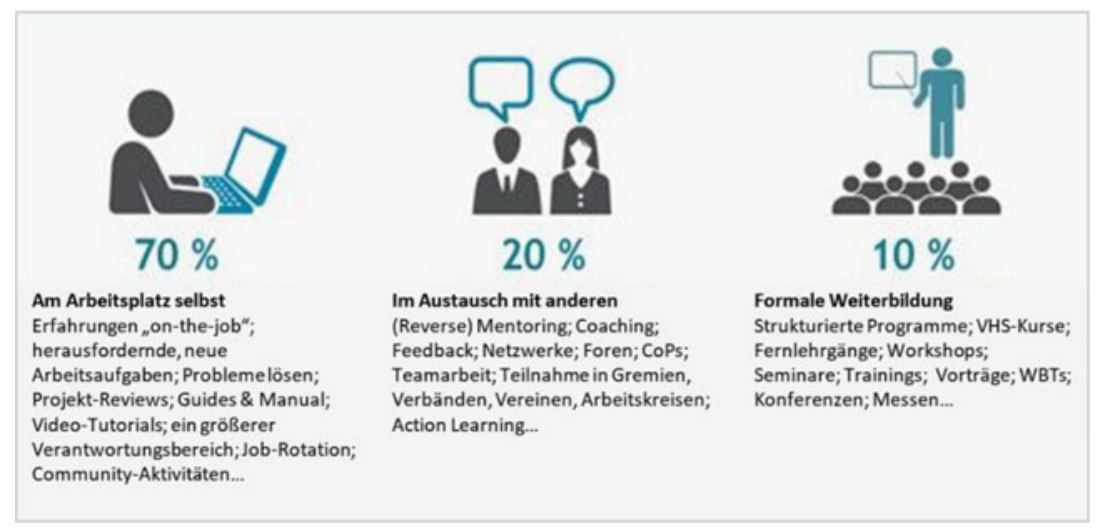

Abbildung 2.1 Das 70:20:10 Modell (Eigene Darstellung nach C. Jennings)

Das Modell geht zurück auf die Autoren Michael M. Lombardo und Robert W. Eichinger, die 1996 in einer Publikation erstmals darüber berichteten, dass das Lernen am Arbeitsplatz einen viel größeren Stellenwert einnimmt als bisher angenommen (Lombardo \& Eichinger, 1996). Durch die konsequente Anwendung im Beratungsalltag konnte Charles Jennings weitere praxisorientierte Erfahrungen sammeln, die er seither auf seinem Weblog veröffentlicht (Jennings, 2017). Wissenschaftliche Befunde bzw. Publikationen, die das Modell validieren, gibt es nicht.

Im Kern geht es bei dem 70:20:10 Modell darum, dass das betriebliche Lernen zum Großteil am Arbeitsplatz im Arbeitsprozess direkt stattfindet. Dabei wurde eine Faustregel entwickelt, wonach $70 \%$ des Lernens informell direkt am Arbeitsplatz stattfindet, $20 \%$ des Lernens über sozialen Austausch erfolgt und lediglich $10 \%$ auf formale Weiterbildungsangebote zurückzuführen ist (vgl. Abbildung 2.1). 
Das 70:20:10 Framework soll dabei unterstützen, informell erlangte Kenntnisse und Fähigkeiten am Arbeitsplatz zu fördern, indem bei der Gestaltung von betrieblichen Lernprozessen insbesondere auch informelle Lernsettings und -formate in die Weiterbildungsarbeit einbezogen werden. Jennings betont dabei, dass es sich bei der zahlenmäßigen Zuordnung um keine absoluten bzw. validen Zuordnungen handelt, sondern dass es sich hierbei um Erfahrungswerte handelt, die er über die eigene Weiterbildungspraxis erlangt hat.

Formen arbeitsintegrierten und informellen Lernens spielen jedoch seit jeher eine große Rolle in der betrieblichen Weiterbildung. Die institutionalisierte Weiterbildung ist nur „das Tüpfelchen auf dem i“ (Dunkel 1976, S. 10). Insofern sind auch aktuelle Publikationen zum Thema im Vergleich zur ,traditionellen, formalen Weiterbildung" nicht unbedingt neu (vgl. Staudt \& Kriegesmann 2000, S. 39, Erpenbeck \& Von Rosenstiel, 2011), liefern aber relevante und insbesondere aktuelle Empfehlungen hinsichtlich einer kompetenzorientierten Weiterbildung im digitalen Zeitalter. So formulieren Erpenbeck \& Sauter (2013) acht konkrete, didaktische Empfehlungen im Kontext eines Blended-Learning Szenarios, welches besonders dazu geeignet ist, formale Weiterbildungseinheiten mit informellen, selbstgesteuerten Lernphasen am Arbeitsplatz zu verschränken:

1. Individuelles, selbstorganisiertes Lernen: Die Lerner organisieren ihre Lernprozesse im Rahmen der vereinbarten Ziele selbstverantwortlich.

2. Organisation und Flankierung durch E-Tutoren bzw. E-Coaches und Trainer*innen: Die Lernbegleiter planen und steuern vor allem die formellen Lernprozesse und unterstützen die Lerner in ihren informellen Lernprozessen. Sie geben den Lernern regelmäßig Feedback und helfen ihnen, ihre Lernprozesse laufend zu optimieren.

3. Problemlösung statt Pauken von Wissen: Der Lernprozess integriert Transferaufgaben und reale Problemstellungen, die die Lerner in ihrer Arbeitswelt zu bewältigen haben.

4. Strukturierungshilfen für individuelles Lernen: Für jede Selbststudienphase werden im jeweils vorhergehenden Seminar verbindliche Vereinbarungen über die Gestaltung der selbstorganisierten Lernphase getroffen.

5. Rückmeldungsstrukturen: Lernen ist dann besonders effizient, wenn die Lerner laufend Rückmeldungen über ihren Lernprozess und ihre Lernleistungen erhalten. Die Rückmeldungen erfolgen grundsätzlich auf zwei Ebenen:

a. Bei standardisierten Aufgaben, z. B. Multiple Choice, Drag and Drop oder Rechenaufgaben, automatisiert über das Lernprogramm. 
b. Offene Aufgaben, z. B. Reflexionen, entscheidungsorientierte Fallaufgaben, Fallstudien oder Transferaufgaben, erlauben keine automatische Bewertung der Lösungen. Es wird deshalb eine Kommunikationsplattform mit E-Portfolio und Learning Community benötigt, die eine entsprechende Kommunikation auch dann zulässt, wenn die Lerner auf verschiedene Orte verteilt sind.

6. Vergleichsmaßstäbe: Die Arbeitsergebnisse anderer Lerner werden netzbasiert zur Verfügung gestellt. Damit kann der Lerner sehen, wie weit er von deren Leistungen entfernt ist. In der Learning Community sowie in Workshops können Arbeitsergebnisse aus der Lerngruppe präsentiert und diskutiert werden.

7. Lernwegflankierung durch Tandems: Diese soziale Flankierung ist eine wesentliche Voraussetzung für erfolgreiche Lernprozesse. Eine besonders bewährte Form ist der Zusammenschluss zweier Lerner zu einem Lerntandem. Hierbei unterstützen sich die Lerner in der Tandemarbeit emotional, motivational und lernstrategisch.

8. Lernwegflankierung durch Kleingruppen: Tandemarbeit reicht nach unseren Erfahrungen im Regelfall nicht aus, um den Lernerfolg im Sinne der Kompetenzentwicklung zu sichern. Notwendig ist eine weitere soziale Flankierung in Kleingruppen, da Gruppen mehr Motivierungsmöglichkeiten und mehr Korrekturmöglichkeiten haben als Einzelpersonen.

Die dargestellten Empfehlungen lassen direkte Bezüge des informellen und selbstgesteuerten Lernens am Arbeitsplatz erkennen, wobei zugleich eine Verschränkung von informellen und formalen Lernformate in Form von Blended-Learning gelingt. Entsprechend gibt es umfassende theoretische Konzepte, auf Basis derer eine ,erfolgsversprechende“ Gestaltungsarbeit aufbauen kann.

Kritik an diesen Konzepten resultieren vielfach über eine nicht strukturierte und systematische Konzeption im Vorfeld. Arbeitsplatzbezogene Weiterbildungsmaßnahmen müssen über den Status eines „learning by doing“ hinausgehen (Wittwer, 2001). Arbeitsplatzbezogenes Lernen verlangt nach entsprechenden Rahmenbedingungen, nach einer Lerninfrastruktur am Arbeitsplatz, die es den Beschäftigten ermöglicht, bewusst und effizient zu lernen (vgl. Abbildung 2.2). Nur auf dieser Grundlage sind Beschäftigte in der Lage, das neuerworbene Wissen bzw. die neuen Fähigkeiten und Fertigkeiten in anderen Situationen anzuwenden (Stichwort Transfer). Die betriebliche Weiterbildung muss hier zusammen mit den Fachabteilungen die Arbeitsplätze arbeitsorganisatorisch und -inhaltlich so gestalten, dass ein Lernen im Arbeitsprozess aktiv unterstützt wird. 


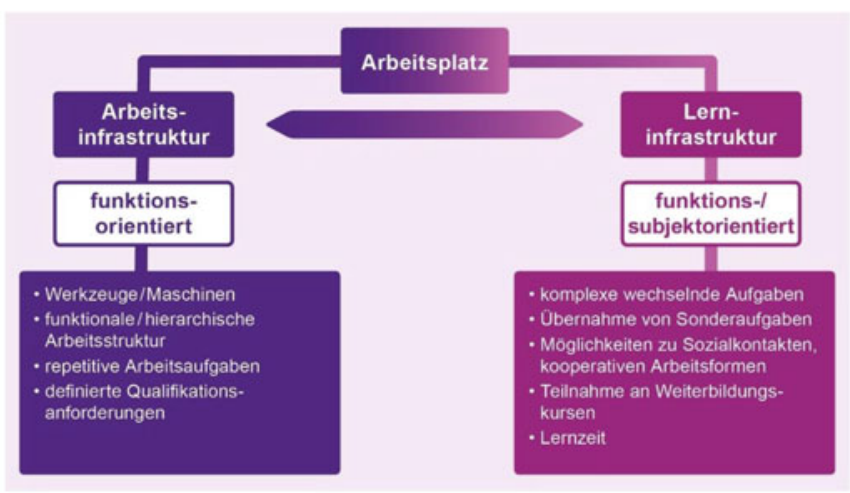

Abbildung 2.2 Arbeitsplatz als Lernplatz (Quelle: Nach Wittwer, 2001)

Auch Straka (2001) verweist auf die Bedeutsamkeit des selbstgesteuerten Lernens von Mitarbeiter*innen und einer direkten Beziehung zu den erlebten Umgebungsbedingungen am Arbeitsplatz. Das in diesem Zusammenhang auftretende Problem, wie in einer nach wie vor fremdbestimmten Arbeitswelt Selbststeuerung von Lernprozessen überhaupt möglich ist bzw. welche Dimensionen dabei erreicht werden können, ist laut Straka noch weitgehend ungeklärt.

Als Unterstützung des selbstgesteuerten Lernens am Arbeitsplatz formulieren die Autoren Sauter \& Sauter (2013) konkrete Lösungsvorschläge, die auf einer Anwendung neuer Medien sowie dem Einsatz moderner Lerntechnologien beruhen. Damit wird es möglich, die selbstgesteuerten Lernprozesse strategisch zu begleiten und aktiv zu fördern. In diesem Kontext beschreiben sie das Konzept einer persönlichen Lernumgebung. Hierbei können die Lernenden sich eine personalisierte Lern-Infrastruktur selbst entwickeln. Persönliche Lernumgebungen (engl.: Personal Learning Environment (PLE)) sind individuelle und cloudbasierte, nach den persönlichen Interessen und Bedürfnissen des Lernenden gestaltete Lernlandschaften, in die sie online Informationen, Erfahrungswissen, Ressourcen oder Kontakte integrieren und Ergebnisse ihrer formalen und informellen Lernprozesse auf der Basis von Standards speichern können.

Aufbauend auf den formulierten Herausforderungen arbeitsplatzbezogenen Lernens wird im Folgenden eine Arbeits- und Lerninfrastruktur vorgestellt, die die 
notwendigen Rahmenbedingungen für selbstgesteuerte Lernprozesse am Arbeitsplatz herstellen kann. Dabei wird das Konzept einer persönlichen Lernumgebung detaillierter erläutert, um eine sich anschließende technologische Weiterentwicklung skizzieren zu können, die sich auf adaptive und ubiquitäre Lernformen bezieht und somit ideale Anknüpfungspunkte für eine mögliche Anwendung des Internet der Dinge auf Lehr- und Lernprozesse liefert.

\subsubsection{Lernen mit persönlichen Lernumgebungen}

Wie in den vorigen Abschnitten dargelegt, bieten digitale Medien vielfältige und leicht nutzbare Ressourcen für selbstgesteuerte und arbeitsplatzbezogene Lernformen. Eine PLE kann dabei als organisierendes Rahmenkonstrukt des selbstgesteuerten, lebenslangen Lernens bezeichnet werden, welche alle zum Lernen (und Arbeiten) benötigten Ressourcen umfasst (Buchem, Attwell \& Torres, 2011). Eine PLE aggregiert entsprechend viele, unterschiedliche und sofern möglich untereinander vernetzte Medien und kann nach individuellen Bedürfnissen zusammengestellt werden.

Durch die rasante informationstechnische Entwicklung stehen viele Möglichkeiten zur technischen Realisierung zur Verfügung. Relativ einfach kann ein individueller "Personal-Desktop" gestaltet werden, der verschiedene, frei zugängliche Applikationen bündelt. Die technische Umsetzung einer PLE ist sozusagen ein Mashup spezifischer Dienste und Tools innerhalb einer Plattform (vgl. ebenda). Eine PLE bündelt verschiedenste Applikationen wie z. B. Social-Media-Kanäle oder auch Email-Accounts, Blogs, Wikis, LMS/LCMS, Feeds, Literaturverwaltungsprogramme, Aufgabenplaner, Cloud-Backup-Systeme, ProjektmanagementTools, Kalender etc., je nachdem, welche Dienste und Tools im Arbeits- bzw. Lernprozess benötigt und als sinnvoll betrachtet werden (Abbildung 2.3).

Das Ziel ist die Entwicklung einer technologischen Infrastruktur, die eine individuelle Kompetenzentwicklung der Lernenden am Arbeitsplatz ermöglicht, indem vorher getrennte Anwendungen bedarfsorientiert zusammengeführt werden.

Eine PLE ist in der Lage, die informellen Lernprozesse zu kanalisieren. In Anlehnung an Sauter \& Sauter (2013) lassen sich charakteristische Merkmale einer zukunftsgerichteten PLE in den folgenden Punkten zusammenfassen:

- die Inhalte und die Lerner-Oberfläche werden vom Lernenden selbst und eigenverantwortlich gestaltet

- der Lernende misst auf Basis der Interpretation seiner Lerndaten und der Ergebnisse aus den Kompetenzmessungen seine Lernfortschritte. Zukünftige 


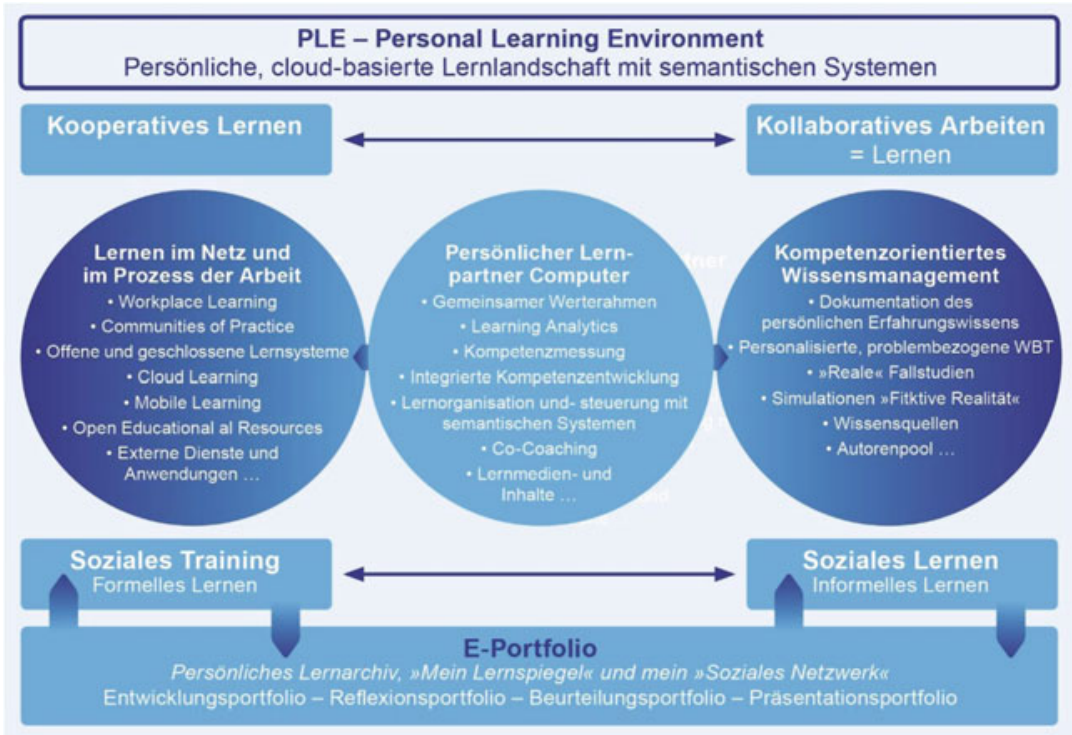

Abbildung 2.3 Struktur einer PLE (Quelle: Nach Sauter \& Sauter 2013)

Leistungen werden vorausberechnet und potenzielle Problembereiche aufdeckt (Learning Analytics)

- die Kompetenzentwicklungsziele der Lernenden werden auf dieser Grundlage selbstorganisiert definiert und die Priorität der Lerninhalte eigenverantwortlich festlegt

- die PLE stellt nach den Interessen und den Kompetenzentwicklungsmöglichkeiten des Lernenden pro aktiv Wissen zur Verfügung

- der Lernende erhält die Möglichkeit, sein eigenes Wissen mit unterschiedlichen Werkzeugen/Tools systematisch aufzubereiten und zu dokumentieren

- der Lernende definiert Zugangsberechtigungen sowie Vernetzungen zu Lernpartnern und sozialen Netzwerken

- der Lernende ist einziger Eigentümer seiner Inhalte

- die persönlichen Daten werden nach den Vorgaben der Lernenden geschützt

Eine PLE bildet damit die notwendige Voraussetzung für selbstorganisiertes und lebenslanges Lernen. Deshalb sollte das System grundsätzlich so gestaltet werden, dass der Lernende seinen persönlichen Lernraum, seine digitale Lernidentität 
„mitnehmen“ oder zumindest löschen kann, falls er sein bisheriges Unternehmen verlassen sollte.

Aus den vorhergehenden Abschnitten lassen sich auf Grundlage der Literaturanalysen differenzierte Anforderungen an die Gestaltung einer arbeitsplatzförderlichen Lernumgebung zusammenfassen. Diese werden im Folgenden in der von Nuissl (2006) vorgegebenen Struktur hinsichtlich Lern- Dimensionen und Kernbereiche systematisch zugeordnet und bauen auf den Tabellen 2.2 und 2.3 (Valide Befunde zu Lernprozessen und Implikationen für die Lehre) auf. Im Ergebnis können konkrete Anforderungen zum arbeitsplatzbezogenen Lernen destilliert werden (Tabelle 2.4 und 2.5).

a) Dimension Prozess (1) und Dimension Aktivität (2):

b) Wirkung (3) und Intention (4):

Da eine PLE eine wichtige Rahmenbedingung für das Lernen am Arbeitsplatz darstellt, soll im folgenden Abschnitt auf automatisierte Verfahren eingegangen werden, die eine zeitsparende und effiziente Art einer PLE-Entwicklung ermöglichen. Unter Berücksichtigung der genannten Merkmale einer Arbeitsund Lerninfrastruktur werden im Folgenden adaptive und ubiquitäre Lernformen vorgestellt, durch welche sich innovative Potentiale durch die Anwendung des Internet der Dinge auf Lehr- und Lernprozesse ableiten lassen.

\subsubsection{Adaptive und ubiquitäre Lernumgebungen am Arbeitsplatz} Adaptives Lernen oder auch „Adaptive Learning“ wurde bereits in den 70-er Jahren des letzten Jahrhunderts entwickelt. Bereits damals existierte die Idee, dass Computer mit Nutzer*innen lernen und ihnen Wissen genau dann zur Verfügung stellen, wenn es benötigt wird. Ein bekanntes und trotzdem nicht erfolgreiches Beispiel war die Büroklammer im Microsoft Office Paket. Die Büroklammer fungierte als virtueller Lernassistent, wurde allerdings kaum genutzt.

Die rapide Weiterentwicklung in unterschiedlichsten Technologiebereichen wie z. B. Internet of Things, Big Data \& Data Sciences, Robotics, HumanMachine-Interaction, User Experience, Artificial Intelligence, Machine Learning, Deep Learning, Reinforcement Learning, Neural Networks, Image Recognition, Semantic, Sensors, Embedded Systems, Microcontrollers, Wearables, RAM, NVIDIA CPU etc. bieten die technologischen Grundlagen zur Gestaltung adaptiver und ubiquitärer Lernszenarien (Brusilovsky \& Peylo 2003; Erpenbeck \& Sauter, 2013 u. 2015; Winkler et al. 2014).

Weber (2012, S. 113) definiert adaptive Lernsysteme wie folgt: 
Tabelle 2.4 Valide Befunde zu Lernprozessen und Implikationen für arbeitsplatzbezogenes Lernen - Dimension Prozess und Aktivität (nach Nuissl 2006)

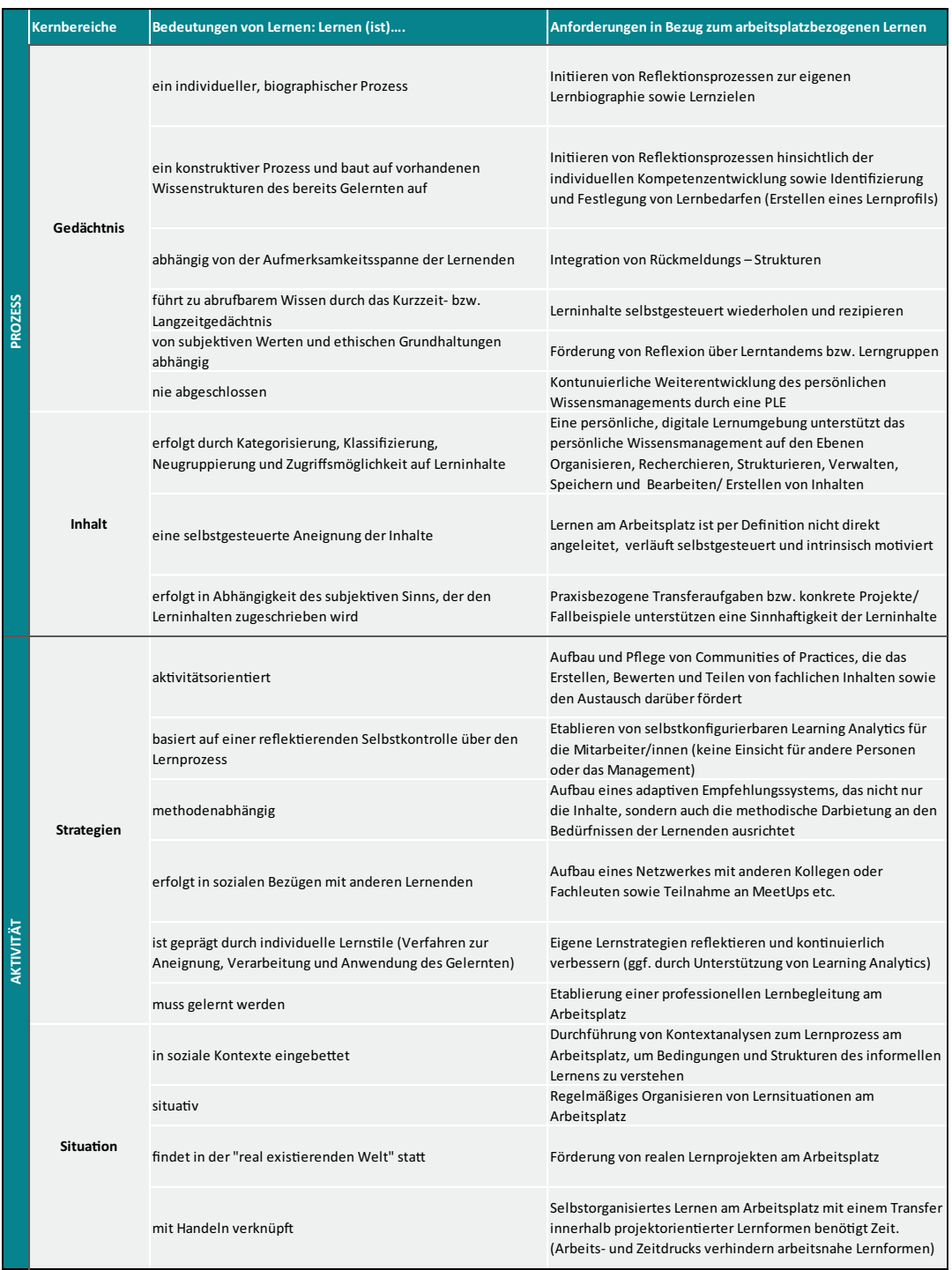


Tabelle 2.5 Valide Befunde zu Lernprozessen und Implikationen für arbeitsplatzbezogenes Lernen - Dimensionen Wirkung und Intention (nach Nuiss1 2006)

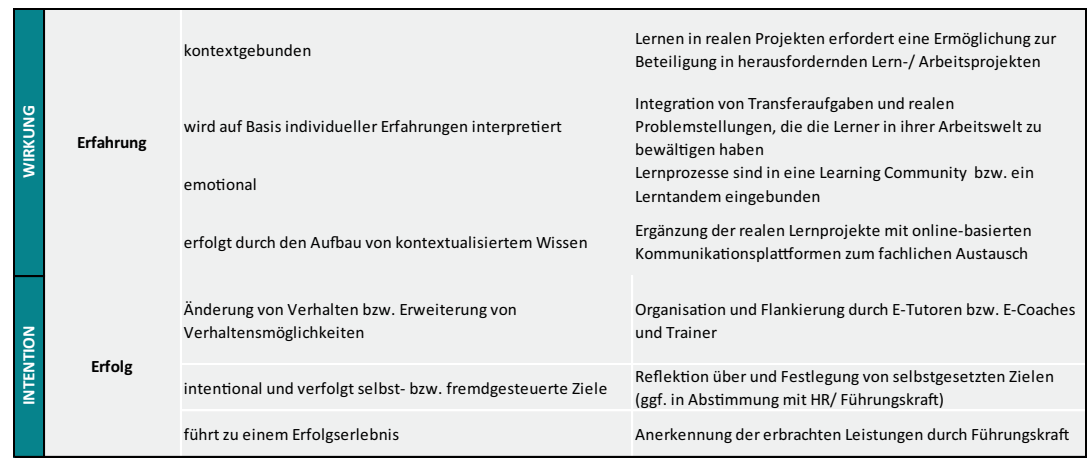

„Adaptive Learning systems (often called Adaptive Learning environments) aim at supporting learners in acquiring knowledge and skills in a particular learning domain. The goal is to enhance the individual learning process with respect to speed, accuracy, quality and quantity of learning. A wide range of different adaptation techniques is used in current Adaptive Learning environments. The application of these techniques is based on information about a particular learner stored in an individual learner model.“

Adaptive Lernformen sind folglich dadurch gekennzeichnet, dass die Lerninhalte den Bedürfnissen der Lernenden entsprechen. Dies wird durch eine komplexe Modellierung von Lernprofilen und einer Auswertung der so generierten Daten (Learning Analytics) ermöglicht. Derartige Systeme sind in den Bereichen Business Intelligence, Social Media Monitoring, Knowledge Management, Data-Driven Marketing, Competitive Intelligence oder auch in der Marktforschung bereits etabliert, im Bildungsbereich stehen derartige Systeme aufgrund mangelnder Wirtschaftlichkeit jedoch noch am Anfang.

Wie bereits erläutert, können adaptive Verfahren mit dem Konzept einer PLE kombiniert werden. Ziel ist es dabei, den Lernenden auf effektive Art und Weise selbstgesteuert zu ermöglichen, auf relevantes Wissen und Informationen zuzugreifen, sofern ein aktueller Lernbedarf (On Demand) am Arbeitsplatz besteht. Adaptive Lernsysteme können hierbei die digitale Lernumgebung (PLE) durch Anwendung von Verfahren aus der künstlichen Intelligenz unterstützen. Die Informationsflut (Big Data) wird hierbei kanalisiert und vorstrukturiert, um Informationsprozesse effizienter zu gestalten (Back 2014; Brusilovsky \& Peylo 2003; Erpenbeck \& Sauter 2015). Es ermöglicht relevante und qualitativ hochwertige 
Informationen automatisiert zu „liefern“, anstatt mühevoll auf unterschiedlichsten Portalen zu recherchieren.

Diese Art von adaptivem Lernen kann in selbstgesteuerten, arbeitsplatzbezogenen Lernprozessen wie auch in formalen Lernszenarien hilfreich sein. Man stelle sich ein formales Lernszenario vor, in welchem in einer Gruppenarbeit eine kurze Recherche zu einem Thema durchgeführt werden soll. Der digitale Assistent identifiziert und aggregiert die relevanten internen wie auch externen Informationen, die dann von den Lernenden bewertet und weiterverarbeitet werden können. Hierfür werden wiederum sinnvolle Werkzeuge (Tools) zur Verfügung gestellt. Die Ergebnisse aus den formalen Lernarrangements können dann kontinuierlich in die individualisierten PLEs eingeflochten werden. Beispielsweise dadurch, dass eine im formalen Lernformat designte Infografik in OneNote (intern) gespeichert oder auf Pinterest (extern) gepinnt oder in Twitter (extern) veröffentlicht wird.

Die Zusammenstellung einer oben skizzierten PLE ist sehr komplex und zeitaufwändig, da die individuellen Qualifikationsprofile zunächst angelegt und die in Frage kommenden Applikationen zunächst einmal recherchiert und auf ihre Tauglichkeit innerhalb der jeweiligen Arbeitsaufgaben geprüft werden müssen.

In diesem Zusammenhang erhalten Verfahren aus der künstlichen Intelligenz wie z. B. Machine Learning eine besondere Bedeutung, wenn es beispielsweise darum geht, passende Tools schneller zu finden, fachliche Expertennetzwerke automatisiert vorzuschlagen oder nützliche Zusatzinformationen z. B. zu passenden internen Videos, externen YouTube-Channels, Veröffentlichungen, Konferenzen etc. zu erhalten. Mittels Auswertung der individuellen Nutzerdaten in Echtzeit (Learning Analytics) kann dem Lernenden der eigene Lernprozess visualisiert und zur Reflektion genutzt werden, was im Hinblick auf selbstgesteuertes Lernen wichtig ist. Parallel dazu können die Daten dazu verwendet werden, um relevante Lernressourcen aufgrund des Lernprofils zu identifizieren und dem Lernenden entsprechende Empfehlungen auszusprechen.

Auf diesen Zusammenhang gehen Buchem et al. (2011, S. 21) wie folgt ein:

\begin{abstract}
„Frequently addressed concepts related to tools include mash-ups based on aggregation of tools and Service Oriented Architectures (SOA) as opposed to closed, monolithic systems. Further aspects include intelligent systems, such as personalised and contextualised recommendation services or expert systems based on semantic technologies and/or artificial intelligence“
\end{abstract}

Ein ähnlicher Ansatz wurde auch im Rahmen des Personal Learning MOOC ${ }^{1}$ von Stephen Downes in 2016 diskutiert, in welchem es um die Entwicklung von

\footnotetext{
${ }^{1}$ https://www.downes.ca/post/65051
} 
digitalen Assistenten ging, die das selbstorganisierte und kollaborative Lernen in PLEs durch intelligente Verfahren unterstützen sollten. George Siemens erörterte diesbezüglich in einem Interview, wie unter Zuhilfenahme von Technologien aus dem Bereich der künstlichen Intelligenz ein Fundament entwickelt werden kann, das als Wegbereiter intelligenter Lernsysteme fungiert (Freigang, 2016) .

Ziel assistierter Lernumgebungen ist es einerseits, passende Empfehlungen zu Inhalten zu liefern sowie geeignete Tools/Werkzeuge entlang des Lernprozesses zum weiteren verarbeiten, reflektieren und speichern anzubieten. Zum anderen geht es darum, das in persönlichen Lernumgebungen organisierte Wissen mit anderen zu teilen und individuelle PLEs in übergreifende Netzwerke einzubetten. Dieser Idee liegt der konnektivistische Ansatz nach George Siemens zugrunde, der den Menschen als vernetztes Individuum betrachtet (Siemens, 2014b). Dieses Netzwerk besteht aus Menschen wie auch aus nicht-menschlichen Quellen, wird durch Social Media erschlossen und fördert auf diese Weise selbstorganisiertes, selbstbestimmtes und kollaboratives Lernen. Aufbauend auf diesen Kernelementen können adaptive Lernformen den Lernprozess auf den folgenden 4 Ebenen der Wissensgenerierung unterstützen:

\section{Relevante Informationen finden:}

Relevante Informationen werden wie oben beschrieben durch semantische Technologien vorstrukturiert und in einen Kontext mit Such- und Filterfunktionen gestellt. Die semantische Wissensbasis wird dabei aus internen sowie externen Datenquellen gespeist (Steuer et al., 2014). Dadurch ist es möglich, Kategorien/Cluster zu definieren, zu welchen die Informationen $\mathrm{zu}$ beziehen sind. Die Kategorien/Cluster sind dabei nicht starr, sondern modifizierbar. Das System ist darüber hinaus lernfähig und schlägt automatisch neue Cluster vor, um ,unknown Unknowns“ zu erschließen. Wichtig ist an dieser Stelle, dass die Informationen aus externen (Internet) sowie internen Datenbeständen (LMS/CMS, Wikis, Intranet etc.) bezogen werden. Die Informationen könnten dann beispielsweise für den Suchbegriff „Internet der Dinge“ in folgende Kategorien vorstrukturiert werden:

- Expert*innen (Newsgroups, Communities-of-Practice, Foren, Blogs etc.)

- Akteure (Unternehmen, Verbände, Vereine, Institute, Forschungseinrichtungen etc.)

- Produkte/Hersteller

- (Model-)Projekte/Best-Practices/Use-Cases

- Konferenzen \& sonstige (Online-)Veranstaltungen (z. B. MOOCs etc.)

- Literatur (Artikel, Fachzeitschriften, Studien, Lehrbücher, Hausarbeiten, Diplomarbeiten, Dissertationen, Habilitationen etc.) 
- Präsentationen (z. B. auf Slideshare)

- Bilder/ Fotos (Gruppiert nach (CC-)Lizenzen)

- Videos (Vimeo, YouTube, fachspezifische Videoportale etc.)

- Social Media (ESN, Facebook, Google+, Twitter etc.)

Die Informationen werden auf dafür vorgesehenen Screens (z. B. Smartboards) visualisiert, teilstrukturiert und von den Lernenden weiterbearbeitet. Eine sich daran anschließende, mobile \& ortsunabhängige Bearbeitung ist selbstverständlich möglich. Dementsprechend können die Lernenden von Ihren Laptops, Smartphones oder Tablets auf die Inhalte zugreifen und diese weiterbearbeiten.

\section{Informationen speichern}

Die Speicherung von relevanten Daten erfolgt automatisch per Backup in cloudbasierten Systemen, so dass auf die Ergebnisse von überall aus zugegriffen werden kann. Am Ende einer Session im Lernraum können zum Beispiel die bisher bearbeiteten Ergebnisse (der jeweiligen Teams) gespeichert und automatisch zu Beginn der nächsten Veranstaltung eingespielt werden. Somit wird ein nahtloses Weiterarbeiten ermöglicht. Falls zwischenzeitlich weitere Änderungen vorgenommen wurden, werden diese angezeigt sowie die dafür verantwortlichen Personen hinterlegt. Die (Such-)Ergebnisse werden nun sukzessive weiter strukturiert und bewertet, beispielsweise kann eine Zuordnung zu ,wichtig“ oder „unwichtig“ erfolgen. Eine automatisch generierte Verschlagwortung kann modifiziert und verfeinert werden, z. B. indem die Ergebnisse nicht nur zu Inhalten, sondern zu bestimmten Personen zugeordnet werden, welche diese dann später weiter ausarbeiten sollen. Bei Bedarf werden geeignete Verschlüsselungsverfahren angewendet, so dass nur dafür vorgesehene Personen sensible Daten abrufen können.

\section{Informationen bearbeiten}

Für die Verarbeitung von Informationen werden automatisch geeignete Werkzeuge zur Erstellung bzw. Weiterverarbeitung von Inhalten angeboten. Die Werkzeuge sind auch hier unterschiedlichen Kategorien zugeordnet (z. B. Dokument, Präsentation, Mind-Map, Infografik, Podcast, Vodcast, Animation, Interaktiver Part, Video, Blog etc.). Für die Erstellung von Dokumenten sind dann z. B. einheitliche Vorlagen je nach Dokumententyp in Word/OpenOffice etc. hinterlegt, zudem ein Literaturverwaltungsprogramm, welches alle bisherigen Zitationen verwaltet. Für eine Präsentation stehen z. B. PowerPoint oder Prezi etc. zur Auswahl sowie eine CC-Bildersuche. 


\section{Informationen präsentieren}

Ein adaptiver und ubiquitärer Lernraum (vgl. Abschnitt 2.3.3) wäre in der Lage, den Tag einer Präsentation zu erkennen und würde zur angegebenen Zeit automatisch in den „Vortragsmodus“ schalten. Ggf. gehen die Jalousien automatisch runter, die Leinwand geht in Position und die Soundanlage wird aktiviert. Zusätzliche Features wie z. B. „Aufnahmemodus“ können aktiviert werden. Dann wird die Präsentation aufgezeichnet, ggf. in andere Büros gestreamt und im Nachgang nochmals bearbeitet (vgl. 3.), um als finales Ergebnis ein Video mit den wichtigsten Informationen im System zu speichern für diejenigen, die z. B. nicht anwesend sein konnten.

Zusammenfassend kann festgestellt werden, dass sich adaptive Lernsysteme ausschließlich auf online-basierte Lernformen beziehen und ihren Ursprung in der Intelligent Tutoring Systems (ITS) Forschung haben. Die Erforschung und Entwicklung ,intelligenter Lernsysteme“ basiert entsprechend auf den Errungenschaften der Künstlicher Intelligenz (engl.: Artificial Intelligence) (Weber, 2012). In diesem Zusammenhang werden häufig Begriffe wie ,intelligenter Tutor“ oder auch ,digitaler Assistent“ verwendet, die darauf hindeuten, dass derartige Lernunterstützung mithilfe von Machine Learning, Neural Networks, Deep Learning etc. betrieben werden. Aufgrund der Komplexität derartiger Anwendungen haben Forschungsprojekte zu ITS allerdings überwiegend den Status eines Pilotprojektes, die unter „Laborbedingungen“ erforscht und entwickelt werden. Außerhalb dieser kontrollierten Strukturen werden ,intelligente Systeme“ bisher kaum eingesetzt, da Kosten und Aufwand noch zu hoch sind (vgl. ebenda). Mit einer kontinuierlichen Weiterentwicklung der oben genannten Technologien ist jedoch in den kommenden Jahren damit zu rechnen, dass adaptive Lernsysteme in ihrer Komplexität und Entwicklungsarbeit günstiger und effizienter werden.

In Ergänzung zu den erläuterten adaptiven Lernformen ermöglicht ubiquitäres Lernen oder auch „Ubiquitous Learning“ eine nahtlose Kombination von virtuellen und physischen Umgebungen und geht über adaptive, online-basierte Lernunterstützung hinaus. Der Begriff hat seinen Ursprung im „Ubiquitous Computing" (vgl. Abschnitt 2.2) und wurde maßgeblich ab 2005 durch die Autoren Bomsdorf (2005), Hwang et al. (2008), Kinshuk \& Graf (2012) sowie Peng et al. (2008) geprägt. Dabei definieren Kinshuk \& Graf den Begriff „Ubiquitous Learning" wie folgt (2012, S. 3361):

„Ubiquitous Learning can be defined as an everyday learning environment that is supported by mobile and embedded computers and wireless networks in our everyday life $[\ldots]$. It is aimed to provide learners with content and interaction anytime and 
anywhere [...]. The learning process includes the real-life experience augmented with virtual information and is adapted to the learner and learner's environment."

Dementsprechend ist der reale Lernkontext, also die Situation und der Ort an dem gelernt wird von großer Bedeutung für ubiquitäres Lernen. Über sogenannte „Embedded Systems“ (vgl. Abschnitt 2.2.1.2) kann die physische Umgebung bzw. der Lernraum mit der virtuellen Welt vernetzt werden, wodurch völlig neue Lernformate innoviert werden können (vgl. Abschnitt 2.3.3). Daraus lässt sich in einem ersten Schritt ableiten, dass ubiquitäre Lernformen besonders geeignet sein könnten, um formales Lernen innerhalb intelligenter \& hybrider Lernräume zu unterstützen sowie mit situativen, informellen Lernerfahrungen (vgl. Abschnitt 2.1.2.1) am Arbeitsplatz zu kombinieren.

Die Autorin Bomsdorf (2005) verwendet in diesem Zusammenhang den Begriff „Ubiquitous Learning Environment“ und beschreibt ubiquitäre Lernszenarien folgendermaßen:

„Ubiquitous Learning is the next step in performing e-learning and by some groups it is expected to lead to an educational paradigm shift, or at least, to new ways of learning. [...] Furthermore, it enables seamless combination of virtual environments and physical spaces."

Auch in dieser Zitation wird deutlich, dass physische Räume bzw. Umgebungen eine relevante Rolle spielen, um ubiquitäres Lernen zu ermöglichen. Ziel ist es, überall und zu jeder Zeit, situations- und kontextbezogen Lernen zu können. Ermöglicht wird dies durch eine ,unsichtbare“ Integration moderner Technologien, die ein „omnipräsentes Lernen“ ermöglichen. Dabei wird die physische Welt mit digitalen Inhalten angereichert und über mobile Endgeräte (wie z. B. Smartphone, Tablet) abgerufen (Kinshuk \& Graf, 2012).

Aus dem vorangegangenen Zitat von Bomsdorf (2005) geht darüber hinaus ein direkter Bezug zum Begriff des ,seamless learning“ hervor. In einer Literaturanalyse von Wong \& Looi (2011) wird kritisch darauf hingewiesen, dass die Begriffe „seamless learning“ und „Ubiquitous Learning“ in der wissenschaftlichen Community teilweise synonym verwendet werden und es einer Konkretisierung bzw. einer Abgrenzung der Begrifflichkeiten bedarf (S. 2370). Dabei umschreiben sie „seamless learning“ wie folgt:

"Seamless learning refers to the seamless integration of the learning experience across various dimensions including formal and informal learning contexts, individual and social learning, and physical world and cyberspace" 
Je nach Perspektive und Vorwissen der Leser*innen können beide Definitionen, die sich einmal auf Ubiquitous Learning (Bomsdorf 2005) und einmal auf seamless learning (Wong \& Looi, 2011) beziehen ähnlich interpretiert werden. Aus diesem Grund differenzieren die Autoren Wong \& Looi seamless learning im Rahmen ihrer Literaturanalyse durch eine Zuordnung von insgesamt 10 Merkmalen, die sie aus der Literatur extrahiert und gegenübergestellt haben. Folgende Merkmale sind demnach ausschlaggebend (frei aus dem Englischen übersetzt):

Seamless learning...

1. umfasst formales und informelles Lernen

2. umfasst personalisiertes und soziales Lernen

3. ist zeitübergreifend

4. ist ortsübergreifend

5. unterstützt einen allgegenwärtigen Zugang zu Wissen

6. umfasst physikalische und digitale Lernwelten

7. kombiniert unterschiedliche Endgeräte (Desktop PC, Interactive Boards, Smartphones etc.)

8. ermöglicht ein nahtloses Wechseln zwischen unterschiedlichen Lernphasen

9. unterstützt die Synthese im Wissensprozess

10. umfasst verschiedene pädagogische Modelle oder Lernaktivitäten

Seamless learning kann dieser Charakterisierung zufolge als ein Teilbereich von „smart learning“ interpretiert werden, da es viele Überschneidungen $\mathrm{zu}$ den von Hwang (2014) definierten Merkmalen (vgl. Tabelle 2.7) gibt, die darüber hinaus im soziotechnischen Ansatz dieser Forschungsarbeit Eingang gefunden haben (vgl. Kapitel 3). Deutlich wird, dass das Konzept des „semaless learning“ die jeweiligen pädagogischen Konzepte mehr ins Zentrum rückt wohingegen dem Ubiquitous Learning, wahrscheinlich auch durch den engen Bezug zum ubiquitous computing, eine eher technikorientierte Perspektive zugrunde liegt.

Demzufolge konstatieren die Autoren Wong \& Looi (2011, S. 2372) folgendermaßen:

"Ubiquitous Learning is more about how ubiquitous technology supports the learners in the right way, in the right place, and at the right time, based on the personal and environmental contexts in the real world (Hwang, et al. 2008). Seamless learning, as defined and described by Kuh (1996) and Chan et al. (2006), is more a learner's habit of mind which may or may not need to be mediated or supported by technology." 
Die vorliegende Arbeit versucht im Rahmen eines holistischen und soziotechnischen Ansatzes, beide Lernformen zu kombinieren.

Bei „Ubiquitous Learning“ werden Technologien genutzt, um die Darbietung der Inhalte in Abhängigkeit der aktuellen Situation und des Lernkontextes variabel anzuzeigen. Darüber hinaus werden die Inhalte aber auch an die Bedürfnisse der Lernenden (adaptives Lernen) angepasst. Ubiquitäres Lernen kann demzufolge als eine kontextgebundene Weiterentwicklung adaptiver Lernsysteme interpretiert werden.

Es geht nicht nur darum, die richtigen Informationen, zum richtigen Zeitpunkt und am richtigen Ort zur Verfügung zu stellen, sondern auch adaptiv zu präsentieren. Das adaptive \& ubiquitäre System ,erlernt“ die Bedürfnisse und bevorzugten Lernmethoden und liefert ,adaptive und kontextsensitive Informationen“. Derartig hoch komplexe Lernansätze erfordern nach Kinshuk \& Graf (2012) die Modellierung vielfältigster persönlicher Faktoren und kontextbezogener Parameter im Vorfeld. Ein ubiquitäres System muss letztlich erkennen, wer der Lernende ist, wo sich der Lernende aufhält, was er dort vorhat und in welchem Kontext er sich dort aufhält. Erst dadurch ist ein ubiquitäres System in der Lage, vergangene Lernpfade abzubilden und mit immer neuen Lernsituationen zu verbinden. Kinshuk \& Graf (ebenda) verweisen auf drei Gestaltungsebenen, die für ubiquitäre Lernformen im Vorfeld zu modellieren sind:

1. The learner model

2. The location model

3. The context model

Die Entwicklung von ubiquitären Lernsystemen ist ein sehr komplexer Vorgang und beruht auf einer Kombination folgender, aufeinander aufbauender Bereiche, die von Bomsdorf (2005) detailliert beschrieben wurden:

1. Individuelles Profiling (Qualifikationen, Lernziele, Interessen, bevorzugte Lernmethoden etc.)

2. Zugang zu Meta Daten (Auswertung von Big Data aus internen/externen Datenbeständen)

3. Set an Lernressourcen (Werkzeuge, Lerngegenstände, Tools, Dienste, Kalender, Mail etc.)

Das bedeutet, dass im Vorfeld zunächst eine umfassende Bedarfsanalyse der Lernenden durchzuführen ist. Darauf aufbauend werden interne und externe Datenbestände identifiziert, die im Zuge der Lernprozesse relevante Inhalte liefern 
und an das Lernsystem angebunden werden können. Dazu gehören beispielsweise auch Netzwerke. In einem dritten Schritt werden dann konkrete Lernressourcen identifiziert und bereitgestellt, die individuelle Lernprozesse unterstützen können.

In Ergänzung dazu führt Hwang (2014) weitere Bereiche auf, die für ubiquitäre Lernsysteme analysiert, geplant und implementiert werden müssen:

4. Zustand der Lernenden (Aufenthaltsort, Temperatur, Zeit etc.)

5. Lernkontrolle (Was wurde gelernt? Wo gab es Probleme? Wurden Lernziele erreicht?)

6. Hilfe \& Feedback (z. B. Leitfänden, Feedback-Systeme etc.)

Die differenzierte Literaturanalyse zu den Lernbegriffen ,,adaptives und ubiquitäres Lernen“ hat unter Reflektion zum Begriff des „seamless learnings“ Unschärfen zu Tage gefördert, die eine stringente, eindeutige Begriffsbestimmung in der vorliegenden Forschungsarbeit nicht zulassen. Die Grenzen zwischen adaptivem und ubiquitären Lernen sind fließend, wobei sich selbst die Expert*innen nicht immer einig sind und sich teilweise sogar widersprechen. Besonders deutlich wird dies in einer Übersicht von Hwang (2014), wobei er charakteristische Merkmale adaptiven und ubiquitären Lernens verglichen und gegenübergestellt hat (vgl. Tabelle 2.6 - frei aus dem Englischen übersetzt):

Trotz der ermittelten Unschärfen ist es wichtig, diese modernen Lernansätze weiterzuentwickeln. Die Datenmenge wächst und potenziert sich stetig. Für Wissensarbeiter*innen bedeutet dies sprichwörtlich, die Nadel im Heuhaufen zu finden. Das Problem ist der Datenmangel trotz Datenflut. Dies ist eine von vielen Herausforderungen, mit denen die Gesellschaft im Zuge der Transformation zur Wissensgesellschaft umgehen muss, nämlich diese verteilten, unstrukturierten Daten zur Wissensgenerierung handhabbar $\mathrm{zu}$ machen, indem geeignete Rahmenbedingungen für ein effektives, informelles wie auch formales Lernen am Arbeitsplatz entwickelt werden.

Durch die Nutzung modernster Technologien innerhalb von adaptiven und ubiquitären Lehr- und Lernprozessen verschwimmen zunehmend die Grenzen zwischen unterschiedlichen Lernformen und Lernmethoden. Es entstehen Mischformen des Lernens, die zwischen formalen und informellen Lernsettings, zwischen selbstorganisiertem und sozialen Lernen, zwischen verschiedenen Lernzeiten und Lernorten sowie zwischen analogen und digitalen Lernformaten $\mathrm{zu}$ verorten sind (Specht et al., 2013). Derartige Lernszenarien ermöglichen unter Verwendung modernster, mobiler, drahtloser Informationstechnologien wie dem Internet der Dinge ein fließendes (hybrides) Zusammenwirken zwischen physischen Lernräumen und einer digitalen Lernumgebung. 
Tabelle 2.6 Vergleich zwischen ubiquitärem und adaptivem Lernen (aus dem Englischen nach Hwang 2014)

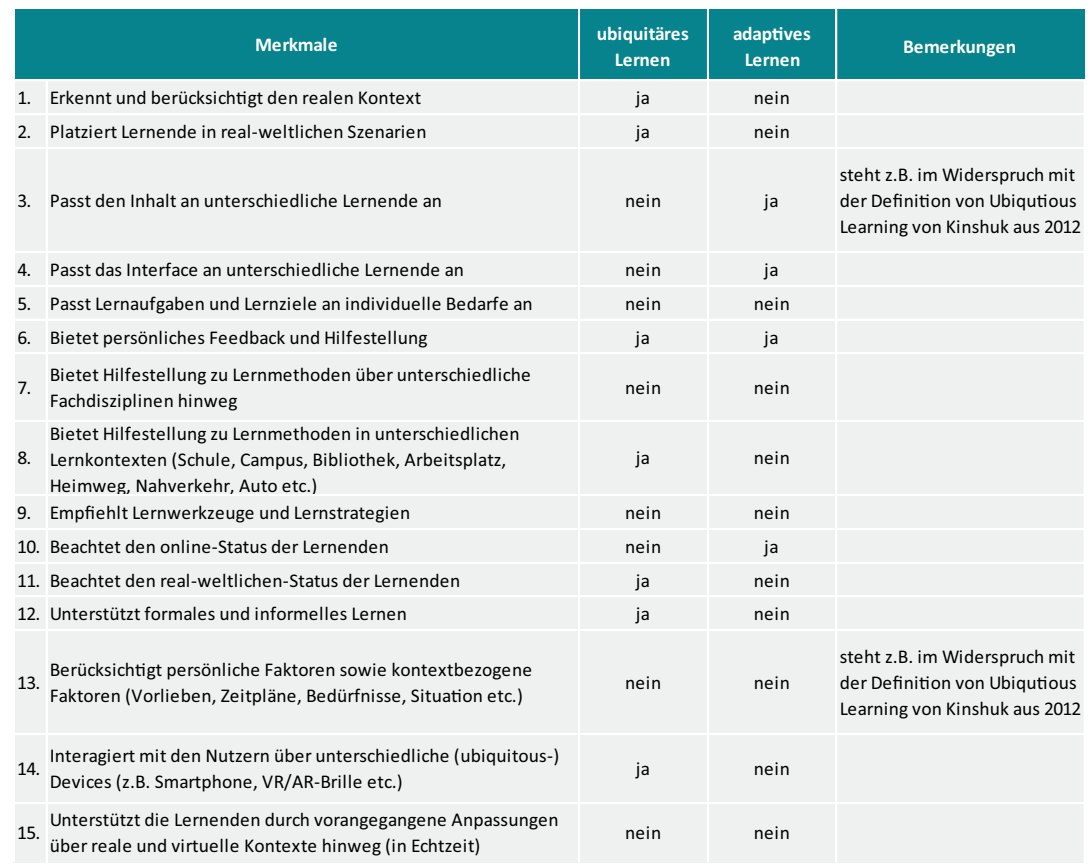

\subsubsection{Schlussfolgerungen}

Lernen ist wie im vorhergehenden Abschnitt empirisch dargelegt, von den Prozessen, Aktivitäten, Wirkungen und Intentionen auf der Mikroebene der Subjekte abhängig und steht darüber hinaus auf der Makroebene in Wechselwirkung mit organisationalen Strukturen der formalen Weiterbildung. Im Rahmen des arbeitsplatzbezogenen Lernens zeigen sich insbesondere Verflechtungen zur täglichen Tätigkeit, also zu den jeweiligen Aufgaben und Kompetenzprofilen der entsprechenden Berufsbezeichnung in einer Organisation.

Moderne Konzepte des „Workplace Learning“ zielen im Zuge der digitalen Transformation auf eine integrierte Kompetenzentwicklung im Prozess der Arbeit selbst sowie auf eine Unterstützung von vernetzten und sozialen Lernformen. Diesem Ansatz folgend ist Lernen vor allem vor dem Hintergrund einer selbstorganisierten Kompetenzentwicklung zu verstehen und findet mehr und mehr in 
und mit dem Netz statt. Das Internet ist dabei einer der wichtigsten Zugänge innerhalb moderner Kompetenzentwicklungsansätze. Die Ausrichtung erwachsenenpädagogischer, didaktischer Modelle lassen einen deutlichen Wandel hin zu kompetenzorientierten Konzepten erkennen, die den Lernenden selbst ins Zentrum rücken. Dies impliziert eine Abkehr von den traditionell eher lehrendenzentrierten Lernformaten hin zu offenen und selbstgesteuerten Umgebungen. Die (passive) Nutzung eines Lernmanagement-Systems (LMS) wird zu einer (aktiv) gestalteten persönlichen Lernumgebung. Insbesondere die digitalen Medien bieten für selbstgesteuerte und arbeitsplatzbezogene Lernformen vielfältige und leicht nutzbare Ressourcen.

Nach Kerres (2016) braucht es im Rahmen der Digitalisierung und der damit verbundenen hoch komplexen Bildungsarbeit sowohl einen Kulturwandel als auch ein systematisches Veränderungsmanagement. Mit der Digitalisierung der Bildung wird ein Veränderungsprozess angezeigt, der über ein bisheriges E-Learning Verständnis hinausgeht und den gesamten Bildungsprozess durchdringt. Die Chancen und Möglichkeiten der digitalen Technologien eröffnen sich in besonderer Weise, wenn sich der Blick auf eine ganzheitliche Bildungsarbeit im Ganzen fokussiert und insbesondere die personalisierten Lernprozesse in den Vordergrund rücken. Die Digitalisierung der Bildung ist pervasiv, sie durchdringt alle Prozesse, Orte und Formate der Bildungsarbeit (vgl. ebenda). Für die vorliegende Forschungsarbeit lässt sich demnach schlussfolgern, dass im Rahmen der gestaltungsorientierten Bildungsforschung eine ganzheitliche Perspektive eingenommen werden muss, die subjektive, soziale, interaktive, kulturelle sowie technische Faktoren gleichermaßen berücksichtigen muss.

Insofern ist eine einseitige Förderung von z. B. informellem Lernen isoliert angewendet keine zufriedenstellende Lösung. Wenn es beispielsweise darum geht, Produktschulungen in der Fläche durchzuführen, Anwender*innen zu neuen IT-Infrastrukturen zu schulen oder Führungsprinzipien zu verankern, gibt es zu didaktisch sauber aufgebauten digitalen Schulungsmedien (WBTs) keine Alternative. Wie das „Swiss Center for Innovations in Learning“ (SCIL) in seinem Arbeitsbericht 24 von Januar 2013 sehr treffend beschrieben hat, geht es weniger um ein entweder/oder, sondern vielmehr um ein Kontinuum (Seufert et al., 2013). Wo das informelle, adaptive oder auch ubiquitäre Lernen anfängt und wo es aufhört, ist eine Sache der jeweiligen Definitionen, die wie im vorhergehenden Abschnitt hergeleitet wurde nicht eindeutig zu dekonstruieren bzw. zu spezifizieren sind. Wichtig ist es daher, die jeweiligen Formate didaktisch sinnvoll miteinander zu verzahnen. Genau an diesem Punkt setzen die hoch modernen Technologien wie das Internet der Dinge ein (vgl. Abschnitt 2.2), die 
die vorhandenen Brüche zwischen formalen Lernsettings in klassischen Schulungsräumen mit informellen Lernformen innerhalb intelligenter und hybrider Lernräume auflösen können (vgl. Abschnitt 2.3). Digitalisierte Bildungsformate wie adaptive und ubiquitäre Lernsysteme erfordern eine komplexe Gestaltungsund Modellierungsarbeit, die über mehrere Ebenen und Parameter vollzogen werden muss.

Im Kern geht es letztlich immer darum, zunächst die Dimensionen des Lernens (Prozesse, Tätigkeiten, Wirkungen und Intentionen) im Vorfeld zu verstehen und genau zu analysieren, um darauf aufbauende organisationale Rahmenbedingungen und Unterstützungsformate zu innovieren. Eine zunehmende Verknüpfung von betrieblicher Weiterbildung mit Personal- und Organisationsentwicklung macht es darüber hinaus nötig, entsprechend vielschichtige Frameworks so anzulegen, dass die Wechselwirkungen der Einflussbereiche stärker in den Blick genommen werden.

In Anbetracht der dargelegten Befunde sowie erwachsenenpädagogischer Diskurse lässt sich Folgendes für vorliegende Forschungsarbeit ableiten:

Aus bildungswissenschaftlicher Perspektive verfolgt die Forschungsarbeit einen ganzheitlichen, gestaltungsorientierten und interdisziplinären Zugang. Das bedeutet, dass die aus der empirischen Weiterbildungsforschung vorliegenden Befunde und Faktoren einer „gelingenden“ Lehre als Rahmung der Arbeit zwar berücksichtigt, nicht aber in validen Kausalzusammenhängen dargestellt werden. Feststehende, kausale Zusammenhänge zu beschreiben, würde das Ziel der Arbeit verfehlen, da letztlich ein Transfer der Erkenntnisse im Mittelpunkt steht und jede Organisation unterschiedliche Ausgangspositionen und Rahmenbedingungen mit zudem variierenden Schwerpunkten aufweisen würde.

Darüber hinaus wird explizit zwischen informellen Lernprozessen am Arbeitsplatz und formalen Lernprozessen in intelligenten und hybriden Lernräumen unterschieden mit dem Ziel, die Brüche zwischen diesen Lernformen zu minimieren.

In diesem Kontext wird in den kommenden Kapiteln der Versuch unternommen, die allzu oft isoliert betrachteten Mikroprozesse der Lernenden in einer Verschränkung mit den Makroprozessen auf organisationaler Ebene zu erweitern. Hierbei werden aktuelle Erkenntnisse aus der Lehr- und Lernforschung gebündelt und in einem ganzheitlichen, soziotechnischen Gesamtsystem betrachtet (vgl. Abschnitt 3.4). 


\subsection{Das Internet der Dinge}

Im folgenden Abschnitt werden unterschiedliche Definitionen des Begriffes „Internet der Dinge“ (engl.: Internet of Things) zusammengetragen sowie Unterschiede und Gemeinsamkeiten herausgearbeitet. Ziel ist es, die Bedeutung sowie die Besonderheiten des Internet of Things (IoT) systematisch zu analysieren und als theoretische Basis der vorliegenden Arbeit darzulegen.

Die systematische Literaturrecherche wurde im August 2015 durchgeführt, wobei das Vorgehen folgende Arbeitsschritte umfasste:

- Festlegung der primären Suchbegriffe auf „Internet der Dinge“ und „Internet of Things"

- Recherche über das Internet, Google Scholar sowie über den ZIH WebVPN Service der TU Dresden, um auf alle Publikationen der Sächsischen Landesbibliothek - Staats- und Universitätsbibliothek Dresden (SLUB) zugreifen zu können (inkl. Fachdatenbanken)

- Identifizierung von 83 relevanten Quellen

- Speicherung und Verschlagwortung der Fundstellen in einem Literaturverwaltungsprogramm (Zotero)

- Sichtung und Strukturierung der Quellen mit Hilfe eines systematischen Textanalyseverfahrens. Dabei wurde die SQ3R-Methode nach Robinson (1978) angewendet (vgl. Abbildung 3.24).

- Inhaltsanalytische Auswertung von 26 Schlüsselwerken zum „Internet der Dinge“" (überwiegend aus dem deutschsprachigen Raum)

- Die systematische Suche mit dem Keyword „Internet der Dinge“ wurde mit einem Schneeballverfahren kombiniert, d. h., dass die Suche über das Literaturverzeichnis einschlägiger Publikationen erweitert wurde. Besonders häufig zitierte Literatur wurde entsprechend den Schlüsselwerken zugeordnet.

\subsubsection{Begriffsklärung und Merkmale}

Eine fundierte Dekonstruktion und Systematisierung der Bedeutung des Internet der Dinge durch einen Vergleich unterschiedlicher Begriffsdefinitionen stellt die Basis zur Generierung wissenschaftlicher Erkenntnisse dar. Von daher wird im Folgenden der Begriff „Internet der Dinge“ bzw. „Internet of Things“ (IoT) erörtert und in seiner Bedeutung erläutert.

Grundlage dieser Analyse ist die Auswertung von 26 Quellen unter dem Aspekt der Definition und Beschreibung des Begriffes „Internet der Dinge“ bzw. 
„Internet of Things“ (IoT). Die verwendeten Quellen stammen aus den Bereichen Politik (z. B. Europäische Kommission, BMWi), Wissenschaft und Forschung (z. B. Fraunhofer, Universität St. Gallen) und Wirtschaft (z. B. Cisco, IBM, Siemens). Eine Übersicht der Kernaussagen ist auf dem Portal Slideshare ${ }^{2} \mathrm{zu}$ finden.

Der Begriff „Internet der Dinge“ geht zurück auf das Auto-ID Center am Massachusetts Institute of Technology (MIT), das im Jahr 1999 erstmalig eine firmenübergreifende RFID-Infrastruktur entwarf. Kevin Ashton, der Mitgründer und damalige Leiter des Auto-ID Center, verwendete in einem Vortrag als Erster die Formulierung ,Internet of Things“ (Fraunhofer IML, 2015).

„I could be wrong, but I'm fairly sure the phrase „Internet of Things“" started life as the title of a presentation I made at Procter \& Gamble $(\mathrm{P} \& \mathrm{G})$ in 1999.“

(Ashton, 2009)

Nach Ashtons Vision sollten Computer fähig sein, unabhängig vom Menschen Informationen zu beschaffen. Computer müssten die reale Welt begreifen - ohne von Menschen bedient zu werden. Sodann würden viele Verluste und Kosten erspart bleiben (Fraunhofer, 2015).

\subsubsection{Internet der Dinge und Smart Objects}

Ein zentrales Merkmal des Internet der Dinge, das sich bereits aus dem Begriff selbst ableiten lässt, sind die sogenannten „Smart Objects“ - also ,intelligente Dinge“. Diese entstehen dadurch, dass Alltagsgegenstände (Objekte/Dinge) mit „technischer Intelligenz" angereichert werden. Die Autoren Fleisch und Thiesse (2014) beschreiben diese Besonderheit folgendermaßen:

„Das Internet der Dinge bezeichnet die Idee eines erweiterten Internet, welches neben klassischen Rechnern und mobilen Endgeräten auch beliebige physische Gegenstände mittels Sensoren und Aktuatoren in seine Infrastruktur einbindet und so zu Anbietern bzw. Konsumenten verschiedenster digitaler Dienste macht.“

Durch das Internet der Dinge wird die physische Welt der Gegenstände mit der virtuellen Welt der Daten verbunden. Das Internet der Dinge führt letztlich zu einer vollständigen Fusion, zu einer Hybridisierung zwischen der analogen und digitalen Welt. Die Grenzen verschwimmen und sind nicht mehr klar voneinander

\footnotetext{
${ }^{2}$ https://de.slideshare.net/Alonvy/was-ist-das-internet-der-dinge
} 
zu trennen. Smarte Objekte/Dinge sind in der Lage, ihre Umgebung wahrzunehmen und Informationen zu verarbeiten. Diese „technische Intelligenz“ kann sich dabei sehr stark unterscheiden und reicht von einer eher passiven Informationsaufnahme, -speicherung und -verarbeitung bis zur autonomen Durchführung von Aktionen, indem sie auf ihre Umgebung reagieren und mit ihren Nutzer*innen innerhalb eines Embedded Systems (vgl. Abschnitt 2.2.1.2) interagieren (Botthof \& Bovenschulte, 2009). Technologische Grundlagen sind hier insbesondere RFID, Sensorik und Aktuatorik.

Die Idee eines Internets der Dinge hat seine Ursprünge in den Konzepten des Anfang der 90er Jahre von Mark Weiser skizzierten „Ubiquitous Computing“ (Weiser, 1991), welche in den Folgejahren von anderen Forschern aufgegriffen und weiterentwickelt wurden. Grundgedanke des Ubiquitous Computing ist ebenso wie der nahezu deckungsgleichen Begriffe „Pervasive Computing“ (Lucke \& Rensing, 2014; Estrin et al. 2002) oder „Ambient Intelligence“ (Aarts \& Harwig, 2002) - eine Erweiterung beliebiger physischer Gegenstände über ihre bestehende Form und Funktion hinaus durch mikroelektronische Komponenten. Die so entstehenden ,smarten“ Gegenstände bilden, mit digital verknüpfter Prozesslogik, Sensorik und der Möglichkeit zur Vernetzung ausgestattet, ein Internet der Dinge, in dem der Computer als Gerät verschwindet und in den „smart Objects“ der physischen Welt aufgeht (Mattern, 2005).

Zusammenfassend kann man Smart Objects als allgegenwärtig, unsichtbar und autonom bezeichnen (Gabriel et al. 2010), welchen folgende charakteristische Eigenschaften zugrunde liegen (Fleisch \& Thiesse 2014):

- Identifikation

- Kommunikation

- Sensorik

- Aktorik

- Speicher

- Benutzerschnittstelle

Ermöglicht und forciert wird das Internet der Dinge durch technologische Trends, die die Autoren Botthof und Bovenschulte (2009) wie folgt zusammenfassen:

- Miniaturisierung/Systemintegration

- Konvergenz

- Dezentralisierung

- Vernetzung

- Selbstorganisation 
Die Autoren Mattern und Flörkemeier (2010) führen in diesem Zusammenhang auf, dass die „Vision“ des Internet der Dinge über oben genannte Charakteristika weitere Grundlagen zu erfüllen hat, die in den Bereichen Effizienz, Skalierbarkeit, Verlässlichkeit, Sicherheit und Vertrauenswürdigkeit zu verorten sind. Als weitere Herausforderungen (vgl. auch Abschnitt 2.2.1.7) formulieren die Autoren die Einhaltung gesellschaftlicher und politischer Erwartungen, die darüber hinaus in Einklang mit ökonomischen Interessen gebracht werden müssen.

Schlussfolgernd kann festgestellt werden, dass sich eine erste Besonderheit des Internet der Dinge auf die Digitalisierung von alltäglichen Dingen bezieht, die bisher nicht mit dem Internet verbunden waren bzw. über keine elektrischen und informationsverarbeitenden Komponenten verfügt haben. Die Auswirkungen sind dabei enorm, weil dies letztlich jeden Gegenstand in unserem Alltag betreffen könnte.

Ein gutes und bereits weit verbreitetes Beispiel liefern Bücher. Einige Kinderbücher sind heutzutage bereits mit Sensoren und Aktuatoren ausgestattet und geben per Tastendruck Musik, Tiergeräusche oder auch auditive Zusatzinformationen zu bestimmten Inhalten wider. Komplexere Lerneinheiten werden durch einen zusätzlichen „Smart-Pen“ ermöglicht. Über diesen können immer neue Module über das Internet heruntergeladen werden. Letztlich können auch Spielkarten $^{3}$, Spielfiguren, ein Globus, ein Vulkan etc. mittels Smart Objects zum Leben erweckt werden. Dadurch werden klassische Bücher, Lerngegenstände oder Spielzeuge multimedial erweitert, so dass interaktive Lernformen möglich werden. Im Kontext der vorliegenden Forschungsarbeit werden diese Smart Objects also Bestandteil der Lernräume, indem ausgewählte Dinge im Raum und insbesondere die Lerngegenstände digitalisiert und mit IT angereichert werden. Auf diese Weise werden normale Gegenstände eines Lernraumes in intelligente Dinge transformiert, die wiederum mit anderen Dingen vernetzt sind und untereinander kommunizieren können (vgl. Abschnitt 2.3.3).

\subsubsection{Internet der Dinge und Embedded Systems}

Die Idee, dass eingebettete „Minicomputer“ die Menschen unauffällig in ihrem Alltag unterstützen hat viele visionäre Vorläufer, welche nicht nur aus dem Science-Fiction Genre bekannt sind. Mark Weiser beispielsweise war ein USamerikanischer Wissenschaftler (1952-1999) aus den Bereichen Informatik und Kommunikationswissenschaften, der o. a. Visionen sehr detailliert in seinem bekannten Aufsatz „The Computer for the 21st Century“ aus dem Jahr 1991 formulierte. In diesem Zusammenhang werden oft auch Begriffe wie Pervasive

\footnotetext{
${ }^{3}$ https://www.tiptoi.com/
} 
Computing und Ubiquitous Computing genannt, welche sich auf eine allesdurchdringende Vernetzung des Alltags durch den Einsatz intelligenter Gegenstände und auf eine allgegenwärtige Informationsverarbeitung beziehen. Der Autor Mark Weiser (1991, S. 94) beschreibt dies bereits vor knapp 30 Jahren folgendermaßen:

"Specialized elements of hardware and software, connected by wires, radio waves and infrared, will be so ubiquitous that no one will notice their presence."

In seiner Vision ist das Internet unsichtbar in die Umgebung integriert, so dass es von den Menschen nicht mehr wahrgenommen wird. Es unterstützt die Menschen kontinuierlich in ihren täglichen Routinen und das sogar ohne Verfahren der künstlichen Intelligenz nutzen zu müssen. Zwei wesentliche Merkmale seien dafür notwendig. Zum einen müsse der genaue Ort und zum anderen der Kontext erkannt werden. Über sogenannte „Badges“ könnten zum einen der Ort und auch die damit verbundene Person über konkrete IDs gekoppelt werden, sodass der Raum erkennt, wer sich in ihm befindet. Weiser formuliert insbesondere Anwendungsszenarien aus der Arbeitswelt, in denen Lernen und Arbeiten durch physische Räume wie folgt unterstützt werden könnten:

„Meetings, for example, consist of several people spending time in the same room, and the subject of a meeting is most probably the files called up on that room's display screen while the people are there. No revolution in artificial intelligence is needed, merely computers embedded in the everyday world."

(Weiser 1991, S. 99)

Darüber hinaus beschreibt er weitere Anwendungsszenarien insbesondere in Konferenzräumen für unterschiedliche Arten von Screens oder auch Tafeln, die eine neue Form der Zusammenarbeit ermöglichen sollen. Globale und virtuelle Zusammenarbeit und das gemeinsame Bearbeiten unterschiedlichster Dateien sind nach Weiser dabei die wesentlichen Aspekte eines ubiquitären Internets. Ziel sei es dabei, das Problem des ,information overload“ zu lösen, indem automatisierte und bedarfsorientierte Suchen die Menschen in ihrer täglichen Arbeit unterstützen. Nützliche Mehrwerte generieren sich hierbei nicht nur durch die Nutzung digitaler Displays oder weiterer Komponenten, sondern vielmehr durch deren Interaktion untereinander. Auch die Ausstattung der Räume mit entsprechenden Softwaretools bieten nach Weiser wertvolle Zusatzdienste innerhalb einer komplexer werdenden Arbeit (vgl. ebenda, S. 100).

Anhand eines Beispiels, bei welchem eine Person am Wochenende in der Zeitung einen interessanten Absatz liest und diesen mittels eines Stiftes digitalisiert 
und per E-Mail ins Büro schicken lässt, beschreibt Weiser eine weitere Vision ubiquitärer Technologie, die auf eine Verbindung von informellen und formalen Lernsettings ausgerichtet ist. Herausforderungen sieht Weiser insbesondere im Bereich der Vernetzung unterschiedlichster Devices mit proprietären Protokollen sowie im Datenschutz und der Datensicherheit. Der Autor weist zudem darauf hin, dass demgegenüber die technische Entwicklung der Hardware kein Problem darstellen würde.

Den ersten Visionen Weisers zufolge geht es beim Internet der Dinge darum, dass die Ausstattung mit technischer Intelligenz quasi unsichtbar erfolgt und die Menschen unauffällig umgibt. Beispielsweise durch eine Integration in unsere räumliche Umgebung durch SmartHome-Applikationen oder auch durch tragbare Wearables. Sie sind eine Konkretisierung des Ubiquitous Computing, der Allgegenwart der Datenverarbeitung, und ein Teil des Internets der Dinge. Sinn und Zweck ist meist die Unterstützung einer Tätigkeit in der realen Welt, etwa durch (Zusatz-)Informationen, Auswertungen und Anweisungen.

Unter Wearables versteht man Computertechnologien, die am Körper getragen werden können und i. d. R. in Kleidungsstücke wie Mützen ${ }^{4}$, Helme ${ }^{5}$, Bril$l^{6}{ }^{6}$, Uhren oder Schuhe integriert sind. Insbesondere im Fitnessbereich ${ }^{7}$ wird den Wearables aktuell ein großes Potenzial zugeschrieben. Gleichzeitig warnen Branchenverbände wie Bitkom vor einem Missbrauch persönlicher Daten, die für Fitness- und Gesundheitszwecke erhoben und verarbeitet werden.

Bei der Verarbeitung der besonders sensiblen Gesundheitsdaten müssen laut Bitkom die höchsten Standards für Datenschutz und technische Sicherheit der Geräte eingehalten werden. So sollte zum Beispiel die Speicherung und Auswertung der Daten für Nutzer*innen so transparent wie möglich erfolgen und Daten nur nach Einwilligung an Dritte weitergegeben werden. Bei der technischen Sicherheit sollte insbesondere die drahtlose Übertragung von Daten, zum Beispiel zwischen Fitness-Armband und einem Smartphone, verschlüsselt erfolgen (Maas \& Rohleder, 2016). Zudem sollten sich die Geräte untereinander eindeutig identifizieren und authentifizieren, um zu verhindern, dass Daten einfach abgegriffen werden können (vgl. Abschnitt 2.2.1.6).

Eine zweite Besonderheit des Internet der Dinge ist folglich eine unauffällige Integration von IoT-Komponenten in unseren Alltag. Aber was genau kann

\footnotetext{
${ }^{4}$ https://www.earebel.com/

${ }^{5}$ https://daqri.com/products/smart-helmet/

${ }^{6}$ https://www.computerbild.de/artikel/cb-News-PC-Hardware-Die-spannendsten-VirtualReality-Brillen-10945247.html

${ }^{7}$ https://www.sensoriafitness.com/
} 
man unter „IoT-Komponenten“ verstehen? Damit sind sogenannte eingebettete Systeme (Embedded Systems) gemeint. Im Kontext der vorliegenden Forschungsarbeit werden diese eingebetteten Systeme also Bestandteil der Lernräume, wodurch sich herkömmliche Räume in ,,intelligente“ Räume transformieren lassen (vgl. Abschnitt 2.3).

Nach Siemers und Rosenlechner (2011) werden unter eingebetteten Systemen (Embedded Systems) Computersysteme verstanden, die aus Hardware und Software bestehen und in komplexe technische Umgebungen eingebettet sind. Diese Umgebungen sind meist maschinelle Systeme (Informationssysteme), in denen das eingebettete System mit Interaktion durch einen Anwender arbeitet oder auch vollautomatisch (autonom) agiert. Die eingebetteten Systeme übernehmen komplexe Steuerungs-, Regelungs- und Datenverarbeitungsaufgaben für bzw. in diesen technischen Systemen (Siemers \& Rosenlechner, 2011). Die Autoren orientieren sich bei ihrer Definition an der Publikation von Scholz (2006).

Darüber hinaus formulieren Berns et al. (2010) differenziertere Merkmale eingebetteter Systeme, die im Folgenden aufgeführt werden.

\section{1. kennzeichnende Merkmale}

- fester Bestandteil eines technischen Systems

- Zweckbestimmtheit (im Gegensatz zum Universalrechner)

- Interaktion mit Umgebung durch Sensorik und Aktuatorik

- Reaktivität, meistens Realzeitabhängigkeit

2. sekundäre Merkmale

- oft für Regelungs- / Steuerungsaufgaben vorgesehen

- häufig Massenware, Konsumgut, billig (,Kommodität“)

- vielfach schlecht bzw. nicht wartbar und nicht erweiterbar

- für viele unverzichtbar, manchmal auch sicherheitskritisch

- zunehmend auch vernetzt (ubiquitär)

Eine Welt ohne Embedded Systems ist kaum noch vorstellbar, da sie insbesondere im Bereich der Mobilität zum Standard geworden sind. In Passagierflugzeugen, Fernzügen oder auch Autos sind Embedded Systems in Form von Bordcomputern, Kontrollsystemen oder anderen Standardbausteinen integriert, die eine sichere und zuverlässige Reise garantieren (Broy, 2010).

Embedded Systems sind sozusagen als intelligente Steuerzentrale die technische Grundlage für komplexere IoT-Netzwerke, die über den Mobilitätsbereich 
hinweg auch andere Wirtschaftsbereiche prägen, wie z. B. den Gesundheitsbereich. Ohne Embedded Systems könnten moderne medizinische Geräte - vom Herzschrittmacher über ein Endoskop bis zum Computer-Tomographen, keine diagnostischen Befunde erzeugen.

\title{
2.2.1.3 Internet der Dinge und Cyber-Physical Systems
}

Im Zusammenhang eingebetteter Systeme wird auch oftmals der Begriff CyberPhysischer-Systeme (CPS) verwendet. Auf diese geht Georg Schütte in einer Keynote-Rede anlässlich des acatech-Symposiums Cyber-Physical Systems am 02.02.2010 in München wie folgt ein:

\begin{abstract}
„Embedded Systems der Zukunft sind keine Einzelsysteme mehr, sondern grundsätzlich miteinander vernetzt. Dies ist auch ein ganz wesentlicher Grund für den neuen Begriff der „Cyber-Physical Systems“. Vernetzung erfordert Gemeinsamkeit: gemeinsame Standards und Kommunikationsprotokolle, und die dazu nötigen Architekturen.“
\end{abstract}

(Broy, 2010).

Entsprechend zielen CPS auf die Verbindung eingebetteter Systeme mit weltweiten Netzen. Damit entsteht zum einen eine direkte Verbindung (und Rückkopplung) zwischen der physischen und der digitalen Welt und zum anderen werden komplett neue Funktionszusammenhänge möglich. Mit der Vernetzung in einem globalen Netz, werden völlig neuartige Systemfunktionalitäten möglich, die erst noch erfunden werden müssen. Ein besonderes Potential basiert auf der Funktionsintegration. Diese ist auf die Multifunktionalität der Systeme fokussiert, wobei das Smartphone das herausragendste Beispiel darstellt. Diese Bündelung von Funktionalitäten wird erst durch die Anbindung an globale Netze wie dem Internet möglich. Darüber hinaus werden die mobilen Devices mit immer besseren Basistechnologien ausgestattet, die vor einigen Jahren aufgrund der hohen Kosten noch undenkbar gewesen wären (vgl. Moorsches Gesetz Kapitel 1). Eine immer ausgefeiltere Sensorik und Aktuatorik dieser Geräte bietet eine optimale Grundlage, um diese als IoT-Schnittstelle auch für Lehr- und Lernprozesse zu nutzen und in Lernräumen strategisch zu integrieren.

Um ein ganzheitliches Bild über den Begriff Internet der Dinge und die Zusammenhänge zwischen Embedded- und Cyber-Physical Systems zu zeichnen, werden abschließend auch für CPS die wesentlichen Merkmale aufgeführt (vgl. ebenda).

- direkte Verbindung zwischen physischer und digitaler Welt 
- neuartige Systemfunktionen durch Informations-, Daten- und Funktionsintegration

- Funktionsintegration ermöglicht Multifunktionalität

- Zugriff über Netze überregional, ortsungebunden

- weiche bis harte Zeitanforderungen

- umfangreiche Interaktion

- Netze von Sensoren und Aktuatoren

- Vernetzung innerhalb der Systeme und nach außen

- dedizierte Nutzerschnittstellen: Starke Integration in Handlungsabläufe

- Einsatz unter oft schwierigen Randbedingungen

- Langzeitbetrieb

- Automatisierung, Adaptivität, Autonomie

Darüber hinaus formuliert Broy (2010) hohe Anforderungen an folgende Aspekte:

- funktionale Sicherheit

- Zugriffssicherheit und Datenschutz

- Zuverlässigkeit

- hoher Kostenduck

Als eine dritte Besonderheit des Internet der Dinge kann daraus resultierend die Vernetzung von unterschiedlichen Embedded Systems zu einem Cyber-Physical System abgeleitet werden. Im Kontext der vorliegenden Forschungsarbeit bedeutet dies, dass die eingebetteten Systeme als Bestandteil der Lernräume, mit anderen Informationssystemen gekoppelt werden. Dies ermöglicht die Entwicklung neuartiger Funktionen und Services im Lernraum im Sinne einer Multifunktionalität (vgl. Abschnitt 2.3).

\subsubsection{Internet der Dinge und die technologische Konvergenz}

Allen Definitionen und Beschreibungen zum Begriff Internet der Dinge liegt darüber hinaus zugrunde, dass sich die Funktionalität des Internet der Dinge aus einer Kombination unterschiedlicher Technologien ergibt, welche in ihrer Gesamtheit eine neue Qualität der Informationsverarbeitung entstehen lässt (u. a. Abicht et al., 2010; Fleisch \& Thiesse, 2014).

Technologische Konvergenz bedeutet, dass das Internet der Dinge keine geschlossene oder eigenständige Technologie bezeichnet, sondern vielmehr die Zusammenführung zahlreicher Technologien aus unterschiedlichen Feldern wie 
z. B. Informationstechnik, Elektronik, Sensorik, Werkstoffforschung, Kognitionswissenschaften etc. zu einem an der Anwendung orientierten Gesamtsystem darstellt. Die informationstechnische Vernetzung versetzt selbst bis dato proprietäre Systeme in die Lage, Informationen systemübergreifend zwischen Personen und Gegenständen bzw. zwischen Gegenständen untereinander auszutauschen. Gemäß einer während der deutschen EU-Ratspräsidentschaft im Jahr 2007 entwickelten Definition

\begin{abstract}
„,ist das Internet der Dinge die technische Vision, Objekte jeder Art in ein universales digitales Netz zu integrieren. Ausgestattet mit einer eindeutigen Identität, befinden bzw. bewegen sie sich in einem ,intelligenten“ Umfeld, wodurch eine Verbindung zwischen der physischen Welt der Dinge und der virtuellen Welt der Daten geschaffen wird. Während bislang noch überwiegend Computer und andere Netzwerkgeräte über eine Identität im weltweiten Internet verfügen, werden zukünftig auch zahlreiche Alltagsgegenstände wie z. B. Autos, Konsumgüter, Stromzähler, Objekte im Gesundheitswesen oder sogar Kleidungsstücke über das Netz angesteuert werden und selbständig miteinander kommunizieren können."
\end{abstract}

(Horvath 2012).

Eben aufgeführte Formulierung spiegelt sich in vielen Begriffsbestimmungen wider, wobei die Substantive „,von technischer Vision“ über ,technische Konzepte“ bis zur „globalen Infrastruktur" reichen. An dieser Stelle wird deutlich, dass wir uns derzeit an einem Wendepunkt befinden, an welchem die Entwicklung derartiger Systeme noch am Anfang steht und vollumfassende, autonom und intelligent agierende IoT-Infrastrukturen noch eher Prototypen-Status besitzen.

Denn IoT-Systeme sind nicht per se ,intelligent“. Die Intelligenz entsteht erst in einem hoch komplexen Prozess der technologischen Konvergenz, nämlich in der Vernetzung von Smart Objects zu Embedded Systems sowie mit anderen Netzwerken zu informationsverarbeitenden Gesamtsystemen, den Cyber-Physical Systems. Erst dadurch können die „vernetzten Dinge“ selbständig Informationen aus den entsprechenden Netzwerken generieren, austauschen, auswerten und neue Daten erzeugen, was bisher ein Privileg der Menschen war.

"Each definition shares the idea that the first version of the Internet was about data created by people, while the next version is about data created by things."

(Technopedia 2015)

Eines der größten Probleme, die mit der zunehmenden Gerätevernetzung auf Basis unterschiedlicher Technologien einhergehen, ist die mangelnde Kompatibilität. Dies betrifft die einzelnen Branchen wie z. B. Haustechnik, Gesundheit, Logistik, 
Produktion und insbesondere natürlich eine branchenübergreifende Vernetzung. Derzeit haben Hersteller und oft auch einzelne Geräteserien bzw. Technologietypen ihre individuellen und proprietären Schnittstellen. Um dieser Entwicklung entgegenzuwirken haben sich verschiedene Wirtschaftsbereiche und Branchenvertreter in Arbeitskreisen und Netzwerken zusammengeschlossen. Ziel ist die Schaffung breiter offener Standards, die es ermöglichen, Geräte verschiedener Hersteller und Technologien miteinander bedarfsgerecht zu kombinieren.

Ein flüssiger Informationsaustausch erfolgt entweder lokal in geschlossen ITSystemen (z. B. Feldbus, Intranet) oder auch global über das Internet. In diesem Zusammenhang ist die Entwicklung des Internet Protocol Version $6\left(\right.$ IPv6 $\left.^{8}\right)$ von besonderer Relevanz, da faktisch jedem ,Objekt“ im Internet der Dinge eine eindeutige IP-Adresse zugewiesen werden muss. Aufgrund der enorm hohen Anzahl an benötigten Einzeladressen ist IPv6 zumindest technologisch betrachtet in der Lage, das Internet der Dinge allgegenwärtig werden zu lassen. Heise-Online verweist diesbezüglich auf einen gigantischen IPv6-Adressraum, nämlich $2^{128}$ bzw. rund 340,28 Sextillionen. Das genügt, um jeden Quadratmillimeter der Erdoberfläche inklusive Ozeanen mit rund 600 Billiarden Adressen zu bepflastern (Zivadinovic, 2007).

Autonome, intelligent gesteuerte Abläufe werden erst dadurch erreicht, dass nicht nur Dinge und Maschinen in einem Netzwerk untereinander (global) kommunizieren, sondern noch komplexere, automatisierte Vorgänge durch die Auswertung von Daten in Echtzeit (Big Data) sowie durch die Berücksichtigung und Integration in Geschäftsprozesse durchgeführt werden können. Die Autoren Galis \& Gavras (2013) unterscheiden in diesem Zusammenhang zwischen M2M(Machine-to-Machine) und M2B- (Machine-to-Business) Kommunikation:

„More sophisticated approaches go beyond simple communication integration and target more complex interactions where collaboration of devices and systems is taking place. The cross-layer interaction and cooperation is pursued at

- machine-to-machine (M2M) level where the machines cooperate with each oth-er (machine focused interactions)

- machine-to-business (M2B) level where machines cooperate also with network-based services and business systems (business service focus).“

\footnotetext{
${ }^{8}$ https://www.teltarif.de/internet/ipv6/datenschutz.html
} 
Eine besonders umfassende Definition, die alle oben beschriebenen ,intelligenten Teilaspekte“ des Internet der Dinge berücksichtigt ist die folgende:

"IoT is a dynamic global network infrastructure with self configurating capabilities based on standard and interoperable communication protocols where physical and virtual ,things“ "have identities, physical attributes and virtual personalities, use intelligent interfaces and are seamlessly integrated into the information network."

(Vermesan \& Friess 2015).

Erst durch das intelligente Zusammenwirken ${ }^{9}$ vieler einzelner Objekte werden sinnvolle und effizientere Lösungen für den Menschen realisierbar:

"When many objects act in unison, they are known as having ambient intelligence."

(Technopedia 2015)

In eben diesem intelligenten, aufeinander abgestimmten Zusammenwirken zwischen Dingen, Technik und Prozessen liegt die besondere Herausforderung des Internet der Dinge, um aus ,nice to have“- Anwendungen sinnvolle und nachhaltige Lösungen zu entwickeln und um bestehende (globale) Strukturen und Prozesse zu optimieren.

Als eine vierte Besonderheit des Internet der Dinge lässt sich resultierend eine branchen-, system- und geräteübergreifende Vernetzung durch technologische Konvergenz ableiten. Im Kontext der vorliegenden Forschungsarbeit bedeutet dies, dass intelligent gesteuerte Abläufe in Lernräumen erst stattfinden können, wenn a) eine technologische Konvergenz vorhanden ist und b) eine für den Lehrenden bzw. Lernenden sinnvolle Verknüpfung mit digitalen Lern- und Geschäftsprozessen stattgefunden hat. (vgl. Abschnitt 2.3).

\subsubsection{Internet der Dinge und das Future Internet}

Weiterführende Konzepte des Internet der Dinge werden mit dem Begriff des „Future Internet“ umschrieben. Charakteristisch für das sogenannte Future Internet ist, dass im Sinne einer technologischen Konvergenz verschiedene IoT-Ebenen kombiniert und vernetzt werden (vgl. Abbildung 2.4):

1. Internet der Nutzer (Internet of People)

2. Internet der Inhalte (Internet of Content)

3. Internet der Dinge (Internet of Things)

${ }^{9}$ Vgl. Video von IBM auf YouTube: https://www.youtube.com/watch?v=sfEbMV295Kk 
4. Internet der Dienste (Internet of Services)

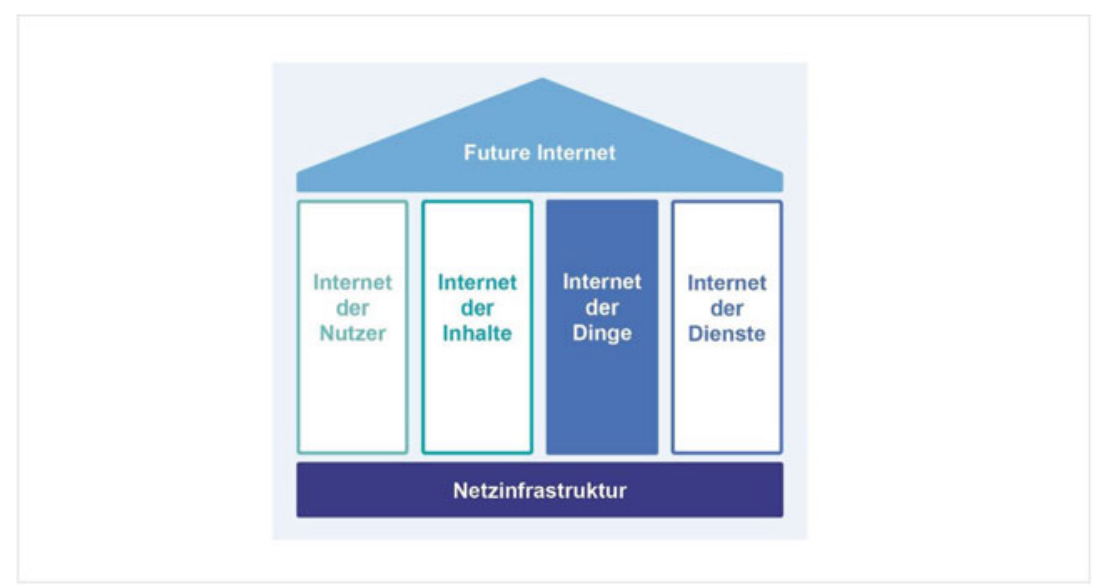

Abbildung 2.4 Das Internet der Dinge als Teil des Future Internet (Quelle: Eigene Darstellung in Anlehnung an die Europäische Kommission 2008)

Dabei ist das Internet der Dinge in der Lage, Personen, Dinge und Geräte miteinander zu verbinden und bettet sich in das derzeit ebenfalls entstehende Szenario für ein zukünftiges Internet, das „,Future Internet“ ein, welches auf vier thematischen Säulen basiert, denen eine gemeinsame Netzwerkinfrastruktur zu Grunde liegt (Gabriel, Gaßner \& Lange, 2010).

Die zentrale Rolle des Internet der Dinge wird in den Diskussionen um das Future Internet betont, wobei eine klare Abgrenzung zwischen ,Internet der Dinge“ und „Future Internet“" schwierig ist, da die Begriffe in ähnlichen Kontexten genutzt werden und auch ähnliche Ziele verfolgen (globale Vernetzung, neue Geschäftsmodelle, Effizienz etc.).

Nach Galis \& Gavras (2013) ist das Internet der Dinge die technologische Basis, auf Grundlage derer Funktionen eines Future Internet ${ }^{10}$ erst möglich werden. Ihrer Meinung nach bettet sich das Internet of Things wie folgt in den Kontext des Future Internet ein:

\footnotetext{
${ }^{10}$ Nähere Erläuterungen zum „Future Internet“ sind dem Video des Semantic Technology Institute International - STI2 auf YouTube zu entnehmen: https:/www.youtube.com/watch? $\mathrm{v}=$ off08As3siM
} 
"Internet of Things is seen as a key part of the Future Internet vision which will enable real-time interaction with the physical environment.

Billions of connected heterogeneous devices, sensing and actuating the physical environment in which they are embedded, and interacting among them or with remote users comprise the foundation of IoT."

Das Future Internet wird hierbei beschrieben als ein hoch komplexes System von heterogenen Einzelsystemen, welches durch folgende Eigenschaften gekennzeichnet ist:

- präzises Echtzeit-Monitoring

- Entscheidungsfindung aufgrund komplexer Data-Analytics

- zeitnahes Management

Insbesondere im Logistik- und Produktionsbereich (Industrie 4.0) lassen sich durch o.a. technologischen Funktionalitäten enorme Vorteile erkennen, welche in zahlreichen Studien hinsichtlich ihres wirtschaftlichen Potentials näher untersucht wurden (Kelkar et al., 2014). Die wirtschaftliche Leistungsfähigkeit des Internet der Dinge wird im Anwendungsbereich Industrie 4.0 als extrem hoch prognostiziert. Dadurch fungiert das Technologiekonstrukt Internet der Dinge als einer der wichtigsten Treiber des deutschen Wirtschaftsstandortes:

"Rob Lloyd von Cisco beziffert die Geschäftsmöglichkeiten im Internet der Dinge bis zum Jahr 2020 auf 14 Billionen \$.”

(Andelfinger \& Hänisch 2015).

Der Grund für diese milliardenschwere IoT-Markteinschätzung liegt wohl einerseits daran, dass bereits heute eine Vielzahl von Menschen mittels mobiler Devices kontinuierlich mit dem Internet verbunden sind und diese Anzahl drastisch zunimmt. Demnach ist es annähernd egal, ob es sich um 28, 32 oder 33 Milliarden vernetzter Geräte handelt.

Mit zunehmender Zahl werden Geräte und Sensoren einen beständig anschwellenden, nie gekannten Datenstrom erzeugen. Extrem große Informationsmengen müssen gesammelt, analysiert und gespeichert werden (Siemens, 2014a).

Andererseits zeichnet sich das Internet der Dinge durch interdisziplinäre Anwendungsbereiche aus, d. h., dass die beschriebenen Technologien in unterschiedlichsten Wirtschaftsbereichen angewendet werden können und dadurch enorme Marktpotentiale ermöglicht. Laut Cisco soll das Internet der Dinge bis 2020 auf ca. 50 Milliarden Dinge anwachsen (Cisco, 2015). 
Als eine fünfte Besonderheit des Internet der Dinge lässt sich festhalten, dass den IoT-Systemen eine zentrale Bedeutung im Rahmen zukünftiger Szenarien wie dem Future Internet sowie innerhalb wirtschaftlicher Gesamtentwicklungen in Deutschland allgemein beigemessen wird. Im Kontext der vorliegenden Forschungsarbeit bedeutet dies, dass IoT auch vielfältige Chancen und Potentiale für den Bildungssektor vermuten lässt, auch wenn diese bisher kaum beleuchtet bzw. empirisch erforscht wurden. Visionäre Anwendungen im Bereich des Wissensmanagements müssen neu gedacht und innoviert werden. In Frage kämen hier adaptive und ubiquitäre Lernanwendungen wie z. B. durch präzises Echtzeit-Monitoring, On Demand Empfehlungen zu Lerninhalten aufgrund komplexer Learning-Analytics sowie ein zeitnahes, ortsunabhängiges, adaptives Lern-Management, das informelle und formale Lernprozesse gleichermaßen organisiert (vgl. Abschnitt 2.3).

\subsubsection{Internet der Dinge und der Datenschutz}

Die im vorhergehenden Abschnitt prognostizierten Potentiale bergen jedoch nicht nur Chancen, sondern auch ein beachtliches Risiko. Fragen zum Datenschutz und zur Datensicherheit werden in nahezu allen Artikeln zum Internet der Dinge erwähnt (vgl. Vermesan \& Friess 2015; Andelfinger \& Hänisch 2015; Elektronik-Journal 2015; Cisco 2015).

Durch das Internet der Dinge steigt die Anzahl vernetzter Geräte in der Cloud, dadurch haben potentielle Angreifer neue Möglichkeiten die Systeme z. B. durch Malware $^{11} \mathrm{zu}$ manipulieren oder sogar gänzlich $\mathrm{zu}$ zerstören. Um das zu verhindern, sind starke Kryptografie und sichere IT-Konzepte gefragt. Spezielle Hardware-Lösungen helfen, Schlüssel sicher zu speichern und Änderungen am Programm-code zu verhindern.

Das Internet der Dinge bietet jedoch nicht nur für ,externe Angreifer“ neue Möglichkeiten der Manipulation. Auch die Unternehmen selbst können diese Technologien nutzen, um eigene Produkte oder Services ,,besser erscheinen zu lassen" als sie tatsächlich sind.

Laut einer repräsentativen Umfrage des Instituts für Demoskopie Allensbach aus 2015 haben sich jedoch trotz der vielen Meldungen zu Hackerangriffen und Cyberkriminalität die in der Befragung geäußerten Sorgen der Bevölkerung hinsichtlich einer persönlichen Bedrohung nicht nennenswert erhöht, sondern im Vergleich zum Vorjahr sogar eher verringert.

\footnotetext{
${ }^{11}$ Als Schadprogramm, Schadsoftware oder Malware bezeichnet man Computerprogramme, die entwickelt wurden, um unerwünschte und gegebenenfalls schädliche Funktionen auszuführen. Malware ist damit ein Oberbegriff, der u. a. den Computervirus umfasst.
} 
Parallel zu offenen Fragen hinsichtlich Datenschutz und Datensicherheit ergeben sich zudem Lücken hinsichtlich einer gesetzlichen Grundlage. Weber (2013) konstatiert den aktuellen Handlungsbedarf wie folgt:

„The Internet of Things (IoT) as an emerging global Internet-based information architecture facilitating the exchange of goods and services is gradually developing.

While the technical aspects are being discussed in detail a legal framework does not exist so far."

Die EU-Kommission arbeitet mit Expertenteams an einer Ausgestaltung gesetzlicher Rahmenbedingungen (vgl. Abschnitt 2.2.3), welche einerseits neue Geschäftsmodelle und Funktionen legitimieren (z. B. vollautonomes, assistiertes Autofahren) und andererseits den Aspekten Datenschutz und Datensicherheit den entsprechenden Rechtsschutz bieten sollen. Folgende Teilbereiche werden diesbezüglich näher betrachtet und rechtliche Paragraphen derzeit ausgearbeitet (vgl. ebenda):

- Privacy and Data Protection

- Security and Safety

- Ethics

- Object Identifiers and Interoperability

- IoT Governance

- Standards for meeting policy objectives

\subsubsection{Herausforderungen und Kritik}

Zusammenfassend kann festgestellt werden, dass bereits heutzutage einige Produkte und Services aus dem Bereich Internet der Dinge Realität geworden sind und sich zunehmend auch komplexere Anwendungen herausbilden, die sich kontinuierlich weiterentwickeln.

Besonders auffallend ist, dass sich die Herausforderungen nicht ausschließlich auf technische Bereiche wie bspw. der Interoperabilität, Skalierbarkeit, Softwarekomplexität, Data Analytics, Energieversorgung, funkbasierter Kommunikationsstandards etc. beziehen, sondern sich vermehrt auch in sozialen Zusammenhängen manifestieren. Diese sind gekennzeichnet durch neue Anforderungen, mit denen Organisationen und deren Mitarbeiter*innen, Politik und Gesellschaft gleichermaßen konfrontiert werden.

Die Autoren Mattern und Flörkemeier (2010) verweisen diesbezüglich auf die Notwendigkeit eines wirklich Nutzen stiftenden Internet der Dinge, wozu es mehr als „einer nur mikroelektronischen Aufrüstung bedarf“ (vgl. ebenda, S. 120). 
So ist den Autoren zufolge auch die Auswertung von Daten keineswegs trivial, um auf Basis einer solchen Datenanalyse Nutzen, Sinn und Wert zu generieren. Kritisch gesehen werden neben dem Datenschutz und der Datensicherheit (vgl. 2.2.1.6) insbesondere auch Aspekte der Zentralisierung. Spezifische Autoritäten, die als regulatorische Macht das Internet der Dinge beherrschen würden, müssten demnach dringend unterbunden werden. Dezentrale Netze könnten derartige Formationen unterbinden. Die zunehmende Technologiedependenz wird als weitere Herausforderung aufgeführt. So argumentieren die Autoren Mattern und Flörkemeier (2010) weiter, dass durch eine zunehmende Vernetzung und Steuerung von alltäglichen Dingen über das Internet auch die Gefahr wachsen würde, bei Stromausfall oder gar „Cyberangriffen“ in eine unvorhergesehene Hilflosigkeit zu verfallen, da ohne Strom oder gar Internetanbindung die Systeme nicht mehr gesteuert werden könnten (vgl. ebenda, S. 119). Als Technikpaternalismus wird von den Autoren ein weiterer, bisher eher selten aufgeführter Kritikpunkt formuliert, der sich auf die kontinuierliche „Einmischung“ von technischen Geräten auf unser Leben bezieht, der in subtiler Weise das Leben komfortabler gestalten soll, jedoch nicht immer ,zum Guten“, sondern auch zu beispielsweise unnützen Impulseinkäufen führen könne (vgl. ebenda, S. 119).

Parallel zu eben skizzierten sozialen und gesellschaftspolitischen Herausforderungen kristallisieren sich auch neue Anforderungen durch das Internet der Dinge im organisationalen Kontext. Diese manifestieren sich in einer nicht ausreichenden Innovationsfähigkeit, die zur Entwicklung neuartiger IoT-Produkte und IoT-Services benötigt wird. Eine kontinuierliche Weiterbildung der Mitarbeiter*innen ist Grundstein jeder zukunftsorientierten IoT-Gestaltungsarbeit. Diese Weiterbildung muss dringend ganzheitlich betrachtet werden, da sich nicht nur technische, sondern auch soziale Bezüge und gesellschaftliche Rahmenbedingungen verändern.

Derzeit fehlt es an sinnvollen IoT-Use-Cases, die aus einer ,nice-to-have“ Funktionalität einen echten Mehrwert für die Nutzer*innen generiert. Aus diesem Grund erhalten designorientierte Innovations- bzw. Lernmethoden einen hohen Stellenwert innerhalb der digitalen Transformation (vgl. Abschnitt 3.3). Aufgrund der technischen wie sozialen Komplexität des Internet der Dinge müssen Menschen zunehmend in interdisziplinären Teams zusammenarbeiten, darüber hinaus müssen komplexe Sachverhalte und Zusammenhänge erkannt und verstanden werden.

Ein verantwortungsvolles und menschenzentriertes Internet of Things benötigt viele Kompetenzen in der Umsetzung. Darüber hinaus fehlt es an guten Beispielen und an den entsprechenden gesetzlichen Rahmenbedingungen. Die Gesellschaft 
steht diesbezüglich an einem Wendepunkt, da IoT-Systeme erst im Aufbau sind und Anwendungsfelder noch erforscht bzw. innoviert werden müssen.

Das Internet of Things realisiert sich wie in den vorhergehenden Abschnitten dargestellt in einzelnen Schritten, die in einem langsamen, iterativen Prozess beschritten werden, wobei technische wie soziale Faktoren in einem Gesamtsystem berücksichtigt und aktiv gestaltet werden müssen (vgl. Abbildung 2.5).

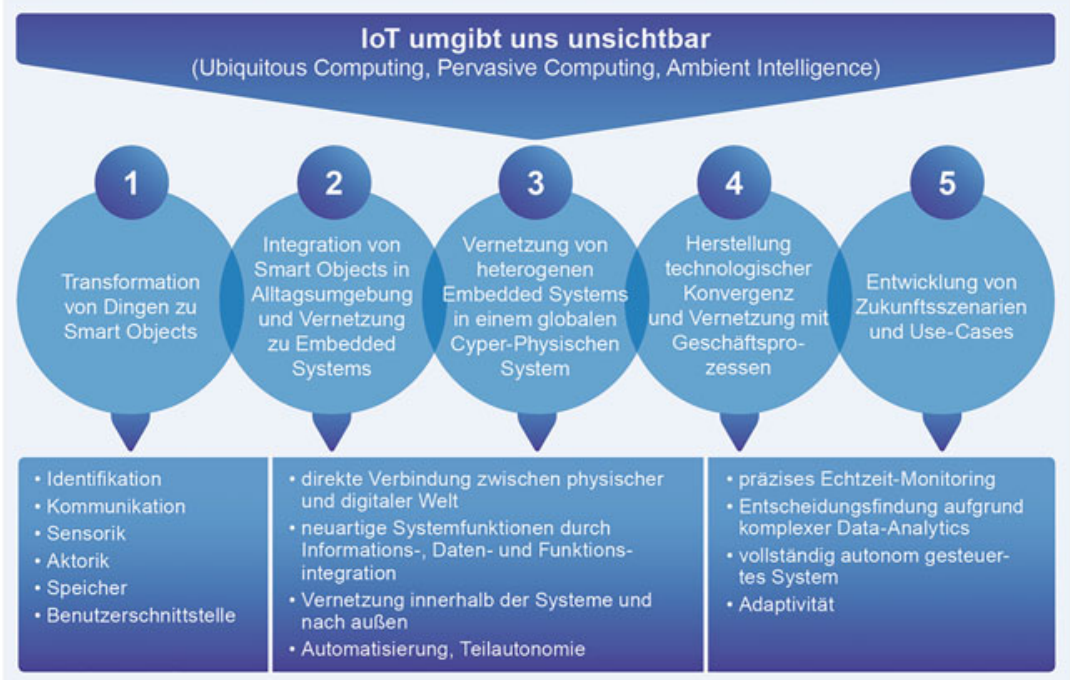

Abbildung 2.5 Reifegrade des Internet der Dinge (Eigene Darstellung)

Das Internet der Dinge ist nicht einfach da, es muss aktiv gestaltet werden. Die Funktionen und Use-Cases müssen strategisch geplant werden. Die Gestaltungsarbeit verläuft in einer wechselseitigen Abfolge zwischen Dingen, Technik und Prozessen. Bei der Entwicklungsarbeit bauen die Stufen zwar logisch aufeinander auf (Smart Objects, Embedded Systems, Cyber-Physische Systeme, technologische Konvergenz, Zukunftsszenarien), dennoch sind kontinuierliche Rückbezüge zu anderen Entwicklungsstufen notwendig, um das Gesamtsystem kontinuierlich $\mathrm{zu}$ verändern und an menschlichen Bedürfnissen anzupassen.

Ein IoT-System ist aus dieser Perspektive betrachtet nie abgeschlossen bzw. fertig, sondern es entwickelt sich stetig weiter. Da die Wirtschaft, Gesellschaft 
und Politik diesbezüglich noch am Anfang stehen, werden intelligent und autonom gesteuerte Abläufe auf Grundlage komplexer Cyber-Physischer Systeme erst in vielen Jahren Realität werden, die in o.a. Abschnitten im Rahmen des Future Internet beschrieben wurden. Die Vision des Future Internet gründet sich derzeit noch auf theoretischen Vorüberlegungen. Nichtsdestotrotz sind auch hier konkretere Entwicklungen und Umsetzungsprojekte in den nächsten 10 Jahren abzusehen.

Wichtig an dieser Stelle der IoT-Entwicklung ist es, die aktive Gestaltung dieses Prozesses auf allen Ebenen nachhaltig zu betreiben. Das bedeutet, dass soziale wie technische Aspekte gleichrangig berücksichtigt und ausgestaltet werden müssen. Dies impliziert die intensive Auseinandersetzung mit den Themenfeldern Datenschutz, Sicherheit, Interoperabilität, Gesellschaft und Ethik sowie Wirtschaft. Da das Thema Internet der Dinge auf der einen Seite durch hohe Komplexität gekennzeichnet ist und auf der anderen Seite in unterschiedlichste Anwendungs- wie Tätigkeitsbereiche eindringt, muss die Gestaltungsarbeit entsprechend transdisziplinär erfolgen. Zudem müssen die notwendigen gesetzlichen Rahmenbedingungen zeitnah diskutiert und zum Wohle der Gesellschaft ausgearbeitet werden. Darüber hinaus sollten IoT-Konzepte grundsätzlich an den Bedürfnissen der Menschen ausgerichtet werden, welches die Entwicklung sinnvoller und Nutzen stiftender Use-Cases ${ }^{12}$ beinhaltet.

Trotz zahlreicher Publikationen und Untersuchungen zum Thema Internet der Dinge fällt auf, dass der Anwendungsbereich der „Bildung“ nahezu komplett unbeachtet bleibt, obwohl auch dort massive Veränderungen abzusehen sind. In einem Interview aus 2014 beschreibt Prof. Elgar Fleisch diese Entwicklungen folgendermaßen:

„Die einfache Wissensvermittlung wird zum Nutzen aller automatisiert: etwa über didaktisch gut aufbereitete Videovorträge. Für die vertiefende Diskussion danach braucht man dann wieder den persönlichen Kontakt zum Lehrer oder Professor.“

Derartige Auswirkungen werden derzeit insbesondere im Bereich des Wissensmanagements diskutiert - hierzu gibt es vielfältige (Forschungs-)Aktivitäten (MOOCs, BarCamps, Kongresse etc.). Diese beziehen sich jedoch in der Regel „nur“ auf Veränderungen innerhalb des digitalen Lernens bzw. auf IT-gestütztes (online-)Lernen.

\footnotetext{
${ }^{12}$ Eine Übersicht zu Anwendungen aus dem Bereich Internet der Dinge ist in einer Präsentation von WHY own it auf Slideshare zu finden: https://de.slideshare.net/WHYownit/internet-ofthings-die-20-spannendsten-produkte-45901691? related=1
} 
Das Internet der Dinge bietet jedoch wie im vorliegenden Kapitel hergeleitet auch im Bildungskontext weit mehr Möglichkeiten zum Lernen, indem die Grenzen zwischen physischen Dingen und der digitalen Welt aufgelöst werden. IoT-Konzepte ermöglichen hybride Lernformate, die digitale und analoge Lernarrangements verbinden. Auf diese Weise können bisher ungeahnte und hoch innovative Lernumgebungen gestaltet werden.

Diese fehlende Perspektive wird im Rahmen der vorliegenden Forschungsarbeit eingenommen, um wichtige Anregungen für eine gestaltungs- und zukunftsorientierte Bildungsarbeit zu liefern.

\subsubsection{Das Internet der Dinge im Bildungskontext}

Der folgende Abschnitt dient als Brücke zwischen dem Internet der Dinge (vgl. Abschnitt 2.2) und dem Untersuchungsgegenstand intelligenter und hybrider Lernräume. Dabei wird zunächst auf Grundlage einer Literaturrecherche nachgezeichnet, welche wissenschaftlichen Publikationen sich auf welche Art und Weise mit der Anwendung des Internet der Dinge im Bildungsbereich befassen.

Die systematische Literaturrecherche wurde Ende 2015 durchgeführt, wobei das Vorgehen folgende Arbeitsschritte umfasste:

- Festlegung der primären Suchbegriffe auf
o „Internet der Dinge“ UND Bildung
o „Internet der Dinge“ UND Bildung UND lernen
o „Internet of Things“ AND education
o „Internet of Things“ AND education AND learning

- Recherche über das Internet, Google Scholar sowie über den ZIH WebVPN Service der TU Dresden, um auf alle Publikationen der Sächsischen Landesbibliothek - Staats- und Universitätsbibliothek Dresden (SLUB) zugreifen zu können (inkl. Fachdatenbanken)

- Identifizierung von 1 relevanten Schlüsselwerk von insgesamt 28 relevanten Publikationen

- Speicherung und Verschlagwortung der Fundstelle in einem Literaturverwaltungsprogramm (Zotero)

- Sichtung und Strukturierung der Quellen mit Hilfe eines systematischen Textanalyseverfahrens. Dabei wurde die SQ3R-Methode nach Robinson (1978) angewendet (vgl. Abbildung 3.63). 
Als problematisch erwiesen sich für die Recherche insbesondere folgende Punkte (vgl. Abbildung 2.6):

1. Pädagogisch orientierte Fachdatenbanken haben im deutschsprachigen Raum zu knapp 30 Ergebnissen geführt, die im Prinzip alle der in Unterkapitel 1.2 genannten BMBF-Früherkennungsinitiative FREQUENZ zuzuordnen waren.

2. Der überwiegende Teil der identifizierten Quellen beschäftigt sich mit neuen Kompetenzanforderungen. Das bedeutet, dass in den wissenschaftlichen Publikationen erforscht sowie beschrieben wird, welche Kompetenzen im Zusammenhang des Internet der Dinge in Zukunft benötigt werden.

3. Ein weiterer Teil der Quellen beschäftigt sich mit modernen Lernmethoden zur Vermittlung von IoT-Fachkenntnissen.

4. Ein Großteil der Publikationen ist in einen internationalen Kontext eingebettet und entsprechend auf Englisch.

5. Eine systematische Recherche zu allen international erschienenen Publikationen ist in der Umsetzung kritisch zu betrachten, da die Meta-Suchmaschinen wie SLUB, BASE etc. auf Datenbestände zurückgreifen, die von Portal zu Portal unterschiedlich sind. Zudem gibt es tausende Fachdatenbanken, die wiederum nicht unbedingt in den Meta-Suchmaschinen gelistet sind und einzeln ,abgesucht" werden müssten.

6. Einige interessante Publikationen wie beispielsweise der Horizon Report (vgl. Abschnitt 1.2.1) bieten zwar interessante Anknüpfungspunkte, werden jedoch aufgrund eines fehlenden wissenschaftlichen Peer-Review-Verfahrens nicht in den Datenbanken aufgeführt.

7. Aus Perspektive der Fachdisziplin Informatik konnten insgesamt 1299 „IoTPublikationen“ identifiziert werden. Davon beschäftigten sich lediglich 28 mit dem „Internet der Dinge“ im Bildungsbereich.

8. Von diesen 28 Quellen konnte lediglich 1 Publikation identifiziert werden, die sich explizit mit Anwendungsmöglichkeiten des IoT innerhalb von Lehr- und Lernprozessen beschäftigt (Domingo \& Forner, 2010). Daraus kann geschlossen werden, dass eine direkte Übertragung von IoT auf Bildungsprozesse aus einer gestaltungsorientierten Perspektive noch annähernd unerforscht ist.

9. Entsprechend gibt es kaum empirische Befunde über IoT im Bildungsbereich.

10. Es kann davon ausgegangen werden, dass sich aufgrund des erhöhten Interesses am Thema Internet der Dinge weitere Forschungsprojekte in der Durchführung befinden, zu denen noch keine Publikationen vorliegen. Eine erneute Recherche würde wahrscheinlich neue und relevante Quellen hervorbringen. 


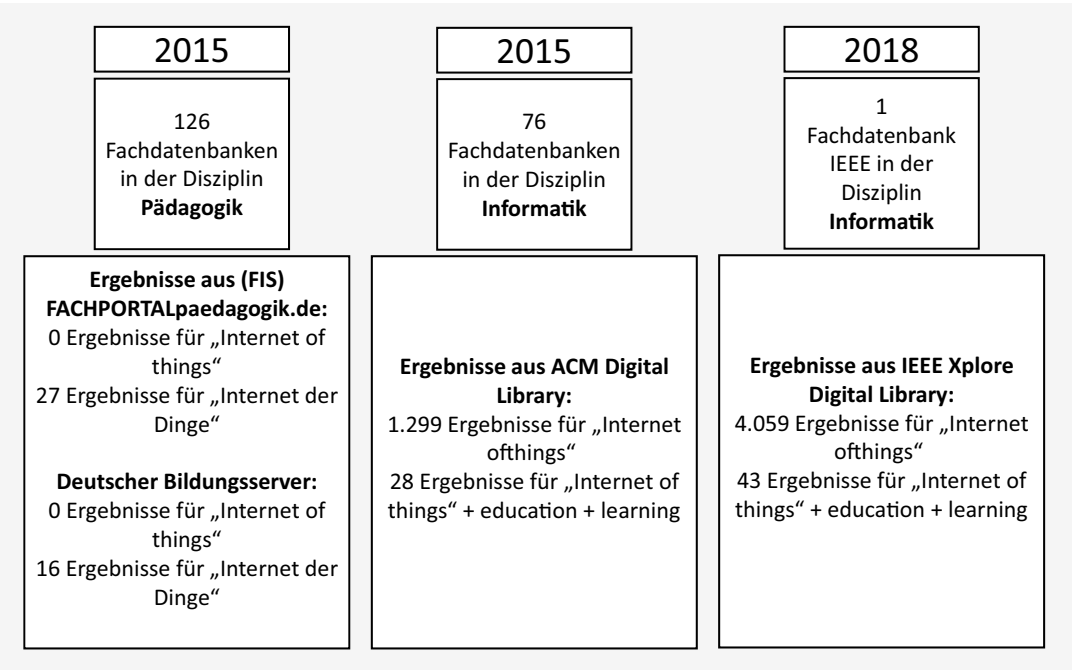

Abbildung 2.6 Anzahl der Rechercheergebnisse aus 2015 und 2018

Aufgrund der geringen Anzahl relevanter Fundstücke aus 2015 wurde im Frühjahr 2018 eine erneute Recherche zu oben aufgeführten englischen Suchbegriffen (,Internet of Things“ AND education AND learning) durchgeführt (vgl. Abbildung 2.6). Innerhalb der erweiterten Suche wurde der Begriff „Internet of Things“ als Titel im Dokument sowie die weiteren Suchbegriffe ,education“ und „learning“ als Metadaten in der Suchabfrage vorgegeben. Die Recherche wurde ausschließlich über die Online Datenbank IEEE Xplore durchgeführt.

Die Literaturanalyse umfasste eine Strukturierung der Fundstellen nach Veröffentlichungsjahr und eine Sichtung der jeweiligen Abstracts mit anschließender Kategorisierung in Publikationen, die sich

a) mit innovativen Bildungsformaten beschäftigten, indem das Internet der Dinge auf Lehr- und Lernprozesse übertragen wurde (und im Sinne der vorliegenden Arbeit als Schlüsselwerk zu bezeichnen ist)

b) mit der Vermittlung von IoT-Kompetenzen beschäftigten

c) in keine der beiden Kategorien zugeordnet werden konnten und sich mit ,sonstigen“ Schwerpunkten befassten (überwiegend IoT-Softwarearchitekturen) 


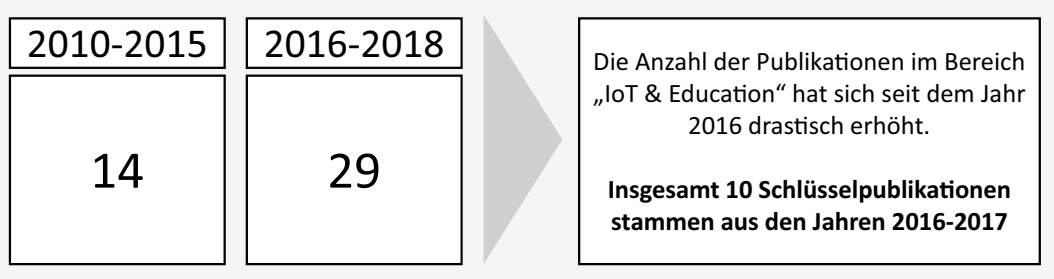

Abbildung 2.7 Anzahl der Rechercheergebnisse im Vergleich zwischen 2015 und 2018

Das Ergebnis der Literaturanalyse lässt sich in folgenden Punkten zusammenfassen:

1. Es konnten insgesamt 43 Publikationen identifiziert werden, die sich mit dem „Internet der Dinge“ im Bildungsbereich beschäftigen

2. Nahezu ein Drittel aller Fundstücke fokussiert auf die Vermittlung von IoT Kompetenzen (14 Publikationen)

3. Ein weiteres gutes Drittel beschäftigt sich mit sonstigen Themen des Internet der Dinge im Bildungsbereich, wobei überwiegend IoT Softwarearchitekturen adressiert werden (17 Publikationen)

4. Ein knappes Drittel widmet sich neuen Bildungsmethoden, indem das Internet der Dinge für innovative Lehr- und Lernprozesse genutzt wird (12 Publikationen). Insofern ist an dieser Stelle ein starker Anstieg der Publikationen im Vergleich zu 2015 zu verzeichnen.

5. Entsprechend konnten im Vergleich zu 2015 insgesamt 12 Schlüsselpublikationen im Frühjahr 2018 identifiziert werden

6. Auffällig ist, dass sich die Anzahl der Publikationen im Vergleich zu den Jahren zwischen 2010 bis 2015 im Kontext von IoT \& Education seit 2016 nahezu verdoppelt hat. (vgl. Abbildung 2.7).

7. Aus der wiederholten Literaturanalyse kann geschlussfolgert werden, dass das Thema „IoT in Education“ wie angenommen wichtiger und es zunehmend mehr Publikationen geben wird. Entsprechend kann auch mit weiteren empirischen Befunden über IoT im Bildungsbereich gerechnet werden. 


\subsubsection{Schlussfolgerungen}

Die im vorangegangenen Abschnitt aufgeführten Probleme hinsichtlich einer systematischen Annäherung an den Untersuchungsgegenstand hat dazu geführt, dass die Literaturreche aus 2015 um sekundäre Suchbegriffe aus dem IoT-Bereich (vgl. Abschnitt 2.2) erweitert wurde. Darüber hinaus hat sich gezeigt, dass ausschließlich englischsprachige Literatur relevante Quellen liefern.

Folglich wurde eine auf 2015 folgende Literaturrecherche im Frühjahr 2016 durchgeführt, wobei das Vorgehen folgende Arbeitsschritte umfasste:

- Festlegung der sekundären Suchbegriffe:

o Ubiquitous Learning

o Adaptive Learning

- Recherche über das Internet, Google Scholar sowie über den ZIH WebVPN Service der TU Dresden, um auf alle Publikationen der Sächsischen Landesbibliothek - Staats- und Universitätsbibliothek Dresden (SLUB) zugreifen zu können (inkl. Fachdatenbanken)

- Identifizierung von 49 relevanten Quellen

- Speicherung und Verschlagwortung der Fundstellen in einem Literaturverwaltungsprogramm (Zotero)

- Sichtung und Strukturierung der Quellen mit Hilfe eines systematischen Textanalyseverfahrens. Dabei wurde die SQ3R-Methode nach Robinson (1978) angewendet (vgl. Abbildung 3.63).

- Inhaltsanalytische Auswertung von 26 Schlüsselwerken (überwiegend aus dem englischsprachigen Raum)

- Die systematische Suche mit o. a Keywords wurde mit einem Schneeballverfahren kombiniert, d. h., dass die Suche über das Literaturverzeichnis einschlägiger Publikationen erweitert wurde. Besonders häufig zitierte Literatur wurde entsprechend den Schlüsselwerken zugeordnet.

Im Ergebnis dieser Literaturrecherche und -analyse zeigte sich, dass ein bis daher unbekanntes Lernformat auf Grundlage der wissenschaftlichen Publikationen identifiziert werden konnte, das Ubiquitous Learning und Adaptive Learning, in einem neuen Format zusammenführt. Dieses Lernformat wird als „Smart Learning Environment" bezeichnet. Smart Learning Environments (SLEs) sind physische Lernumgebungen, die mit adaptiven und kontextsensitiven digitalen 
Komponenten angereichert sind, um das Lernen effizienter zu machen (Koper, 2014).

Der Begriff Smart Learning Environments bündelt demzufolge die Funktionalitäten von intelligenten und hybriden Lernräumen (vgl. Abschnitt 2.3.3), die durch die Übertragung von IoT auf Lehr- und Lernprozesse möglich werden. Beim Begriff Smart Learning Environments handelt es sich um eine vergleichsweise neue Erscheinung, so dass es unterschiedliche Definitionen dazu gibt. Die vorliegende Forschungsarbeit bezieht sich auf die Definition nach Koper (2014):

„Smart learning environments (SLEs) are defined as physical environments that are enriched with digital, context-aware and adaptive devices, to promote better and faster learning.“

Auf dieser Grundlage aufbauend werden im Folgenden die Begriffe Smart Learning Environments und intelligente und hybride Lernräume synonym verwendet, wobei die bedeutungsgleiche Herleitung in Abschnitt 2.3.3 dezidiert erfolgt. In Abschnitt 2.3 wird gezeigt, dass es sich bei den Begriffen SLE und intelligente und hybride Lernräume um dieselben Konstrukte handelt. Insofern erfolgt im weiteren Verlauf der Forschungsarbeit die weitere Annäherung an den Untersuchungsgegenstand intelligenter und hybrider Lernräume über das Konstrukt der Smart Learning Environments, da für dieses Lernformat einschlägige, wissenschaftliche Publikationen vorliegen. Darüber hinaus kann auf diese Weise der Untersuchungsgegenstand geschärft und eingegrenzt werden.

Die detaillierte Literaturanalyse und die daraus resultierenden Ergebnisse werden in Abschnitt 2.4 im Rahmen des aktuellen Forschungsstandes zu Smart Learning Environments dargestellt und diskutiert.

\subsection{Lernräume}

Der Forschungsgegenstand der Lernräume wird im Folgenden zunächst aus der Perspektive einer bildungswissenschaftlichen Forschung beleuchtet, um theoretische Grundlagen zu bilden, die anschließend in den Kontext des arbeitsplatzbezogenen Lernens gestellt werden. Dadurch erweitert sich die Perspektive auf Lernräume im Arbeitskontext, wobei explizit auf Büroraumgestaltungsprinzipien eingegangen wird. Eine vollständige Darstellung des Forschungsstandes kann hier nicht erfolgen, da dies den Rahmen der Arbeit sprengen würde. Insofern werden unter dem Gesichtspunkt der „Gestaltungsprinzipen von Lernräumen“ allgemeine 
Empfehlungen zum Thema „Architektur für Erwachsenenbildung“ herausgearbeitet und aus unterschiedlichen Forschungshintergründen zusammengetragen. In einem finalen Schritt werden schließlich Merkmale intelligenter und hybrider Lernräume skizziert, um eine theoretisch begründete Brücke zum Begriff ,Smart Learning Environments" schlagen zu können.

\subsubsection{Raumtheoretische Grundlagen}

Für eine systematische Dekonstruktion des Begriffes „Lernraum“, wird zunächst das Konstrukt „Raum“ betrachtet. Sesink $(2007,2014)$ verweist im pädagogischen Kontext auf eine grundsätzlich metaphorische Verwendung des Raum-Begriffes, der von Spielraum, über Freiraum bis Weltraum und virtuellem Raum unterschiedliche Bedeutungen einnehmen kann. Im medienpädagogischen Bereich differenziert Kerres (2017) die Metaphern weiter aus und führt weitere Begriffe auf, die auf eine unscharfe Verwendung hindeuten. Diese reichen von Lernumgebung, Lernarrangement und Lernumwelt über mediale Bildungsräume oder Bildungsplattformen bis hin zu Ökosystemen.

Sesink (2007) weist zudem auf die Unterscheidung im Englischen hin, wobei zwischen „room“ als gestaltetem Raum und „space“ als ungestaltetem (leeren) Raum unterschieden werden muss. Weiterhin differenziert er die Potenzialität von Raum und bezieht sich dabei auf Winnicott (1974), die ,spielerischexperimentelle Begegnung von subjektiver Einbildungskraft" ermöglicht und damit Vorhandenes überschreitet. Die im weiteren Sinne architektonische Anlage von Räumen bezieht sich auf die Poietik von Räumen und impliziert damit den Aufforderungscharakter für bestimmte Handlungen. Schließlich kommuniziert der Raum auf symbolischer Ebene, z. B. Wertschätzung und Erwartungen an die Nutzenden. Die Raummetapher impliziert, dass Räume erfahrbar und gestaltbar sind. Sie sind von Menschen geschaffen unter Zuhilfenahme einer definierten Menge an Gestaltungsmöglichkeiten.

Mistele \& Trolle (2006) unterscheiden in ihrer Konstruktion von Lernräumen zunächst zwischen dem euklidisch-geometrischem Raumverständnis, das in den Dimensionen Breite, Höhe und Tiefe als objektiv „vermessbarer“ Raum definiert ist und einem wahrgenommenen Raum. Letzterer wird nach Bollnow (2010) als erlebter Raum bezeichnet. Dabei wird auf ein relativistisches Raumverständnis Bezug genommen, wonach der Raum sich permanent durch die ihn bildenden Objekte, Menschen und dessen Handlungen verändert. Bollnow stellt dabei den Menschen und dessen individuelle Wahrnehmung in den Mittelpunkt, in dem er die persönlichen, soziologischen und psychologischen Deutungen des Einzelnen 
mit einbezieht. Dies bedeutet, dass jeder Mensch Raum auf Grund seiner individuellen Wahrnehmung anders erlebt. Somit hat auch jeder Ort im erlebten Raum eine andere Bedeutung für den Menschen. Es gibt qualitativ unterschiedliche Orte im Raum, deren Beziehungen zueinander die Basis einer inhaltlichen Gliederung des erlebten Raums bilden (vgl. ebenda). Bollnow unterstellt damit eine konstruktivistische Perspektive, wonach die Wirklichkeit dem eigenen Erlebten, der Wahrnehmung und der Sinneserfahrung entspringt. Die Umwelten schaffen so über ihren Aufforderungscharakter Rahmungen, zu denen und in denen sich die Akteure verhalten.

Dementsprechend lassen sich zwei Handlungsfelder, Mensch und Raum, identifizieren, die sich in einer Dualität von Struktur und Handeln manifestieren. Der Raum ist sowohl Resultat als auch Bedingung sozialer Prozesse. Edinger (2015) spricht in diesem Zusammenhang auf Basis einer raumsoziologischen Begriffsklärung von einem materiell-sozial-virtuellem Konzept von Raum, wobei sich alle drei Komponenten eines Raumes (materiell, sozial, virtuell) bedingen und in Wechselwirkung miteinander stehen. Der materielle Raum (die erste Komponente) ist körperlich erfahrbar - also visuell, auditiv, haptisch und olfaktorisch. Die zweite Komponente ist der soziale Raum, er beschreibt in Anlehnung an Pierre Bourdieu (2007) das soziale Gefüge. Dieses Gefüge basiert u. a. auf Alter, Bildungsbiografie und Milieuzugehörigkeit der beteiligten Personen. Die Position im sozialen Raum wirkt sich auch auf die subjektive Wahrnehmung des Lernraumes und aller anwesenden Personen aus und darauf, ob man sich in diesem Lernraum wohl oder unwohl fühlt. Der virtuelle Raum ist ,eine metaphorische Bezeichnung“ für (Re-)Präsentationen des Internet im Raum. Dies können dreidimensionale virtuelle Welten, Augmented Reality, Videokonferenzsysteme, Touchscreens, Computer, Embedded Systems oder auch Kollaborationsplattformen wie Social Networks sein. Die virtuelle Komponente spielt im Hinblick auf intelligente und hybride Lernräume bzw. Smart Learning Environments für die vorliegende Forschungsarbeit eine bedeutende Rolle und wird in Abschnitt 2.3.3 näher erläutert.

Mistele \& Trolle (2006) erweitern die Lernraumperspektive, indem sie einen direkten Bezug zum selbstgesteuerten Lernen am Arbeitsplatz herstellen. Sie argumentieren, dass der Raum nicht mehr nur statischer Hintergrund, sondern selbst Teil von Lern- und Handlungsprozessen ist. In Verbindung mit dem arbeitsbezogenen Lernen stellt sich dann zwangsläufig die Frage, wie Räume des Lernens ausgestaltet sein müssen, um effektive Lernprozesse $\mathrm{zu}$ ermöglichen bzw. zu fördern.

Arbeitsbezogenes Lernen vollzieht sich unter anderem informell im Prozess der Arbeit (vgl. Abschnitt 2.1.2.3). Die Lernenden sind dabei aktive, gestaltende 
Menschen, die über unterschiedliche Lernpräferenzen verfügen und selbstgesteuert lernen. Dies schließt die konkrete, selbstbestimmte Wahl des Lernraums, der Lernmethoden, der Lernpartner und den Lernzeitpunkt mit ein (Mistele \& Trolle, 2006).

Soll der Raum vom Individuum als Lernraum erlebt und genutzt werden, muss er dessen Lernbedürfnissen und -präferenzen gerecht werden und frei wählbar sein. Die Menschen schaffen sich damit ihre individuellen Lernräume.

Edinger (2015) verweist auf dieselben Aspekte hinsichtlich Selbststeuerung und Nutzerzentrierung. Vor diesem Hintergrund plädiert sie dafür, auf Basis eines Human-Centered-Design-Ansatzes, die Bedürfnisse der Nutzer*innen gezielt zu erheben und diese Daten bzw. die Lernden selbst in den Prozess der Raumgestaltung aktiv einzubinden. Dabei erörtert sie die bedeutende Rolle von Learning/ User Journeys und Personas. Dies sind gängige Innovationsmethoden aus dem Design-Thinking, die sie wie folgt beschreibt (Edinger, 2015, S. 112-113):

„Es bietet sich an, Personas und User Journeys zu entwickeln. Eine Persona ist ein ausgestalteter Typus von Nutzern [...] im Sinne eines im untersuchten Feld anzutreffenden Menschen mit einem bestimmten soziodemografischen Profil, einem sozialen Kontext und biografischen Eigenschaften. Eine User Journey stellt den konkreten Ablauf im Umgang mit Produkten, Software, aber auch Architektur dar.“

So lassen sich beispielsweise formale oder informelle Lernformate und Lernprozesse in Lernräumen bzw. am Arbeitsplatz veranschaulichen. Dabei kommt es auf den konkreten Ablauf der einzelnen Tätigkeiten bzw. Lernaktivitäten an, die letztlich auch Auskunft über die Motivation des Lerners geben können. Ziel ist es, sich einen möglichst holistischen Überblick über die Zielgruppe(n) und deren Erfahrung im Umgang mit Lernarchitekturen zu verschaffen (vgl. ebenda).

Raum soll im Folgenden als materiell-sozial-virtuelles Konzept von Raum verstanden werden, der sich durch Kombination dreier miteinander verknüpfter Komponenten manifestiert. Dabei kann einer Dualität zwischen Subjektivität und Raumstruktur sowie dem erlebten Raum im Sinne sozialer Wirklichkeit am ehesten entsprochen werden. Darüber hinaus bietet die dritte Komponente konkrete Anknüpfungspunkte an den Forschungsgegenstand intelligenter und hybrider Lernräume.

Dieser theoretischen Grundlage folgend sind Lernräume in der vorliegenden Forschungsarbeit in einem Zusammenwirken folgender Ebenen zu betrachten:

1. Materielle Ebene

2. Soziale Ebene 


\section{Virtuelle Ebene}

Diese sind in ihrem kontinuierlichen Wirkungsgefüge untereinander verschränkt, können zu analytischen Zwecken jedoch auch einzeln betrachtet werden.

Essenziell für das Verständnis eines erlebten Raumes aus den genannten drei Komponenten ist, dass der Raum immer ein Zusammenspiel aus all diesen Komponenten ist. Sofern sich nur eine einzige Komponente verändert, wie etwa durch das Aussetzen des WLAN, so ändert sich der komplette Raum in der subjektiv wahrgenommenen Wirkung der Lernenden. Der Doppelcharakter von Struktur und Handeln bedingt explizit in Bezug auf Lernräume, dass die gleichen didaktischen Settings und Inhalte je nach Zusammensetzung der jeweils vorhandenen Raumkomponenten eine andere Wirkung entfalten können. Entsprechend argumentiert Erdinger (2015), nach Verhaltensmustern in ähnlichen Umgebungen zu suchen. Diese individuelle, sehr spezifische Recherche und Gestaltungsarbeit lässt sich dann systematisch mit Methoden aus dem Design Thinking wie z. B. Personas oder Learning Journeys begleiten.

Auf Basis dieser theoretischen Vorüberlegungen soll im nächsten Abschnitt die Perspektive der Lernraumforschung und Büroraumgestaltung eingenommen werden, um Gestaltungsempfehlungen identifizieren zu können.

\subsubsection{Lernraumforschung und Büroraumgestaltung}

Wie bereits in Abschnitt 1.2.2. dargelegt, konnte die Pädagogik noch keine fundierte Perspektive auf die Gestaltung von Lernräumen entwickeln. Sesink (2007, S. 2) formuliert das Desiderat wie folgt:

\footnotetext{
„Die architektonische Gestaltung von Lernräumen ist ein in seiner Bedeutung sträflich unterschätzter Teil pädagogischer Technik. Das Interesse für den Raum, das mit dem Vordringen der Neuen Technologien erwachte, ändert daran nichts. Eine der Diskussion um die notwendigen Qualitäten virtueller Lernräume auch nur annähernd vergleichbare Diskussion um die Qualitäten architektonischer Räume findet nicht statt.“
}

In Abgrenzung dazu kann festgestellt werden, dass sich insbesondere in den letzten Jahren ein großes Interesse zum Thema „Bildungsräume“ ausgebildet hat. Neuere Publikationen verweisen auf eine gestiegene Bedeutung des Themas (Arnold, Lermen \& Günther, 2015; Rummler, 2014), wobei sich eine deutliche Mehrheit mit virtuellen Lernräumen oder deren metaphorischen Bedeutungen auseinandersetzt. Die Gestaltung von physischen Lernräumen ist weiterhin 
als Spezifikum zu betrachten, auch wenn wissenschaftliche Untersuchungen diesbezüglich zugenommen haben. Von daher ist es ein Anliegen der vorliegenden Forschungsarbeit, auf die Gestaltung physischer Lernräume zu fokussieren, wobei die drei Raumkomponenten (materiell, sozial und virtuell) systematisch miteinander verwoben werden (vgl. Abschnitt 2.3.1).

Im Rahmen der Literaturrecherche konnte festgestellt werden, dass seit kurzer Zeit die Erforschung von Lernumgebungen an Bedeutung gewonnen hat und Begriffe wie „Der Raum als dritter Pädagoge“ gängig geworden sind. Margret Fell (2015) beschreibt Raumdidaktik als Planung und Gestaltung von materiellen Räumen, die nach pädagogisch-andragogischen Ansprüchen physikalisch, ästhetisch, funktional und extrafunktional so zu arrangieren sind, dass sie auf Bildungsprozesse einen förderlichen Einfluss haben. Sie argumentiert, dass Bildungsräume als didaktische Stützfunktion wirken, sofern diese günstige Konstellationen schaffen, die für eine am selbstgesteuerten Lernen orientierte Weiterbildung ermöglichen. Räume unterstützen auf diese Weise gezielt didaktische Grundformen, indem sie beispielsweise Orte für Begegnung und Dialog anbieten.

Es wird deutlich, dass der Raum als dritter Pädagoge wirksam wird und sich Raum und Lernende gegenseitig beeinflussen. Die Erarbeitung von Inhalten findet wie bereits in Abschnitt 2.3.1 hergeleitet, nicht isoliert von der jeweiligen Umwelt statt. Es macht einen Unterschied, ob man z. B. im Garten sitzt und lernt oder in einem Büroraum. Erkenntnisse aus der Cognitive Science (vgl. Abschnitt 2.1.2.1) zeigen, dass die Ver- und Erarbeitung von Wissen in der Interaktion mit dem jeweiligen individuellen Umfeld stattfindet. Fell (2015) stellt dazu fest:

„Je unterschiedlicher Raumqualitäten sind, die ein Individuum erleben kann, desto umfassender gestaltet sich dessen kognitives Begreifen."

Vielfalt der Raumqualität unterstützt also kognitive Prozesse. Dies bedeutet auch, dass nicht gut gestaltete Lernumgebungen negative Einflüsse auf den Lernerfolg haben. Sie führen zu schlechterer Motivation, mangelndem Wohlbefinden und Lernängsten (vgl. ebenda, S. 50).

Der Prozess der gegenseitigen Beeinflussung von Mensch und Raum ist für beide Richtungen gültig. Beeinflusst der Raum den Menschen und gibt Handlungsimpulse vor, so strukturiert der Mensch den Raum z. B. durch das Gruppieren von Möbeln. Die so entstehenden neuen Gegebenheiten des Raumes ermöglichen wiederum neue Handlungen. Auch der Autor Mehrabian (1987) weist darauf hin, wie wichtig eine anregende, ,lustbetonte“ Lernumgebung für den Lernerfolg ist. 
Allerdings gibt es hierzu wenig empirisch abgesicherte Befunde, die einen direkten Zusammenhang zwischen Verhalten und Raum nachweisen. Unterschiedliche Disziplinen wie Bildungsforschung, Architektur, Raumsoziologie, Pädagogik und Psychologie nähern sich diesem Feld derzeit überwiegend auf theoretischer Basis. Eine neuere Studie von Wieber und Conrad (2015) hat zum Ziel, Lernräume aus umweltpsychologischer Perspektive zu erforschen und Gestaltungsmerkmale und ihre Wirkung auf das Lernen zu identifizieren. Die Ergebnisse sind derzeit jedoch noch nicht veröffentlicht ${ }^{13}$.

An der Hochschule der Medien (HdM) werden Lernräume im Forschungsschwerpunkt „Lernwelten“ unter der Leitung von Prof. Richard Stang seit 2008 intensiv beforscht. Mit dem Ende 2011 eröffneten LearnerLab (vgl. Abbildung 2.8) steht seit mehreren Jahren ein geeignetes Untersuchungsumfeld für die gezielte Lernraumforschung zur Verfügung. Im Rahmen der Forschungsarbeiten wurde zunächst über mehrere Semester eine apparative Beobachtung des LearnerLabs mit Hilfe zweier Kameras, die alle 15 Minuten ein Bild des Raumes aufnahmen, durchgeführt. Apparative Beobachtungen, die automatisiert ablaufen, haben den Vorteil, dass Messfehler oder auch Fehler, die durch den Faktor „Mensch“ entstehen können, von vornherein ausgeschlossen werden können.
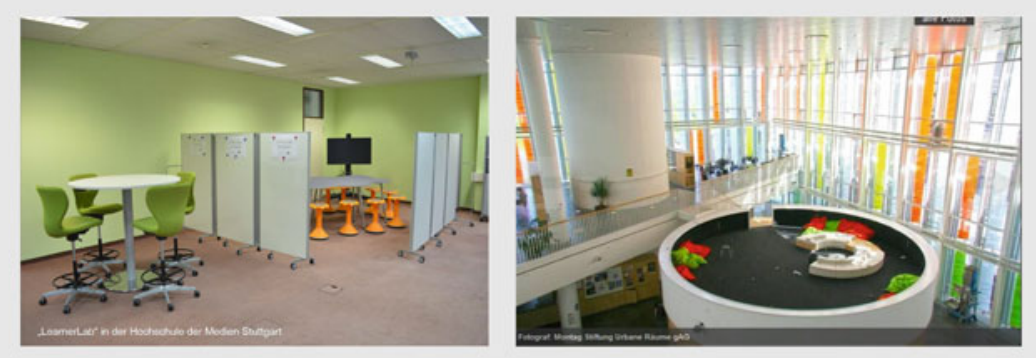

Abbildung 2.8 LearnerLab HdM Stuttgart (Quelle: HdM) und Orestad Gymnasium (Quelle: Montag Stiftung)

Empirisch untersucht wurde die Nutzungshäufigkeit, die Sozialform beim Lernen, die Arbeitsdauer und die Lern- und Arbeitsplatzwahl (Stang \& Strahl, 2016). Die Befunde zeigen, dass die Möglichkeit zur Schaffung eines eigenen Territoriums (Raum-im-Raum Prinzip) einen hohen Stellenwert für die Nutzer*innen

\footnotetext{
${ }^{13}$ Stand: 09.10.2017
} 
hat. Somit bestätigte sich die These von Gustave Nicolas Fischer, die besagt, dass sich verschiedene Aneignungsformen zu strategischen Verhaltensweisen verbinden und so die emotionale und soziale Anpassung an die jeweilige Aufgabe ausdrücken (Fischer, 1990). Zur Abschottung wurden überwiegend flexible Stellwände genutzt. Es konnte zudem festgestellt werden, dass vor allem die Ecken eines Raumes zum Lernen aufgesucht werden, so dass ausreichend Abstand zu anderen „Lerngruppen“ vorhanden war. Die Studie konnte empirisch nachweisen, dass die selbstgesteuerte Gestaltung und Anpassung des Raumes auf eigene Bedürfnisse positiv bewertet wird. Entsprechend sollten Lernräume über flexible Strukturen und Raumelemente verfügen. Darüber hinaus zeigte sich, dass sich große Flächen besser für Zonenkonzepte eignen (vgl. ebenda).

Die Montag Stiftung trägt seit 1998 Gestaltungsleitlinien für eine Pädagogische Architektur zusammen, indem sie Best-Practice Beispiele aus Europa identifiziert und auf ihrer Homepage vorstellt. Dabei fokussiert sich die Montag Stiftung insbesondere auf Schulbauten. Da aber auch im schulischen Bildungssystem modernere Lernformen im Zentrum aktueller Debatten stehen, sollen im Folgenden die von der Montag Stiftung ausgearbeiteten Leitlinien und Prinzipien vorgestellt werden, da sich hier grundlegende Muster erkennen lassen, die sich auch auf arbeitsplatzbezogene Lernformen übertragen lassen. In einem sich anschließenden Schritt erfolgt dann eine gesonderte Betrachtung von Gestaltungsprinzipien einer Büroarchitektur.

Zunächst einmal plädiert die Montag Stiftung auch für den schulischen Kontext auf eine ausgeprägt kompetenzorientierte Lernmethodik. Danach ist der Erwerb von Kompetenzen ein Lernprozess, in dem Aktivitäten, Emotionen, Kognitionen und Situationen auf vielfältige Weise miteinander verknüpft sind. Wenn dieser Lernprozess aktiv und ergebnisorientiert gestaltet ist und unterschiedliche Zugänge zum Lernen ermöglicht, ist er besonders effektiv (Bahner \& Montag Stiftung Jugend und Gesellschaft, 2017).

Hierbei ist festzustellen, dass die hier eingenommene lerntheoretische Grundlage mit den für die Forschungsarbeit relevanten arbeitsplatzbezogenen Lernprinzipien (vgl. Abschnitt 2.1.2) übereinstimmt und auch kompatibel mit den theoretischen Grundlagen zur Dekonstruktion des Begriffes Lernraum ist (vgl. Abschnitt 2.3.1). Insofern ist eine nähere Erläuterung der Gestaltungsprinzipien der Montag Stiftung auch für die vorliegende Forschungsarbeit relevant, obwohl sich diese explizit an die Gestaltung von Schulen richten. Grundlage der folgenden Ausformulierungen ist ein Verständnis, in dem Lernen als aktiver und interaktiver Prozess begriffen wird und in Folge dessen ein anderes als das bisher übliche Raumangebot benötigt. Als „Häuser des Lernens“ entwickeln sich Schulgebäude zu einer vielfältig nutzbaren Infrastruktur, die unterschiedlichen 
Lehr- und Lernumgebungen Raum gibt. Neben Räumen, die primär dem Frontalunterricht dienen, und spezialisierten Bereichen wie Werkstätten, Labors und Ateliers sind unterschiedlich dimensionierte Arbeitsumgebungen für Teams erforderlich. Gute Lern- und Arbeitsumgebungen entstehen dabei erst, wenn sie einer erkennbaren pädagogisch-architektonischen Konzeption folgen und die erforderliche Vielseitigkeit mit weiteren Eigenschaften verbunden ist, die für Schulbauten unverzichtbar sind. Die nachfolgenden Kriterien formulieren wichtige Prinzipien und Qualitäten von Schulgebäuden.

Die thesenförmig zusammengetragenen Prinzipien einer pädagogischen Architektur aus dem Jahr 2012 lauten wie folgt (Montag Stiftung Jungend und Gesellschaft, 2012, S. 252-280):

These 1:

Lernen benötigt viele und unterschiedliche Perspektiven, Zugänge und Ergebnisse

These 2:

Gelernt wird allein, zu zweit, in der Kleingruppe, mit dem ganzen Jahrgang, jahrgangsübergreifend und auch im Klassenverband

These 3:

Ganztagsschule heißt Lernen, Bewegen, Spielen, Toben, Verweilen, Reden, Essen und vieles mehr - in einem gesunden Rhythmus

These 4:

Schulbuch und Kreidetafel werden ergänzt durch Tablet-PC, Smartboard und andere Neue Medien

These 5:

Förderung in einer inklusiven Schule geschieht in heterogenen Gruppen

These 6:

Kulturelles und ästhetisches Lernen muss durch Pädagogik und Architektur vermittelt werden

These 7:

Lernen in Gesundheit und Bewegung findet in anregender und weiträumiger Umgebung statt

These 8:

Demokratisches Lernen benötigt eine demokratische Schule

These 9: 
Schule ist im Umgang mit Umwelt und Technik ein Vorbild

These 10:

Die Schule öffnet sich zur Stadt - die Stadt öffnet sich zur Schule

In diesen Thesen, die Gestaltungsprinzipien der Lernräume implizieren, lassen sich etliche Analogien und Überschneidungen zum Lernen am Arbeitsplatz ableiten. Da diese Thesen in 2017 überarbeitet und innerhalb von sechs Gestaltungsprinzipien komprimiert wurden, soll im Folgenden auf diese Prinzipien differenzierter eingegangen werden (Bahner \& Montag Stiftung Jugend und Gesellschaft, 2017, S. 14-17):

\section{Prinzip: Pädagogisch-architektonische Grundkonzeption}

Wesentliche Voraussetzung für ein gutes Schulgebäude ist die architektonische Gesamtkonzeption, die ausgehend von einer pädagogischen Leitidee ein differenziertes und in sich schlüssiges Raumprogramm beinhaltet. Raumbildung und Gestaltung sollten klaren Prinzipien folgen, die sowohl in den verschiedenen Funktionsbereichen innerhalb und außerhalb des Gebäudes als auch beim Erscheinungsbild und der Einbindung in die Umgebung erkennbar sind. Schulen sind dabei Lern- und Lebensorte gleichermaßen (Abbildung 2.9).
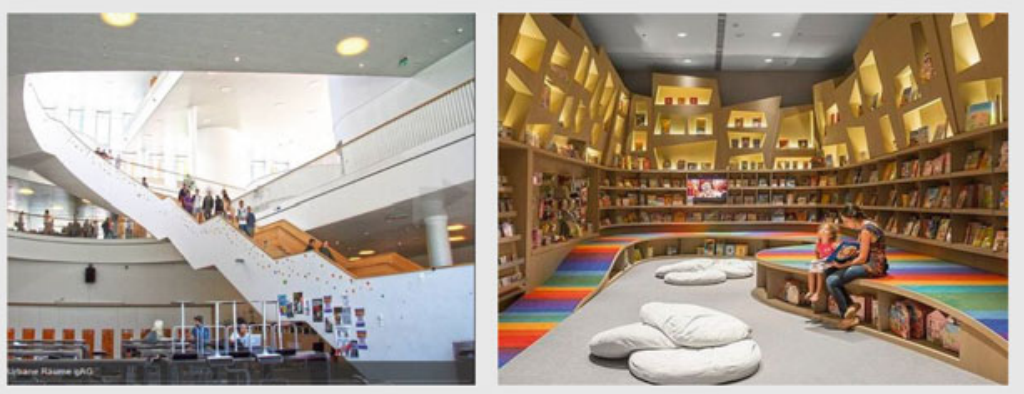

Abbildung 2.9 v.l.n.r.: Orestad Gymnasium (Quelle: Montag Stiftung) und Bibliothek Sao Paulo (Quelle: knstrct.com)

Die Grenzen verschwimmen hier ebenso wie beim arbeitsplatzbezogenen Lernen, wo Arbeiten, Lernen und Privatleben immer weiter miteinander verschmelzen. Bereits die Schulen müssen die Wissensarbeiter der Zukunft durch 
neue Bildungskonzepte unterstützen, wobei die Bildungsstrukturen in ähnlichen Mustern und Strukturen des lebenslangen Lernens am Arbeitsplatz verlaufen (Montag Stiftung Jugend und Gesellschaft, o. J.) (Abbildung 2.10).

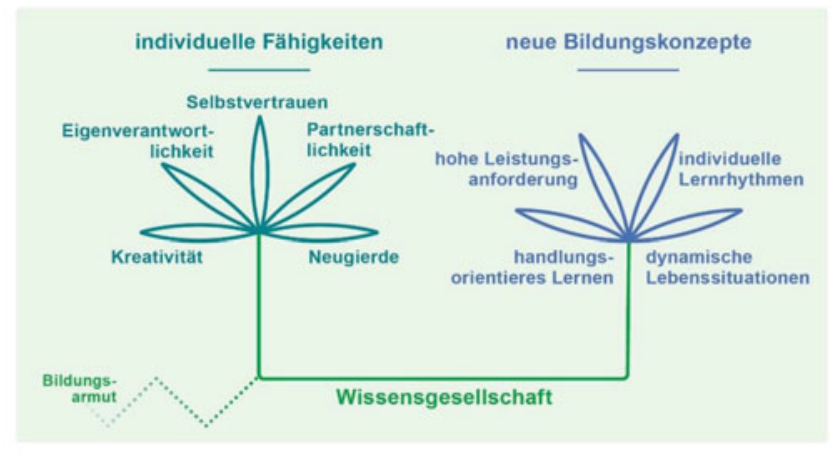

Abbildung 2.10 Die Wissensgesellschaft erfordert neue Bildungskonzepte (Quelle: Nach Montag Stiftung)

Die für eine pädagogisch-architektonische Grundkonzeption wichtigen Funktionsbereiche orientieren sich nach der Art der Lernaktivität (vgl. Abschnitt 2.1.2). Konkrete Tätigkeiten des Lernens sind z. B. die folgenden:

- Erkennen

- Abwägen

- Gestalten

- Entdecken

- Erörtern

- Planen

- Befragen

- Hinterfragen

- Bewerten

- Erleben

- Recherchieren

- Experimentieren 


\section{- Präsentieren}

Erfahrungsbasierte Lernkonzepte spiegeln sich in den o. a. Lernaktivitäten wider, die John Dewey (1859-1952) in einem Zitat wie folgt pointiert:

„Ein Gramm Erfahrung ist besser als eine Tonne Theorie.“

Entsprechend müssen die Lernräume o. a. Lernaktivitäten bzw. konkrete Lerntätigkeiten am Arbeitsplatz ermöglichen und durch die Raumgestaltung aktiv gefördert werden. Dabei sollte zunächst eine konkrete Bedarfs- und Kontextanalyse durchgeführt und auf den in Abschnitt 2.1.2.1. extrahierten förderlichen Lehrund Lernprozessen aufgebaut werden.

\section{Prinzip: Orientierung, Atmosphäre und Gestaltung}

Leistungsfähige Schulen zeichnen sich dadurch aus, dass sich die Lehrenden und Lernenden wohl fühlen. Dieses Wohlbefinden resultiert aus funktionalen, sozialen und ästhetischen Qualitäten eines Schulgebäudes. Dazu gehören die Zonierung in überschaubare Einheiten, klare räumliche Strukturen und Wegeführungen, prägnante Raumatmosphären und Sichtbeziehungen innerhalb und außerhalb des Schulgebäudes sowie eine sorgfältig ausgewählte Material-, Licht- und Farbgestaltung. Gut gestaltete Schulgebäude sind ein Ausdruck der Wertschätzung, die eine Gesellschaft der Bildung und ihren Akteurinnen und Akteuren entgegenbringt. In diesem Prinzip spiegeln sich die raumtheoretischen Grundlagen, wie sie von Sesink (2007) oder auch Bollnow (2010) im Sinne eines erlebten, subjektorientierten Raumes formuliert wurden (vgl. Abschnitt 2.3.1) wider. Entsprechend müssen harmonische Formen, Konturen, Strukturen und Lichtverhältnisse gestaltet werden, die eine angenehme Raumatmosphäre erzeugen.

3. Prinzip: Vielseitigkeit und Veränderbarkeit:

Die Veränderung von qualitativen und quantitativen Raumbedarfen sowie die Notwendigkeit, pädagogische und schulorganisatorische Konzepte fortwährend weiterzuentwickeln, macht die Anpassungsfähigkeit von Schulgebäuden zu einer grundlegenden Anforderung.

Dies betrifft sowohl die kurzfristige (situative) wie auch langfristige (konzeptionelle) Veränderbarkeit und gilt für unterschiedliche Maßstabsebenen vom einzelnen Lern- und Arbeitsraum über die gemeinschaftlichen Bereiche bis zum ganzen Gebäude. Die Räume sollten für unterschiedliche Aktivitäten nutzbar, einzelne Teilbereiche nach Bedarf miteinander kombinierbar und auch die Erschließungszonen eines Gebäudes für Lern-, Arbeits- und Erholungsphasen 
aktivierbar sein. Aber nicht der Verzicht auf spezifische Raumqualitäten zugunsten größtmöglicher Neutralität, sondern die Bereitstellung eines vielschichtigen Raumangebots schafft die dafür notwendigen Voraussetzungen.

Diesem Prinzip folgend sollten möglichst flexible Raumelemente verwendet werden, die sich schnell und einfach an den Bedürfnissen der jeweiligen Lernsituation anpassen lassen und wenn möglich nicht fest installiert sind.

4. Prinzip: Langlebigkeit und Wirtschaftlichkeit

Die Langlebigkeit von Schulgebäuden lässt sich auf verschiedene Weisen sichern: durch den Einsatz wertbeständiger und alterungsfähiger Materialien, kontinuierliche Pflege und Instandhaltung der Gebäude sowie einfacher Reparierbarkeit ihrer technischen Systeme, Vielseitigkeit und Veränderbarkeit ihrer Funktionsbereiche, aber auch durch eine besondere gestalterische Qualität ihrer Architektur, die mit der damit verbundenen öffentlichen Wertschätzung dauerhafte Erhaltungsinvestitionen sicherzustellen hilft. Ein langfristiges Grundelement für Dauerhaftigkeit ist vor allem die innere Flexibilität und Wandelbarkeit, denn äußerliche Veränderungen in der Gebäudestruktur lassen sich nur ungleich schwerer umsetzen.

Langlebigkeit und Wirtschaftlichkeit sind einander ergänzende Qualitätsziele. Neben den Erstellungs- und Einrichtungskosten eines Gebäudes sind in besonderem Maße die Kosten für Bewirtschaftung und Instandhaltung (Lebenszykluskosten), der Grundsatz der Umweltverträglichkeit und die größtmögliche Ressourcenschonung im Sinne nachhaltigen Bauens zu berücksichtigen. Zur Minimierung des Energieverbrauchs empfiehlt sich ein integriertes Gebäudekonzept mit einem ausgewogenen Verhältnis aus klugen Raumlösungen, sorgfältig ausgewählten Materialien und intelligenter Gebäudetechnik. Vorausschauende, mitunter höhere Anfangsinvestitionen machen Schulgebäude im Lebenszyklus wirtschaftlicher und wertbeständiger.

In diesem 4. Prinzip werden die Potenziale intelligenter und hybrider Lernräume durch den Einsatz von IoT-Lösungen geradezu von der Montag Stiftung eingefordert und indirekt benannt, ohne jedoch näher auf das Thema Internet der Dinge und dessen Möglichkeiten einzugehen. Entsprechend sind energieeffiziente IoT-Lösungen (z. B. Smart Metering) zu integrieren, die automatisiert die Heizung, Beleuchtung sowie weitere Geräte ausschalten, sofern sie nicht mehr benötigt werden. Darüber hinaus sind Funktionalitäten für energieeffizientes Wirtschaften möglich, indem Sensoren und Aktoren zwischen Heizkörperthermostat und Fenstern dafür sorgen, dass die Heizung abgeschaltet wird, wenn die Fenster geöffnet werden. Dies ist nur ein Beispiel von einer Vielzahl an energiesparenden und nachhaltigen Lösungen, die durch das Internet der Dinge erzielt werden können. 


\section{Prinzip: Gesundheit und Sicherheit}

Gesunde und sichere Lern- und Arbeitsbedingungen sind grundlegende und in hohem Maße lernförderliche Qualitäten eines guten Schulgebäudes. Mit dem Übergang zur Ganztagsschule und den damit verbundenen längeren Aufenthaltszeiten in Schulgebäuden sind die diesbezüglichen Anforderungen noch gewachsen. Für die Regelungsbereiche Licht, Akustik, Raumluft, Raumklima, Baustoffe, Sicherheit, Brandschutz und Hygiene existieren zahlreiche technische Normen und Vorschriften, die in unregelmäßigen Abständen novelliert werden. Sie haben dabei nicht nur den jeweiligen Stand der Technik, sondern auch die veränderten funktionalen Anforderungen an Lernumgebungen zu berücksichtigen. Dies betrifft zum Beispiel raumakustische Erfordernisse oder die Regelungen zum baulichen Brandschutz. Sie müssen künftig an zeitgemäße Konzeptionen gegliederter oder offener Lernlandschaften mit differenzierten Arbeits- und Unterrichtsphasen angepasst werden. Mit vielfältigen Bewegungsräumen und Sportmöglichkeiten, ausreichend Pflege- und Betreuungsangeboten sowie attraktiven Erholungs- und Rückzugsbereichen sollen Schulen die räumlichen Voraussetzungen für einen gesunden Schulalltag bieten.

In diesem 5. Prinzip eröffnen sich Potenziale intelligent gesteuerter Verfahren, die ein ergonomisches Arbeiten am Arbeitsplatz unterstützen (vgl. Abschnitt 2.3.3). Darüber hinaus sind auch Sicherheits- Klima- und Brandschutzautomatismen mit IoT-Lösungen aus dem SmartHome-Bereich abbildbar und lassen sich in Lernräume sinnvoll integrieren.

\section{Prinzip: Einbindung im Stadtteil}

Leistungsfähige Schulen und ihre Gebäude sind wichtige Bausteine einer Stadt, einer Gemeinde oder eines Quartiers. Sie kooperieren mit anderen öffentlichen Einrichtungen, sind Teile von Bildungslandschaften und dienen als Zentren lokaler Gemeinschaften, wenn sie zum Beispiel in den Abendstunden oder in den Schulferien auch für außerschulische Zwecke zur Verfügung stehen. Für die Integration des Schulgebäudes in das Stadtgefüge sind verschiedene Aspekte von Bedeutung: die Lage des Schulstandorts im Hinblick auf die verkehrliche Erreichbarkeit (öffentliche Verkehrsmittel sowie Bring- und Holverkehr), die räumliche Nähe zu anderen öffentlichen Einrichtungen, die Verfügbarkeit von räumlichen Reserven für mögliche Erweiterungen, die Ansiedlung komplementärer Einrichtungen, die städtebauliche Integration in die Umgebung und die Ausgestaltung der Schnittstellen und Übergangsbereiche zum öffentlichen Raum. Die Zonierung des Außenareals sollte es ermöglichen, dass anliegende Plätze, Parks und öffentliche Einrichtungen auf geeignete Weise in das Schulleben einbezogen werden können. 
Prinzip 6 ist das einzige Gestaltungselement, das sich nicht direkt auf ein Lernen am Arbeitsplatz abbilden bzw. übertragen lässt. Indirekte Zusammenhänge lassen sich jedoch in der Art herstellen, dass auch Unternehmen in einer regionalen und gesellschaftlichen Struktur eingebettet sind, die es zu nutzen gilt, z. B. in Form von Recruiting Events, Meet Up's etc, die in den Räumlichkeiten der Organisation stattfinden und auf aktuelle regionale Gegebenheiten Bezug nehmen. Auf der anderen Seite eröffnen sich visionäre Potenziale in Richtung einer „Smart City“ - Gerade wenn es um ein ortsabhängiges, adaptives Lernen am Arbeitsplatz geht (vgl. Abschnitt 2.1.2.5), hört dieses beim Verlassen des Bürogebäudes nicht schlagartig auf, sondern verläuft in ubiquitären, hybriden Bahnen auch außerhalb des Arbeitsplatzes weiter. Insofern wären hier Lernprozesse denkbar, die die Inhalte aus der Arbeitswelt (respektive Schulwelt) in die Alltagswelt überführen. Demnach könnte ein Lernszenario wie folgt aussehen:

Das adaptive Lernsystem weiß, dass sich Mitarbeiter*in XY beruflich mit dem Thema kontextgebundener Lernmethoden beschäftigt. Auf dem Heimweg läuft der/die Mitarbeiter*in an einer Agentur vorbei, die eine Stadtführung mit Hilfe einer Augmented Reality App anbietet. Das Smartphone registriert diese Agentur mit ihren inhaltlichen Schwerpunkten und empfiehlt für das Wochenende, an einer kontextbezogenen, hybridisierten Lern-Tour durch den eigenen Wohnort teilzunehmen.

Aufbauend auf diesen Grundlagen lassen sich zusammenfassend und im Abgleich weiterer Literaturanalysen zur Lernraumforschung (Eigenbrod \& Stang 2014; Barrasch 2013; Knoll 1995; Sesink 2014; Stang 2014) die folgenden Gestaltungsprinzipien destillieren:

- Kreieren einer affektiven Raumatmosphäre

- Gestaltung offener Lernlandschaften mit Rückzugsorten in Form von mobilen „Raumzellen“

- Bereitstellung einer multifunktionalen Raumausstattung

- Unterstützung unterschiedlicher Lernformate durch funktionale Vielseitigkeit
o Frontalunterricht
o Gruppenarbeit
o Einzelarbeit
o Präsentation

- Berücksichtigung einer ressourcenschonenden Architektur (z. B. durch Upcycling, vgl. Abbildung 2.11) 
- Berücksichtigung einer kostenbewussten Gebäudebewirtschaftung (z. B. durch energieeffiziente SmartHome-Lösungen)

- Nutzung flexibler Raumelemente zwecks einfacher Umgestaltung, die an den Bedürfnissen der Lehrenden und Lernenden ausgerichtet ist

- Partizipative Co-Creation der Lernräume

- Berücksichtigung und Nutzung der Außenbereiche

- Herstellung optimaler Bedingungen in Bezug zur Beleuchtung (viel Tageslicht), Beschattung, Akustik, Luftqualität, Kühlung und Heizung

- Unterstützung von Gesundheit, Sicherheit und ergonomischen Prinzipien

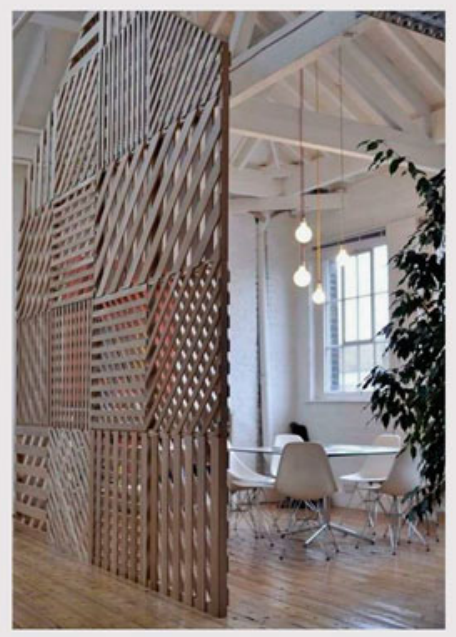

Abbildung 2.11 Upcycling mit Paletten als Trennwand (Quelle: Pinterest.de )

Die in diesem Abschnitt aufgeführten Bezüge zwischen Architektur und Pädagogik sollen nunmehr in einem abschließenden Schritt in den Kontext der eher wirtschaftswissenschaftlich geprägten Büroraumgestaltung gestellt werden. Ziel ist es, eine pädagogisch fundierte und ganzheitliche Perspektive auf die Gestaltung von Lernräumen mit Fokus auf arbeitsplatzbezogenes Lernen zu entwickeln. 
Zunächst einmal kann festgestellt werden, dass im Vergleich zu pädagogisch orientierten Untersuchungen umfangreichere Studien eher aus dem wirtschaftswissenschaftlichen Kontext vorangetrieben bzw. realisiert werden, die den Zusammenhang zwischen (Büro-)Räumen und dem Wohlbefinden der Mitarbeiter*innen sowie dem Mitarbeiterengagement, also der Produktivität der Angestellten, empirisch erforschen. Dies ist nicht weiter verwunderlich, da derartige Studien extrem aufwändig und sehr teuer sind und von daher nicht von Kommunen, öffentlichen Bildungsträgern oder gar den (Hoch-)Schulen selbst in Auftrag gegeben werden können. Die Institutionen, die derartige Studien durchführen, sind oftmals in wirtschaftliche Interessenlagen involviert, so dass die Untersuchungen kritisch zu reflektieren sind.

So enthält beispielsweise der Steelcase Global Report insgesamt fünft Erkenntnisse, die bei einer Gestaltung der Büroräume relevant sind (vgl. Abbildung 2.12).

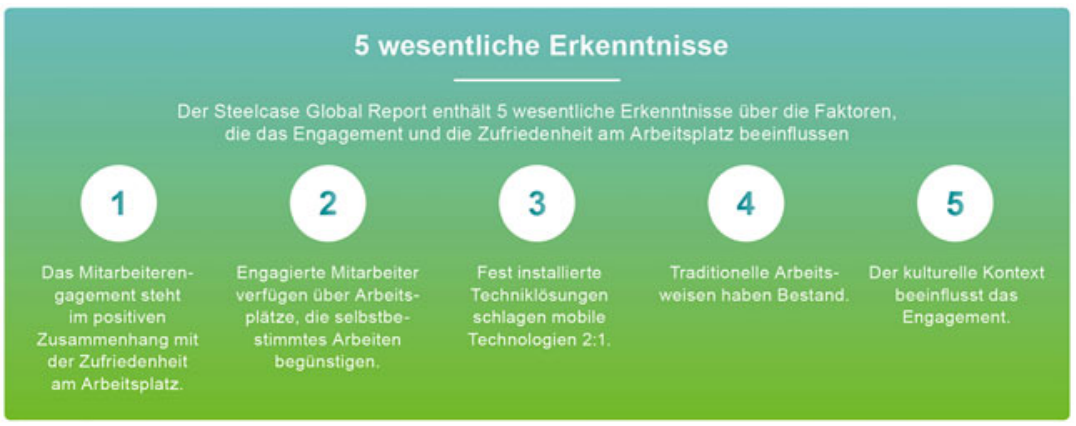

Abbildung 2.12 Faktoren, die das Engagement und die Zufriedenheit am Arbeitsplatz beeinflussen (Quelle: Nach Steelcase Global Report 2016)

In überwiegenden Teilen decken sich diese Erkenntnisse mit den Prinzipien pädagogischer Architektur der Montag Stiftung. Steelcase (2015) weist aber darüber hinaus darauf hin, dass ein weltweiter Trend zu erkennen sei, der sich in Form einer weltweiten kulturellen Bewegung definiere und den Arbeitsplatz gänzlich neu erfinde. Dazu gehöre auch der Trend der „Maker-Szene“. Steelcase formuliert dieses Phänomen wie folgt (vgl. ebenda, S. 1):

„Die „Maker-Szene“ - eine Art Do-it-yourself- Avantgarde, die leidenschaftlich gern Dinge schafft und Communitys bildet, um Gerätschaften, Räume und Ideen zu teilen - hat sich zu einem weltweiten Phänomen entwickelt. Maker-Messen, Maker-Bereiche 
und Maker-Magazine sind sichtbare Zeichen für etwas, das manche als „Do-okratie“ bezeichnen - eine Renaissance des Selbermachens, mit Menschen, die ihre Ressourcen teilen und sich in ihrer Kreativität gegenseitig unterstützen.“

Steelcase verweist in diesem Zusammenhang auf einen Wandel von einseitiger, überwiegend wirtschaftlich geprägter Effizienz hin zu vielfältigeren Lösungen, die das seelische, körperliche und kognitive Wohlbefinden der Menschen fördern. Angesichts der globalen Suche nach Talenten und der wachsenden Notwendigkeit für mehr Mitarbeiterengagement nehmen informelle, authentische und inspirierende Räume an Bedeutung zu.

Im letztgenannten Satz schwingt bereits die Ursache dieser zu beobachtenden Veränderungen mit, die darüber hinaus die bedeutungstheoretischen Annahmen über einen erlebten (vgl. Bollnow 2010) und poietischen (symbolischen) Raum (vgl. Sesink 2007) untermauern. Die Maker-Szene ist darüber hinaus begleitet durch eine Ausbreitung sogenannter Co-Working \& Creative-Spaces (Abbildung 2.13).

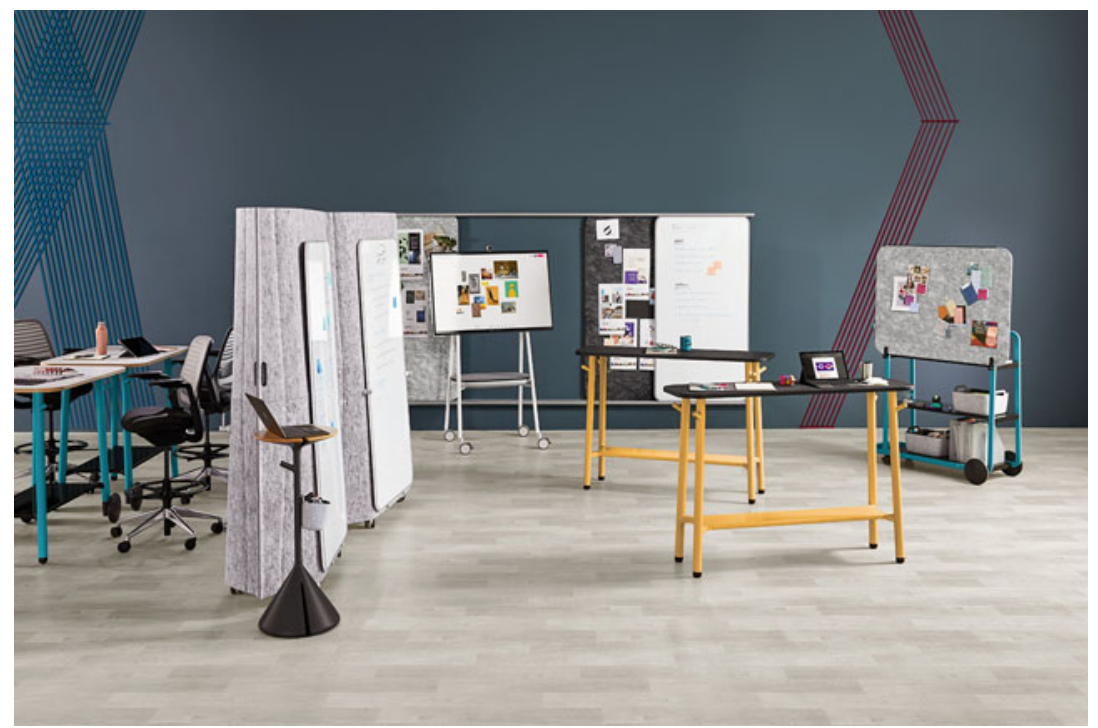

Abbildung 2.13 Formen, Farben und Struktur erzeugen Atmosphäre (Quelle: Steelcase ) 
Co-Working- Spaces (Abbildung 2.14) sind Bürogemeinschaften, die in der Regel einen Arbeitsplatz für Start-ups und Freiberufler anbieten. Derartige Bürogemeinschaften wie z. B. das Betahaus ${ }^{14}$ in Berlin haben sich längst weltweit etabliert. Der Trend kommt ursprünglich aus dem Silicon Valley, dem „Kreativtal der USA“, in dem die Unternehmen Google und Facebook ihre digitalen Produkte erarbeiten.

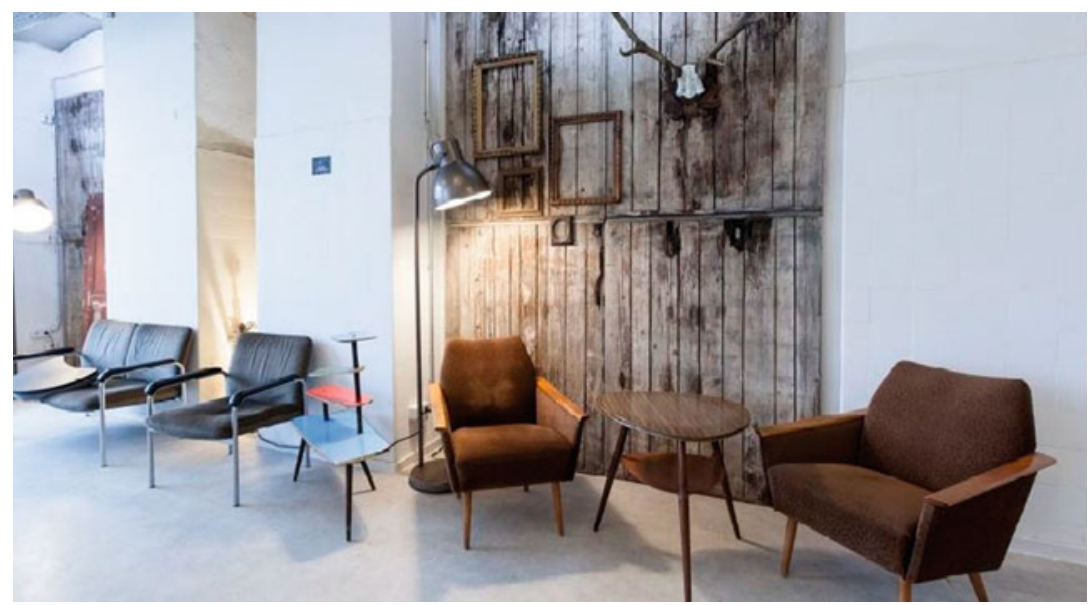

Abbildung 2.14 Co-Working Space (Quelle: Ahoy Berlin)

Kernkonzept sind offene Räume für offene Gedanken. Kreative und Freie jeder Art sollen eine Arbeitsatmosphäre vorfinden, die ihre Ideen zutage fördert (Abbildung 2.15). In diesem Sinne sind innovative Arbeitsmethoden an der Tagesordnung und die Räume gehen entsprechend auf diese neuen designbasierten Lern- und Arbeitsformen ein. Als Trendsetter im Zusammenhang designbasierter Lernmethoden wird oft auf die d.school Stanford ${ }^{15}$ verwiesen, die derartige Projekträume seit vielen Jahren entwickelt und im eigenen Lehrbetrieb einsetzt (Barrasch, 2013).

Zudem sollen in Co-Working-Spaces möglichst viele Menschen mit unterschiedlichen Berufen in einem Raum zusammenarbeiten, um neue Perspektiven auf ein Problem zu bündeln und innovative Lösungen zu kreieren. Eine derartige

\footnotetext{
${ }^{14}$ https://www.betahaus.com/berlin/

${ }^{15} \mathrm{https}: / /$ dschool.stanford.edu
} 


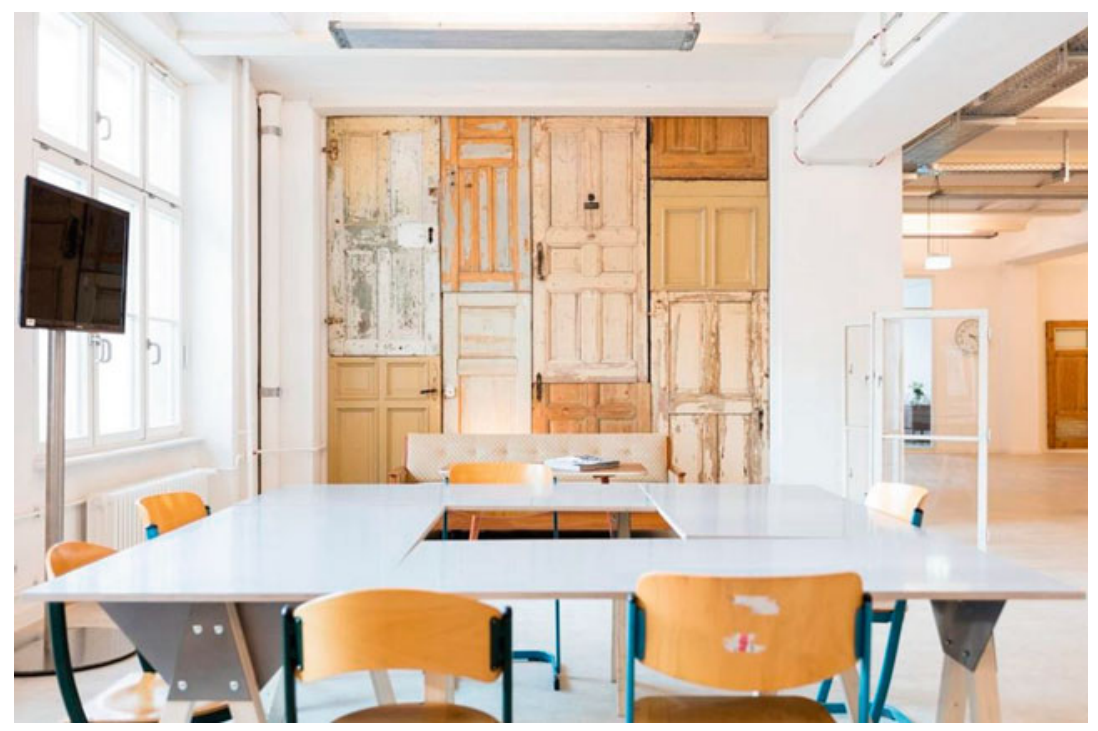

Abbildung 2.15 Co-Working Space (Quelle: Ahoy Berlin)

Arbeitsplatzgestaltung mit den damit verbundenen Arbeitsmethoden werden derzeit auch von großen, traditionellen und etablierten Unternehmen entdeckt, die die überaus kreativ gestalteten „Netzwerk-Oasen“ und Raumgestaltungselemente der öffentlichen Co-Working Spaces in den eigenen Bürolandschaften übernehmen wollen.

Denn auch hier ist das bekannte Zonen-Konzept (vgl. Montag Stiftung) längst angekommen, bei welchem unter dem Konzept des „Future Workplace“ unterschiedliche Büroraumbereiche separat zu gestalten sind.

So wurden beispielsweise auf der Future Workplace Tagung aus $2016^{16}$ folgende Kernbereiche für die Gestaltung der Microsoft Zentrale in München vorgestellt, die sich an den drei Handlungsfeldern (Mensch, Ort, Technologie) orientierten (Abbildung 2.16):

Besonders auffällig ist auch hier eine Kongruenz zwischen den zu gestaltenden Raumzonen im Vergleich zu den in Abschnitt 2.3.1 zugrunde gelegten theoretischen Bezügen, wobei sich der Raum in Wechselwirkung der drei

${ }^{16} \mathrm{Vgl}$. Konferenzband unter: https://bit.ly/2glvZcU 


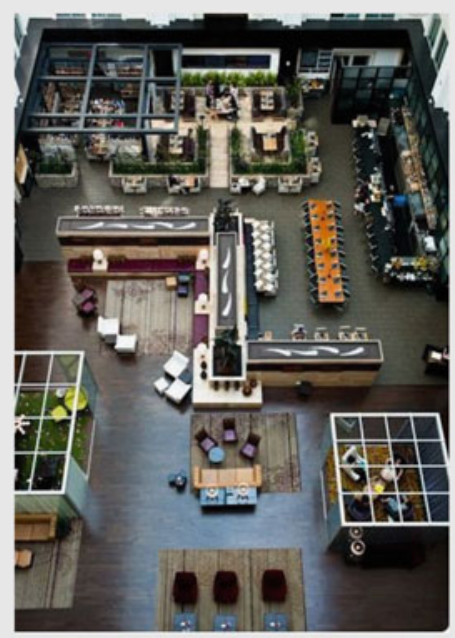

\section{Accomplish:}

Einzelarbeit für fokussierte Arbeitsweisen.

2. Think:

Einzelarbeit für ruhige Arbeitsweisen ohne Technologiebedarf.

\section{Share \& Discuss:}

Kollaboration für energiegeladene und informelle Arbeitsweisen.

\section{Converse:}

Kollaboration für offene, formelle und informelle Arbeitsweisen.

Abbildung 2.16 Zonen-Konzept (Quelle: kimberlytaylorimages.com)

Raumkomponenten (materiell (Ort), sozial (Mensch) und virtuell (Technologie) manifestiert.

Ein Bildungsforscher von Steelcase fasst die neue Wertigkeit physischer Büroräume wie folgt zusammen (Kim, 2015, S. 2):

„Die Maker-Szene zelebriert das Zusammenarbeiten ebenso wie den physischen Akt des Machens. Unsere Forschungen haben ergeben, dass zur gleichen Zeit, in der neue Technologien die Pädagogik verändern, auch die Bedeutung des Präsenzlernens zunimmt - Lehrer können dadurch die Möglichkeiten des praxisnahen Lernens einsetzen anstatt sich nur auf Vorlesungen zu fokussieren.“

Auf dieser Grundlage entwickelte Steelcase Gestaltungsprinzipien, die auf Grundlage eigener Forschungsarbeiten über kreative Lernorte (Abbildung 2.17) folgende Leitlinien zusammenfassen:

- Sorgen Sie für eine inspirierende Atmosphäre: Helle Farben, bequeme Sitzmöbel, Tageslicht und Blickbeziehungen ins Freie erhöhen die Lernbereitschaft und fördern das kreative Denken, während ausdruckslose Umgebungen zu Langeweile führen. 
- Ermöglichen Sie Flexibilität und Individualität: Ermöglichen Sie es Lehrenden und Lernenden, Räume nach individuellen Bedürfnissen neu zu konfigurieren - Anforderungen können sich dabei von Lernsetting zu Lernsetting, aber auch innerhalb einer Abteilung verändern. Eine mobile Möblierung ist zur Realisierung vielfältiger Raumkonfigurationen unerlässlich.

- Schaffen Sie unterschiedliche Zonen: Teamarbeit und schöpferische Prozesse sind oft laut, während es zur Kontemplation Ruhe bedarf. Vergewissern Sie sich, dass das Raumlayout und die Möbel so flexibel sind, dass sie auch Mitarbeiter*innen unterstützen, die zwischen den Teamsitzungen allein arbeiten wollen. Vor allem in Räumen mit Maschinen zum Prototypenbau müssen Denk- und Arbeitsbereiche so weit wie möglich entfernt liegen.

- Haben Sie keine Angst vor Unordnung: Das Ausbreiten von Unterlagen hilft Teams, Ideen und Möglichkeiten „laut zu denken“. Sorgen Sie für große Arbeitsflächen, die den Teams viel Platz bieten, aber auch für viel Stauraum z. B. für Arbeitsmittel und Arbeitsmodelle.

- Sehen Sie vertikale Präsentationsflächen vor: Was an oder auf einem Tisch passiert, ist nur für die unmittelbar Beteiligten sichtbar. Mit mobilen oder fest montierten Whiteboards können Ideen und erste Erfolge hingegen mit allen leicht geteilt werden. Whiteboards und Textmarker kann es eigentlich nie zu viele geben.

- Fördern Sie vielfältige Körperhaltungen: Die Körperhaltung sowie Körperbewegungen beeinflussen kreative Prozesse.

- Stehen in Gruppen kann zu mehr Interaktionen und einer erhöhten Aufmerksamkeit führen, während Einzelarbeit und das Entstehen neuer Denkweisen eher durch bequeme Sitzhaltungen oder das Herumlaufen gefördert wird.

- Sorgen Sie für einen einfachen digitalen Informationsaustausch: Relevante Inhalte liegen zunehmend in digitaler Form vor. Setzen Sie Techniken ein, die die Kommunikation zwischen den eingesetzten Geräten ebenso erleichtern wie den Gedankenaustausch.

Im Vergleich zu den einleitend dargestellten pädagogisch fundierten Gestaltungsprinzipien lassen sich ergänzende, spezifische auf den Arbeitsplatz bezogene Gestaltungsprinzipien aus einer wirtschaftswissenschaftlichen Perspektive herausarbeiten. Um ein ganzheitliches Bild über die Anforderungen einer pädagogisch fundierten, arbeitsplatzbezogenen Gestaltungsarbeit zu zeichnen, soll abschließend eine Zusammenfassung der wesentlichen Aspekte erfolgen (Abbildung 2.17). 

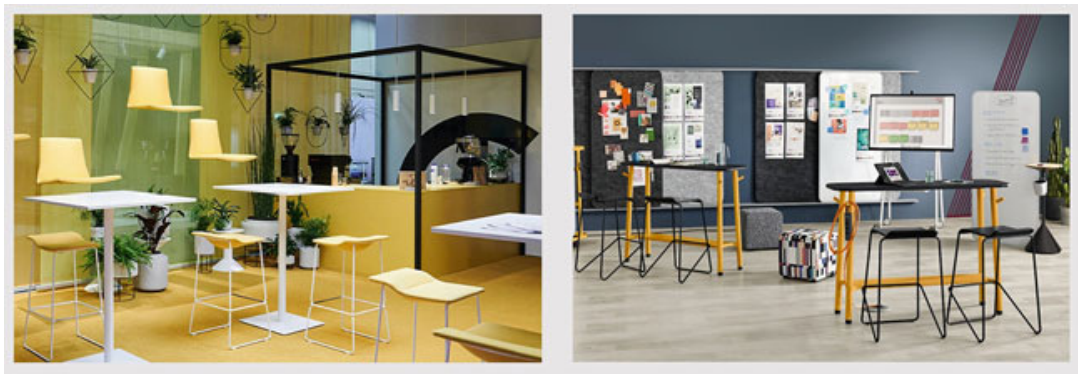

Abbildung 2.17 Offene und kreative Arbeitsbereiche (Quelle: Steelcase)

Die Lernräume sollten vor dem Hintergrund vielseitiger Lernmethoden auch flexibel nutzbar sein. Sowohl kreative Teamarbeiten, als auch konzentriertes Arbeiten in Rückzugsbereichen sollten gefördert werden.

Gleichzeitig sollte ausreichend Platz für Interaktionen, Dialoge und Präsentationen geboten werden. Für design- und innovationsbasierte Arbeitsweisen verwandeln sich klassische Schulungsräume zunehmend in „Experimentierwerkstätten und Forschungslabore“ (Maker-Spaces). Damit sich Arbeitnehmer*innen erproben können und ein erfahrungsorientiertes Lernen ermöglicht wird, brauchen sie ein Umfeld, in dem sich Ideen entwickeln und umsetzen lassen. Der Raum kann unterschiedliche Anforderungen an Lern- und Arbeitsprozessen unterstützen, indem er entsprechend gestaltet wird. Das Wechselspiel der Lernformen und Phasen des Lernens wird durch eine jeweils angepasste, flexibel nutzbare Raumgestaltung unterstützt. Dabei werden Raumbereiche für Einzelarbeit ebenso wie für kollaborative Teamarbeit zur Verfügung gestellt.

Das stimmungsvolle, affektive Unterstützen in den jeweils richtigen Modi kann durch eine bewusste, zielgerichtete und didaktisch fundierte Gestaltung der Räume ermöglicht werden. In einer Studie konnte der Zusammenhang der 5 Sinne mit einer positiven Bewertung der Lernenden nachgewiesen werden. Dabei konzipierten Architekten zwei völlig unterschiedliche Arbeitsumgebungen: einen neutralen und einen sinnlich gestalteten Raum. Als neutraler Raum diente ein Standard-Meetingraum mit weißem Besprechungstisch und gleichmäBiger Beleuchtung. Der sinnliche Raum hingegen war ausgestattet mit einem Tisch und (teilweise blau hinterleuchteten) Regalen aus duftendem, sägerauem Zedernholz, drei unterschiedlichen Design-Holzstühlen, einem mehrteiligen StoffSchiebevorhang sowie in den Regalböden ausgelegten Lebensmitteln (Steelcase, 2016b). 
Offene Lernlandschaften zeichnen sich durch ein reizvolles architektonisches Wechselspiel zwischen intimen Lernräumen und öffentlichen Erschließungszonen aus. Hier können sich Mitarbeiter*innen in konzentrierten Phasen zurückziehen (Abbildung 2.18). Gleichzeitig braucht es offene Räume, die den Austausch abteilungsübergreifend ermöglichen.

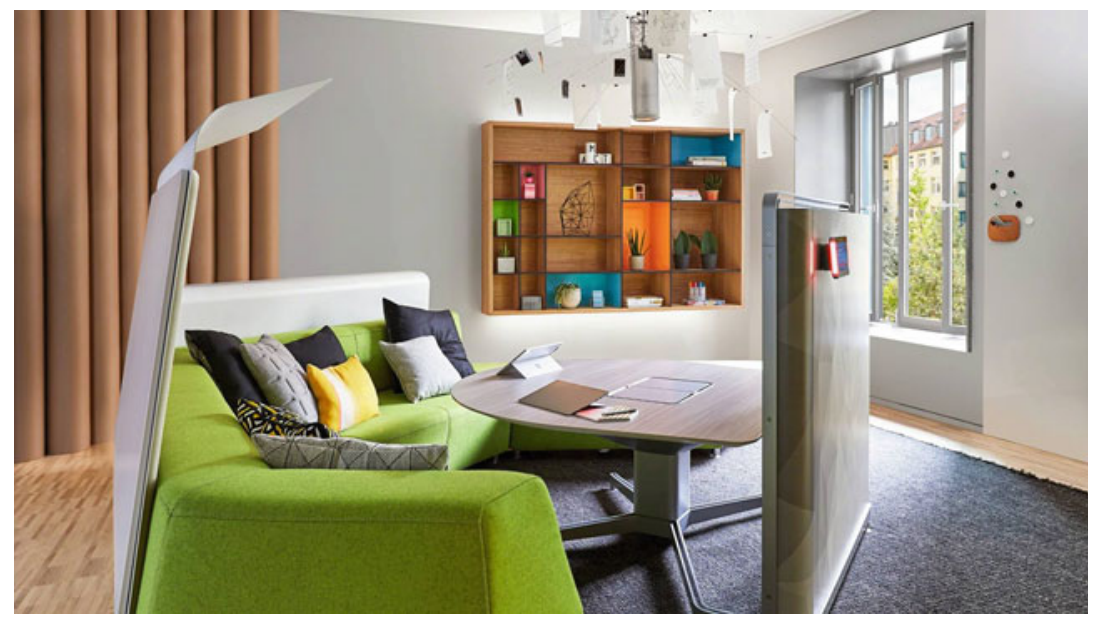

Abbildung 2.18 Kreative Rückzugsorte (Quelle: Steelcase)

Für kreative Phasen wiederum ist eine entspannte Atmosphäre wichtig. Der Raum kann mit Sitzkissen oder Sitzelementen ausgestattet sein, über beschreibbare Flächen, Whiteboards und Pinnwände verfügen, inspirierendes, kreatives Material sowie genügend Bewegungsfreiheit bieten. Doorley und Witthoft (2012) haben zusammen mit der d.school Stanford eine Handreichung zur Gestaltung von Kreativräumen erstellt, die speziell für kollaborative Zusammenarbeit spezifische Möbelstücke, Materialien und Tools empfiehlt.

Für Entscheidungsfindungen eignen sich Strukturen eines Meetingraums, in dem klare Rollen und Regeln definiert werden. Für die Anforderungen an den Raum, in dem Austausch und Kommunikation stattfinden, kann das „Wiener Café" mit seinen vielen Tischinseln inspirieren und zur Entspannung das gemütliche Wohnzimmer eine Orientierung bieten (Abbildung 2.19).

Bei offenen Bürolandschaften wird oftmals kritisiert, dass es an ausreichenden Ruhezonen fehlen würde. Während in bestimmten Phasen Austausch wichtig ist, 


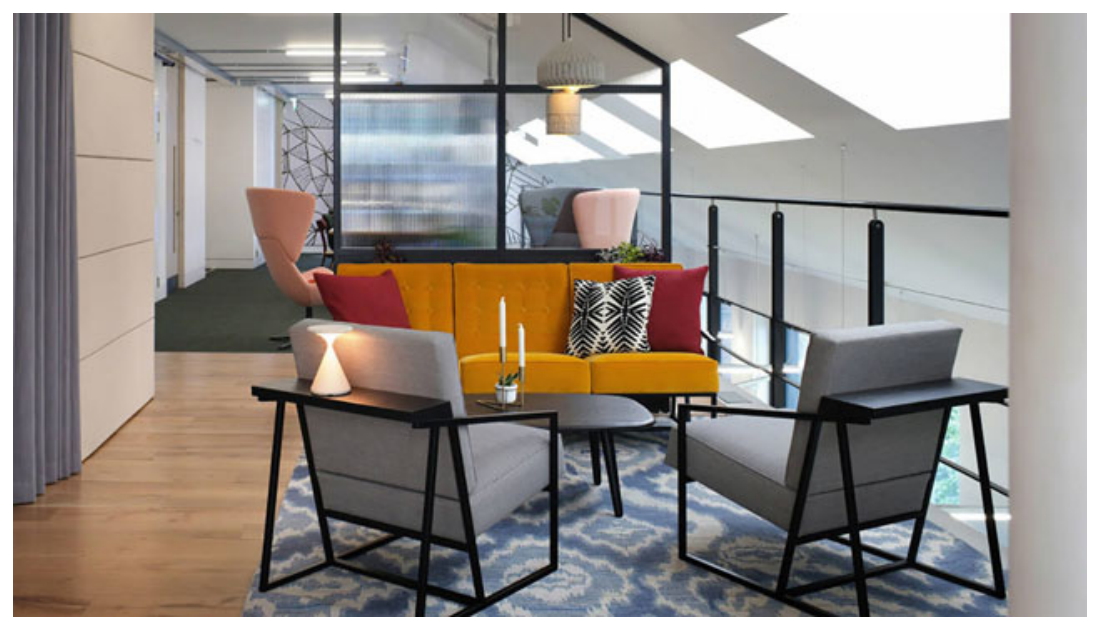

Abbildung 2.19 Gemütliche Cafe Atmosphäre (Quelle: Steelcase)

um sich Ideen mitzuteilen und Feedback zu holen, ist entsprechend in anderen Phasen genau das Gegenteil erforderlich: Ruhe und Konzentration. Die Ausstattung der Räume ist zurückhaltend, nicht schrill und laut. Sie unterstützt durch wenig Ablenkung ein konzentriertes Lernen und hilft dabei, die vielen Eindrücke $\mathrm{zu}$ verarbeiten und Ruhe einkehren zu lassen.

Im Gegensatz dazu gibt es „kollaborations-Bereiche“, in denen Fotos, Grafiken, Sketchnotes oder Post-it Notes mit visualisierten Ideen und Zeichnungen dominieren. Diese Räume können Gruppen schnell in einen gewünschten Projektmodus befördern, der innovatives Problemlösen unterstützt (Abbildung 2.20).

Trotz einer deutlichen Hinwendung auf kreative und kompetenzorientierte Lern- und Arbeitsweisen muss auch die Möglichkeit einer Wissensvermittlung weiterhin gegeben sein. Das Format des Frontalunterrichts wird weiterhin relevant sein und benötigt eine entsprechende räumliche Berücksichtigung. Darüber hinaus ist die Lernmethode „Lernen durch Lehren“ bzw. die Präsentation von Gruppenarbeiten ein wichtiges Element einer kompetenzorientierten Weiterbildung, so dass eine Art Plenum bei der Raumgestaltung integriert werden sollte (Abbildung 2.21).

Die eben skizzierten unterschiedlichen Raumarrangements unterstützten die Vielfalt unterschiedlicher Lernmethoden (Abbildung 2.22), wobei die spezifisch gestalteten Raumarrangements nicht zwangsläufig in unterschiedlich getrennten 

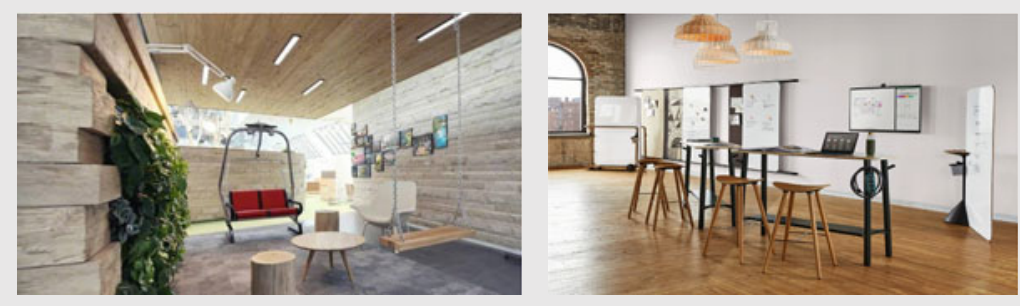

Abbildung 2.20 Förderung der Zusammenarbeit in Projekten (Quelle: Steelcase )

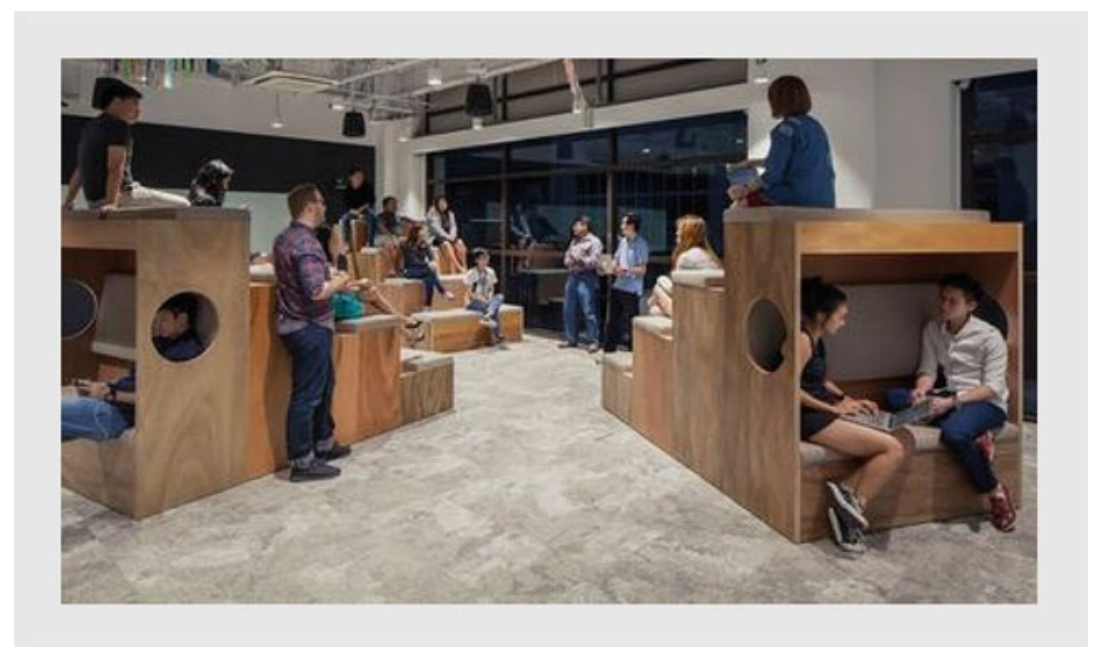

Abbildung 2.21 Airbnb-Zentrale (Quelle: Airbnb)

Räumlichkeiten realisiert werden müssen. Vielmehr geht es darum (v. a. bei Platzmangel) eine bewusste Inn,eneinrichtung zusammenzustellen, die auch in nur einem Raum unterschiedliche Lernszenarios umsetzen lassen (Abbildung 2.23).

In modern gestalteten Arbeitsumgebungen wird besonders deutlich, welchen Stellenwert die Beleuchtung hat. Zudem sind natürliche Materialien wie z. B. Natursteine, Holzelemente in Kombination mit Pflanzen sehr beliebt, um eine 


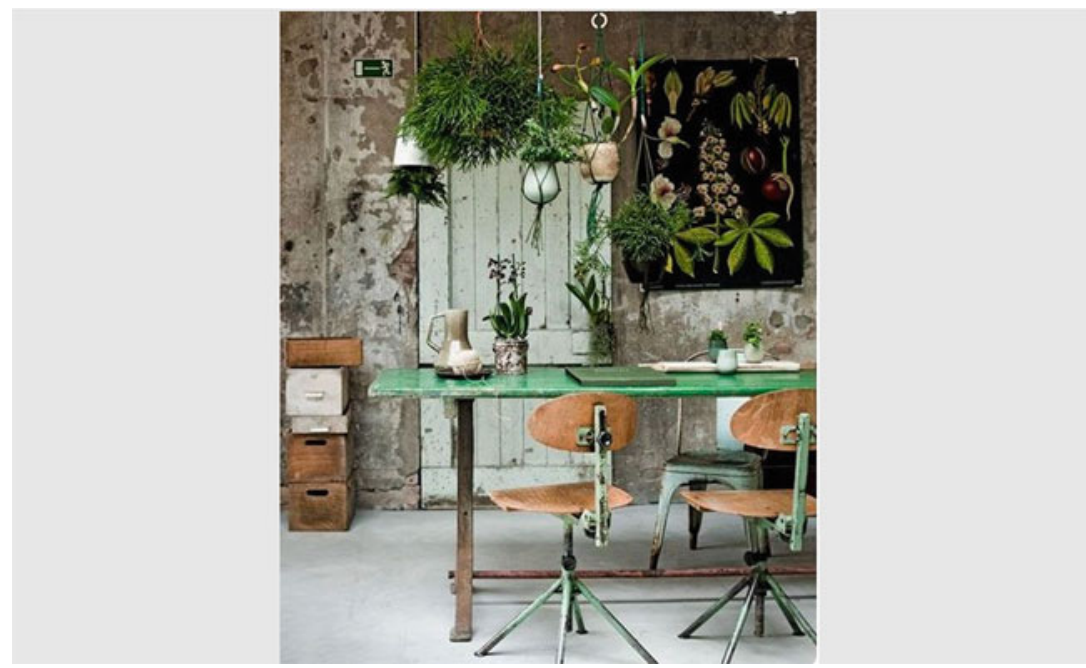

Abbildung 2.22 Naturnahe Gestaltung (Quelle: Best of Office Design, 2009)

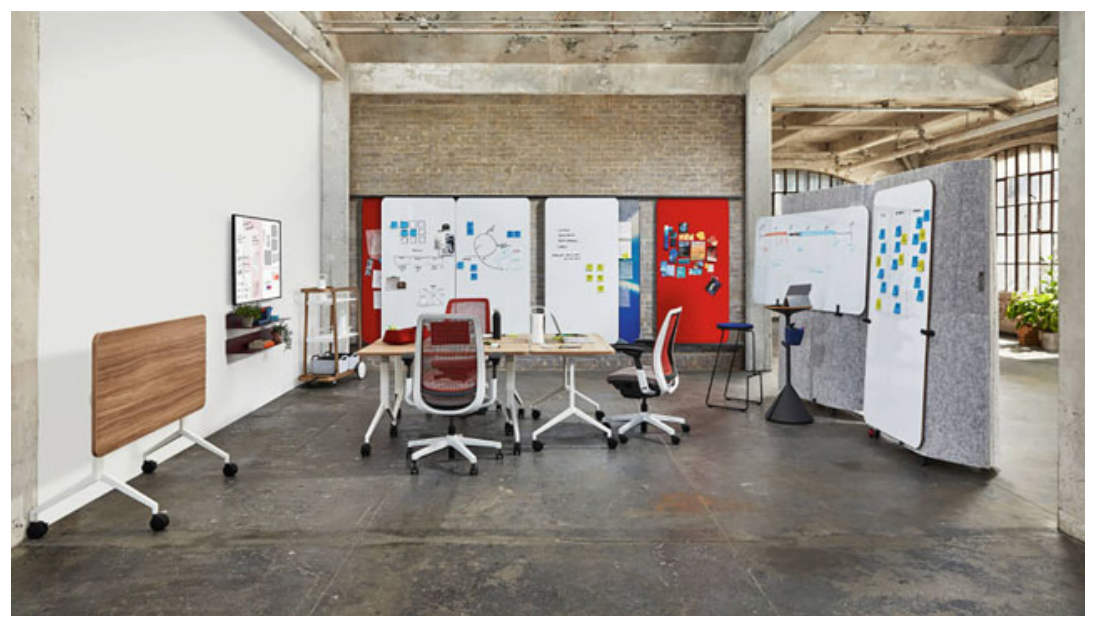

Abbildung 2.23 Unterschiedliche Szenarien ermöglichen durch offene Gestaltung (Quelle: Steelcase) 
angenehme Atmosphäre zu erzeugen. Zudem kann festgestellt werden, dass Bürolandschaften zunehmend im „Wohnzimmer-Style“ gestaltet werden verbunden mit dem Ziel, dass sich die Menschen dort gerne aufhalten. Ein Zugang zu Außenbereichen, in denen man umgeben von Natur lernen und arbeiten kann, haben zudem an Bedeutung gewonnen (Schulz, 2009).

\subsubsection{Intelligente und hybride Lernräume}

Ein hybrider Raum bzw. ein „Blended Space“ entsteht, wenn z. B. digitale und physische Bereiche eng miteinander verbunden werden und sich überlagern. Das Ergebnis dieser Vermischung ist ein neuer Bereich, der ganz eigene, neue emergente Eigenschaften besitzt und somit mehr ist als die Summe der Einzelteile (Kohls \& Münster, 2017).

Die in den vorhergehenden Abschnitten beschriebenen Lernräume werden $\mathrm{zu}$ intelligenten und hybriden Lernräumen transformiert, indem sie über eine integrierte, unsichtbare Technologie verfügen. Wie in Abschnitt 2.2 dargelegt, können jegliche Ausstattungs- und Lerngegenstände mit IoT-Komponenten angereichert werden. Die dadurch entstehenden Smart Objects werden wiederum untereinander und mit dem Internet vernetzt, so dass diese in einem komplexen Cyber-physischen System interagieren. Wenn auf diese Weise Lerngegenstände und Lernprozesse digitalisiert und vernetzt sind, entstehen bisher ungeahnte, didaktische Handlungsoptionen.

Ein Vorzeigeobjekt in Sachen nachhaltiger Büroarchitektur befindet sich in Amsterdam. Das Deloitte Headquarter ist ein Hightech-Büro und nennt sich „The Edge“. Es gilt nicht nur als das nachhaltigste Bürogebäude der Welt, sondern ist auch vollvernetzt und mit ca. 20.000 Sensoren ausgestattet (Abbildung 2.24).

Damit zählt das vom niederländischen Immobilienentwickler OVG Real Estate für die Wirtschaftsprüfungs- und Beratungsgesellschaft Deloitte erbaute „The Edge" zu den fortschrittlichsten Bürogebäuden der Welt. In dem 2015 eröffneten Hightech-Komplex ist alles auf Nachhaltigkeit ausgerichtet. Das 40.000 Quadratmeter große Bürogebäude produziert dank innovativer Technologien mehr Energie als die 1000 Deloitte-Angestellten verbrauchen können.

Die Südseite des Gebäudes ist mit hocheffizienten Sonnenpaneelen ausgestattet, sodass Sonnenstrahlen dann aufgefangen werden, wenn sie am stärksten sind. Außerdem ging OVG Real Estate eine Partnerschaft mit der Universität von Amsterdam (UVA) und der Hogeschool van Amsterdam (HVA) ein, um Energieneutralität zu erreichen. Mithilfe eines thermischen Aquiferspeichers in einer Tiefe von etwa 130 Metern unterhalb des Gebäudes wird ausreichend Energie 


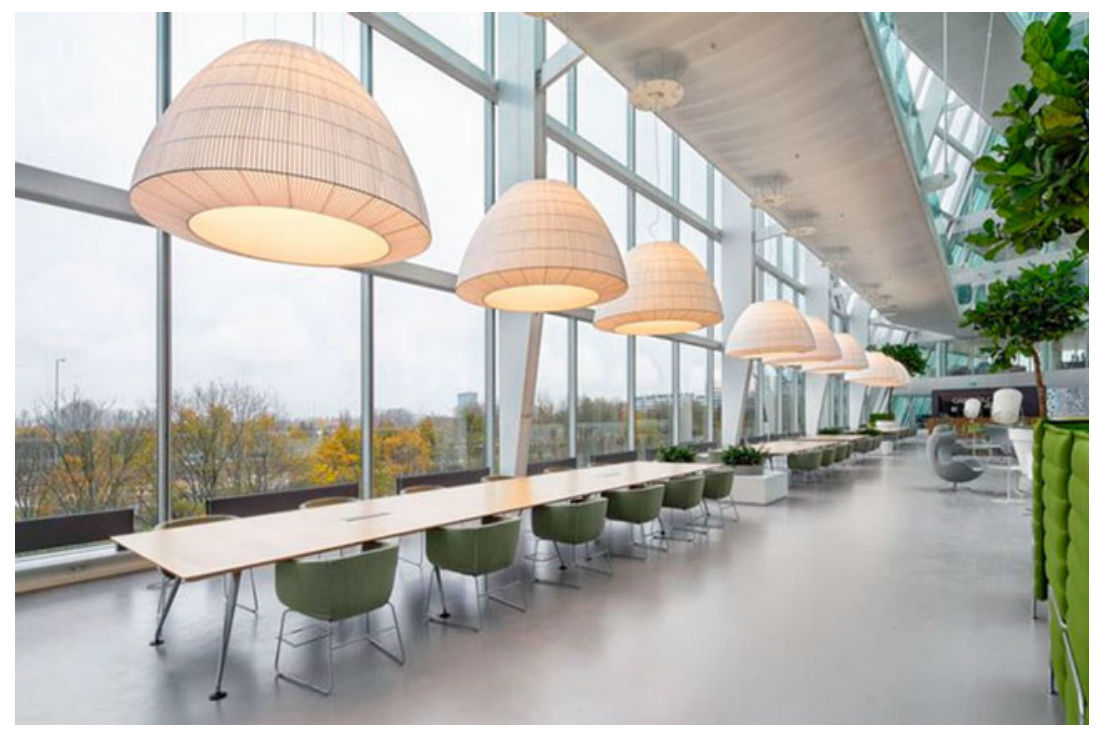

Abbildung 2.24 Deloitte Headquarter „The Edge“ (Quelle: OVG Real Estate)

gewonnen, um „The Edge“ zu heizen und zu kühlen. Auch für sämtliche Smartphones, Laptops und Elektroautos der Mitarbeiter*innen steht so mehr als genug Strom zur Verfügung. Regenwasser wird in „The Edge“ für die Bewässerung der Pflanzen und die Spülung der Toiletten gesammelt.

Passend dazu garantieren die durchgängige Glasfassade und große Flure, die das nördlich ausgerichtete, 15-stöckige Atrium umrahmen, lichtdurchflutete Arbeitsbereiche. Die Arbeitsbereiche sind dabei hell und großzügig gestaltet, wobei die Angestellten frei entscheiden können, ob sie im Großraum, am Stehpult, in der gemütlichen Sitzecke oder in einem Besprechungsraum arbeiten wollen. Mit dieser Vielzahl an unterschiedlichen Lern- und Arbeitsmöglichkeiten entspricht das Raumdesign den in Abschnitt 2.3.2 genannten Gestaltungsprinzipien.

Per Smartphone-App, die mit der Gebäudetechnologie und ihren etwa 20.000 Sensoren vernetzt ist, können Mitarbeiter*innen zum Beispiel die gewünschte Raumhelligkeit oder Temperatur einstellen. Darüber hinaus zeichnet die App sämtliche Aktionen, Vorlieben und Gewohnheiten der Angestellten auf und stellt sich danach stets auf deren individuellen Bedürfnisse ein. 
Ein bedeutendes Kriterium bei derart vernetzten und intelligenten Lernräumen ist eine „unaufdringliche Technik“. Sesink (2007) formuliert den Einsatz von Technik in Lernräumen folgendermaßen (Sesink, 2007, S. 3):

„Der Raum kann sagen: Hier geht es vor allem darum, Menschen an die Möglichkei-
ten der Technik heranzuführen; das entscheidende Entwicklungspotenzial für unsere
Zukunft liegt also in der Technik - wenn die Technik unübersehbar den Raum domi-
niert, vielleicht gar auf Podeste gestellt wird und die Bewegungsmöglichkeiten der
Menschen sich danach richten, wie man sich an die Technik begibt und an ihr aufhält
[...]. Der Raum kann aber auch sagen: Es geht um Euch, die Lernenden; es geht um
Menschen mit leiblichen Bedürfnissen; es geht um Zusammen-, Für- und Miteinander-
Lernen - wenn die Technik sich zurückhält, wenn die Raumgestaltung sich flexibel
unterschiedlichen Bedürfnissen der Nutzer anpassen lässt, wenn Menschen dort ihre
Spuren hinterlassen dürfen und sollen, wenn die Sinne der Nutzer angesprochen und
sie also in ihrer ganzen Existenz ernst genommen werden.“

An dieser Stelle entfaltet sich das Potential von IoT-Technologien, da diese i. d. R. durch ihre Miniaturisierung unauffällig in die vorhandene Architektur und das Mobiliar integriert werden können (Abbildung 2.25).

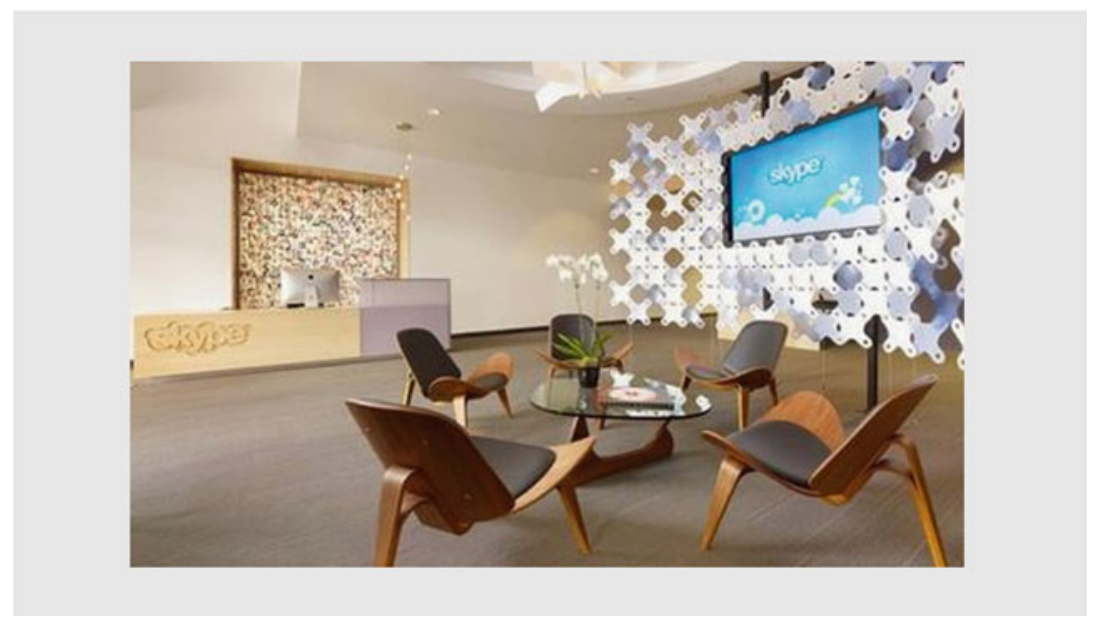

Abbildung 2.25 Skype Offices (Quelle: Skype) 
So ist es heutzutage möglich, Musik via Bluetooth Soundsystem über das Sofa ${ }^{17}$ abzuspielen oder Smartphone Ladestationen in die Tische zu integrieren (vgl. Ikea Qi-Technologie ${ }^{18}$ ). Die sogenannten Ladepads lassen sich überall einsetzen - ob auf dem Fensterbrett im Wohnzimmer, in einer Schublade im Schlafzimmer oder auf der Arbeitsfläche in der Küche. Zudem erforschen Möbelhäuser ${ }^{19}$ weitere Einsatzszenarien auf Basis Künstlicher Intelligenz.

Eine Integration von Technik in Architektur ist heutzutage annähernd Standard. So verfügen moderne Konferenzraumsysteme über Lautsprecher, die in die Wände oder Tische integriert sind. Eine derzeit noch eher visionäre Anwendung bezieht sich auf eine Multifunktionalität von Möbeln. So wurde beispielsweise bereits in 2009 im Rahmen eines Ideenwettbewerbs ${ }^{20}$ der Deutschen Initiative für Netzwerkinformation e. V. (DINI) zum Thema „Lebendige Lernorte“ ein multifunktionaler Tisch entworfen, der in einer vollvernetzten Studienumgebung eingesetzt werden soll. Der Tisch war Teil des Projektes „Learn connected! acht Visionen in verschiedenen Dimensionen“ und wurde von Studierenden der TU Dresden am Zentrum für Technisches Design entwickelt. Der multifunktionale Tisch (vgl. Abbildung 2.26) ist dabei Arbeits- und Präsentationswerkzeug in einem und verfügt über einen integrierten Bildschirm. Der digitale Lerntisch ist dabei in der Waagerechten als Schreibtisch mit integriertem Bildschirm und in der Senkrechten als Präsentationswerkzeug nutzbar.

Multifunktionale Raumkomponenten sind vielseitig nutz- und wandelbar und erfüllen didaktische Anforderungen in Bezug zur Methodenvielfalt. Beschreibund rollbare Trennwände dienen nicht nur der Strukturierung und Abtrennung im Raum sondern können gleichzeitig zum Anfertigen von Skizzen und Notizen dienen.

Sogenanntes „Smart Furniture 21 “ umfasst eine ganze Palette an wanderbaren Multifunktionsmöbeln, wo aus Regalen Tische werden oder sich Tische zu Sitzmöbeln umwandeln lassen. In Zukunft werden derartige Möbel in hohem Maße über digitale Zusatzfunktionen verfügen, so dass ein herkömmlicher Tisch, eine herkömmliche Wand oder auch Glas als Projektionsflächen und Touchscreens genutzt werden können.

\footnotetext{
${ }^{17} \mathrm{https}: / /$ bit.ly/2fYFq1A

${ }^{18}$ https://www.ikea.com/de/de/catalog/categories/departments/wireless_charging/

${ }^{19} \mathrm{https}$ ://www.abendblatt.de/wirtschaft/article210468063/Ikea-erwaegt-Einsatz-kuenstlic her-Intelligenz-in-seinen-Moebeln.html

${ }^{20} \mathrm{https}$ //dini.de/wettbewerbe/lebendige-lernorte/preisverleihung/?optout=1\&no_cache=1

${ }^{21} \mathrm{https} / / /$ creapills.com/designers-creatifs-maison-meubles-20171002
} 


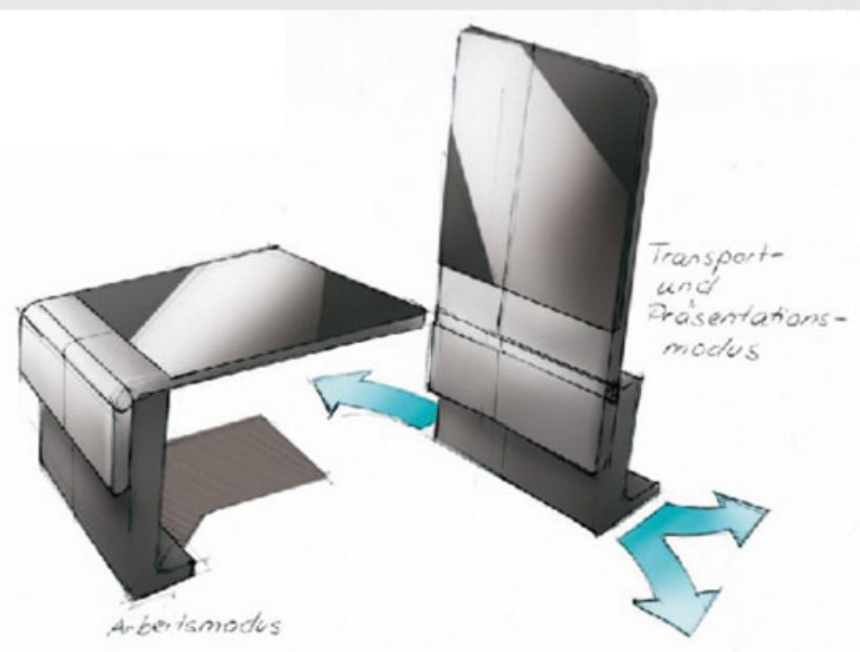

Abbildung 2.26 Entwurf zu einem multifunktionalen Lerntisch (Quelle: DINI e. V.)

Der SAP Data Space ${ }^{22}$ in Berlin ist ein IoT Accelerator für IoT-Startups und verfügt über einen Multi-Touch-Table (vgl. Abbildung 2.27) mit entsprechender Multi-Touch-Wall. Der SAP Data Space ist öffentlich zugänglich, sodass die neue Technologie erprobt und genutzt werden kann.

Zusammenfassend kann festgestellt werden, dass das Internet der Dinge mehr und mehr in den (Büro-)Alltag eindringt. Erste Studien belegen zudem positive Effekte auf Lehr- und Lernprozesse. Gomez et al. (2013) haben beispielsweise mit QR-Codes gearbeitet und physische Lerngegenstände mit entsprechenden digitalen Zusatzinformationen angereichert, die die Lernenden per QR-Code-Scanner abrufen konnten. Auf diese relativ einfach zu realisierende Weise konnten die Autoren die physische und virtuelle Welt verbinden und analoge Lerninhalte mit Multimedia anreichern (Gómez et al., 2013). Eine weitere Studie hat ergeben, dass eine automatisierte Steuerung von äußeren Faktoren wie Licht und Heizung bzw. Kühlung positiv auf Lernprozesse wirkt (Uzelac, Gligoric \& Krco, 2015). Bedenkt

\footnotetext{
${ }^{22} \mathrm{https}: / /$ dataspace-berlin.com/
} 
man jedoch, was IoT alles beinhalten könnte, kommt man zu dem Ergebnis, dass die empirische Forschung diesbezüglich noch am Anfang steht.

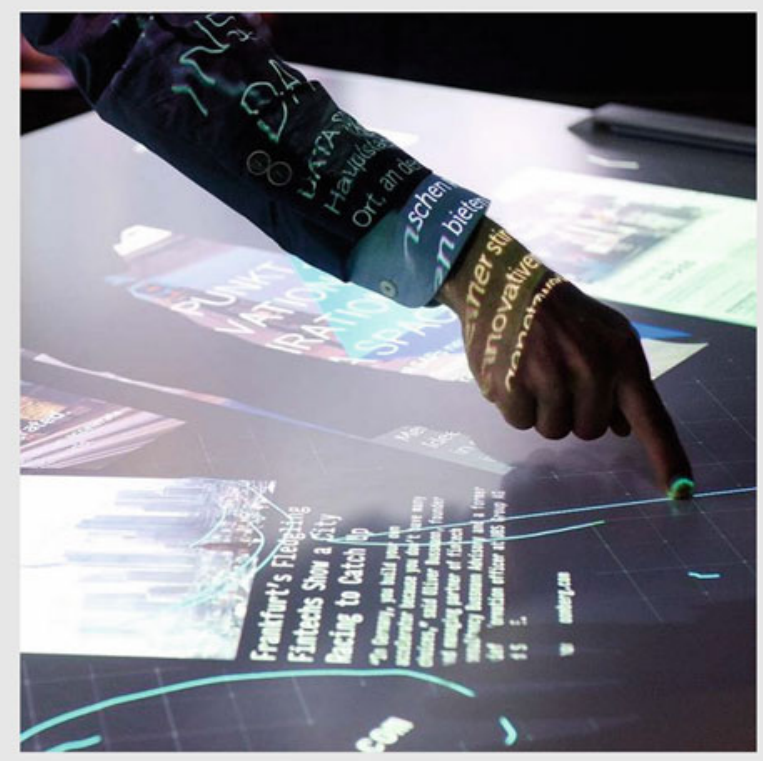

Abbildung 2.27 SAP Data Room mit e-Table (Quelle: SAP)

Neuere Produkte wie Amazon Echo ${ }^{23}$ bieten interessante Potenziale und sind die ersten ,,intelligenten Assistenten“ im Consumer Bereich, die sich schätzungsweise in den kommenden 5-10 Jahren rasant vermehren und v. a. auch technisch verbessern werden. Verbesserungen sind jedoch nicht nur im technischen Bereich, sondern auch in Datenschutzaspekten wünschenswert (vgl. Abschnitt 2.2.1.6).

Digitale Assistenten spielen bei intelligenten und hybriden Lernräumen eine wichtige Rolle. Sie werden schon seit vielen Jahren prototypisch entwickelt und scheitern derzeit noch an den hohen Entwicklungskosten. Rasante technologische Fortschritte könnten aber eine völlig neue Mensch-Maschine Interaktion auch in

${ }^{23}$ https://amzn.to/2hZ0QzJ 
Lernräumen ermöglichen. Im Jahr 2006 wurde z. B. der Nabaztag entwickelt. Nabaztag ist ein kommunizierender Gegenstand in Form eines stilisierten Hasen, der ähnlich wie Amazon Echo in der Lage ist, per Sprache FAktionen auszulösen (Domingo \& Forner, 2010).

In einem intelligenten und hybriden Lernraum könnte ein Nabaztag beispielsweise die Dozierenden unterstützen, indem er Präsentationen startet, Musik, Licht und Heizung steuert oder auch Dokumentationen mittels Videoaufzeichnung oder Photographien anfertigt (Abbildung 2.28).

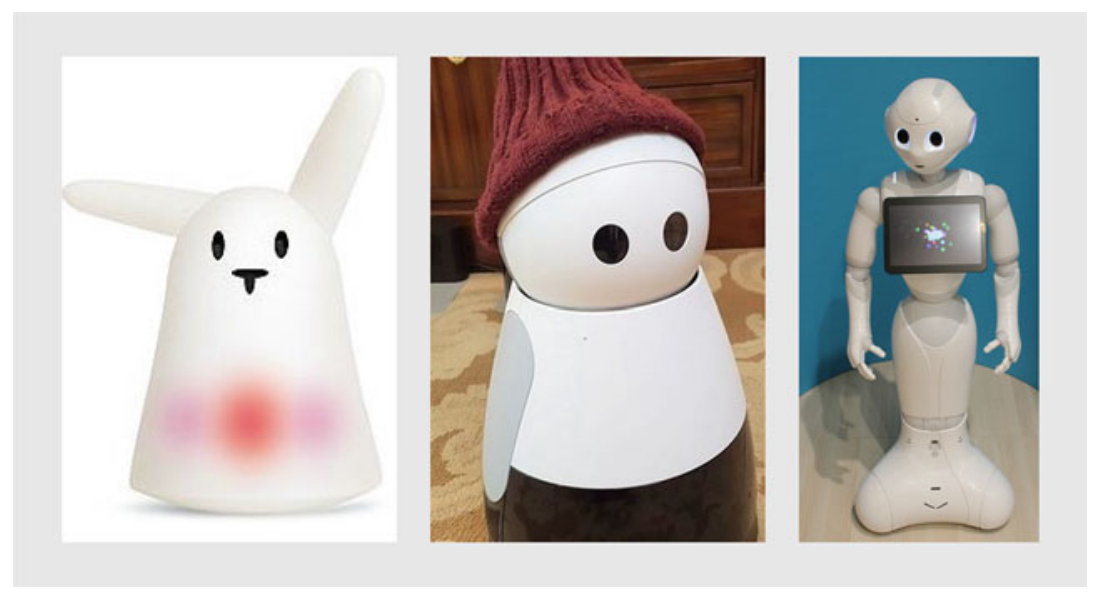

Abbildung 2.28 v.1.n.r. Roboter „Nabaztag“, „Kuri“ und „Pepper“

Roboter haben sich in den letzten Jahren vor allem im Haushaltsbereich rasant entwickelt, so dass frei navigierende Staubsaugroboter bereits in Media Märkten angeboten werden. Etwas hochwertigere Modelle wie z. B. Kuri von Bosch beinhalten darüber hinaus mehrere Funktionalitäten und verfügen über eine emotional ansprechende Optik, die eine Interaktion mit Menschen befördert.

Ein komplexerer humanoider Roboter ist beispielsweise der von dem französischen Unternehmen Aldebaran Robotics SAS und dem japanischen Telekommunikations- und Medienkonzern SoftBank entwickelte Pepper, der darauf programmiert ist, Menschen und deren Mimik und Gestik zu analysieren und auf diese Emotionszustände entsprechend zu reagieren. Er fungiert somit bereits als ein ,persönlicher Roboter“ (personal assistent) und wird am Campus der TH Wildau eingesetzt. Pepper ist in der Lage, kleine Bibliotheksführungen an der 
TH Wildau durchzuführen. Zudem kann er Fragen von den Gästen beantworten. Weitere Ausbaustufen sehen vor, dass zukünftig den Fahrstuhl nutzen oder den großen Touchscreen bedienen kann. Pepper ist programmierbar und durch die Hardwareausstattung vielfältig einsetzbar.

Für eine reibungslose und unsichtbar in den Alltag integrierte Umgebungsintelligenz (vgl. Abschnitt 2.2.1) wird es jedoch noch einige Jahre an Entwicklungsarbeit benötigen. Insbesondere wenn es darum geht, die derzeit vielfach proprietären Geräte untereinander und mit dem Internet bzw. den Robotern zu vernetzen.

Dennoch bietet das Internet der Dinge bereits heute völlig neue Möglichkeiten. Didaktisch sinnvolle Anwendungsbeispiele müssen jedoch erst identifiziert werden. So könnte es beispielsweise eine Smartphone-App geben, die anzeigt, welche Kollegen heute im Büro sind oder welche Besprechungsräume mit Videokonferenzsystem um 13:00 Uhr frei sind. Nach Besprechungsbeginn würde sie automatisch die externen Besprechungsteilnehmer anwählen, die Beleuchtung regeln und Signale geben, wenn die Zeit knapp wird. Sie könnte aber auch rechtzeitig auf das Ende der Besprechung hinweisen, damit noch genügend Zeit bleibt, alles aufzuräumen bevor das nächste Team ungeduldig vor der Tür steht. Doch wie wäre es erst, wenn der Raum in der Lage wäre, seine Nutzer*innen wiederzuerkennen, die Notizen der letzten Besprechung zu präsentieren und die bevorzugten Belichtungsszenarien einzustellen? Oder wenn er das aktuelle Besprechungsthema erkennt und auf Fachexperten im und außerhalb des Unternehmens hinweisen könnte? Oder bei Verspätungen eine kurze Nachricht an die wartenden Kollegen der nächsten Besprechung verschicken würde?

Das Institut für Multimediale und Interaktive Systeme (IMIS) der Universität zu Lübeck erforscht „Ambient Learning Spaces“. Dabei werden Lernprozesse durch vernetzte, interaktive, körper- und raumbezogene Medien unterstützt. Ambiente Lernumgebungen sind eine Form gemischter Realität (Mixed Reality), in der der Körper des Lernenden und der ihn umgebende Raum durch vernetzte digitale und personalisierte Medien angereichert werden (Winkler, Scharf, \& Herczeg, 2014). Die Forschergruppe fokussiert sich dabei nicht auf die Bereitstellung von Lerninhalten, sondern es geht vielmehr um eine Unterstützung selbstbestimmter, ganzheitlicher, eine Vielzahl der Sinne einbeziehenden, aktiven Aneignung von Kompetenzen seitens der Lernenden. Für die am Körper getragenen Medien werden Wearables (auch SmartFashion genannt) eingesetzt. Dabei werden informationstechnische Komponenten (z. B. LED's, Mikrokontroller, Sensoren, Batterien) in Kleidung, Schmuck oder Accessoires eingearbeitet.

Die Autoren Winkler, Scharf \& Herczeg (2014, S. 4) erläutern den Einsatz folgendermaßen: 
„So können beispielsweise in Bekleidung eingenähte Mikrokontroller derart programmiert werden, dass diese via Body Area Network (BAN) nicht nur mit anderen Wearables, vielmehr auch mit ALSApplikationen anderer Schalen, z. B. der peripheren Medien zugeordneten Applikation ActeMotion, via NEMO kommunizieren. Durch Berühren von Teilen der Bekleidung oder spezifische Bewegungen des Körpers können beispielsweise personalisierte oder narrativ zusammenhängende Medienobjekte aus NEMO während einer Performance auf einer Bühne projiziert werden.“

Während sich derartige IoT-Anwendungen aus dem Bereich FashionTech noch eher im Forschungsstadium befinden und sich wahrscheinlich gut im industriellen oder handwerklichen Bereich eignen, sind erste IoT-Lösungen in Büroumgebungen verfügbar.

So ist beispielsweise Ology ${ }^{\mathrm{TM}}$ eine Erinnerungsfunktion von Steelcase, die den Nutzer*innen Hinweise gibt, wann es an der Zeit ist, die Tischhöhe und die Körperhaltung zu verändern, damit sie nicht zu lange in einer ungesunden Position verharren. Ein entsprechendes LED-Display zur Steuerung ist flächenbündig in die Arbeitsoberfläche integriert. Die Intervalle der Hinweise sind abhängig von der Tischbelegung und von persönlichen Einstellungen, sodass alle Nutzer*innen ihren individuellen Rhythmus finden (Steelcase, 2016a).

Ein weiteres Beispiel ist die Brody® WorkLounge. Brody® WorkLounge ist ein separierter Mini-Arbeitsplatz, der mit speziellen Techniklösungen ausgestattet werden kann, die ein hochkonzentriertes Lernen fördern und Ablenkungen wirkungsvoll ausblenden. So wird konzentriertes Lernen und Arbeiten noch komfortabler, z. B. mit beheizbaren Sitzflächen, eingebauter Kopfstütze mit Lautsprechern etc. Integrierte Sensoren registrieren, wenn Brody besetzt ist und zeigen durch ein rotes Licht an, dass hier gerade gearbeitet wird. In Verbindung mit weiteren Applikationen (z. B. Workplace Advisor) liefert Brody Nutzungsdaten die anzeigen, wann und wie dieser Arbeitsplatz genutzt wurde.

Dies sind nur wenige konkrete Beispiele von etlichen weltweit (EDUCAUSE Learning Initiative (ELI), 2015). Um die Potenziale von IoT in Lernräumen systematisch $\mathrm{zu}$ erschließen gilt es zunächst, die Bedürfnisse der Lehrenden und Lernenden umfassend zu verstehen (Reinmann, 2012), um darauf aufbauend didaktisch sinnvolle Use-Cases prototypisch zu gestalten und zu testen, die dann in einem finalen Schritt in die Entwicklung überführt werden können. Die Bedeutung des User-Centered Designs stellen auch die Autoren Domingo und Forner (2010) ins Zentrum ihres Beitrages, der sich im Zusammenhang von IoT und eLearning mit einer Entwicklung hybrider Lernumgebungen beschäftigt. Darüber hinaus verweisen sie auf die Nützlichkeit von interdisziplinären Teams, um derart komplexe Lernarrangements zu entwickeln (Domingo \& Forner, 2010). 
In einer aktuellen Studie (Kanagarajan \& Ramakrishnan, 2017) werden alle weltweit bekannten ubiquitären und umgebungsintelligenten Lerninfrastrukturen identifiziert und miteinander vergleichen. Im Ergebnis stellen Kanagarajan \& Ramakrishnan fest, dass die identifizierten Beispiele, die sie differenzieren in Architektur, Ansatz, Analyse, Modell, Applikation und Gerät, nicht so intelligent sind, wie sie sein sollten. Sie sprechen in diesem Zusammenhang von einem „Smartness-Level“, der von ihnen definierte Kriterien erfüllen muss (vgl. Abbildung 2.29).

\begin{tabular}{|c|c|c|c|c|c|c|c|c|}
\hline Rocarchen & Self-alaptation & Sening & Samabc Learning & Inferring & Articipation & Selfeonfiguntion & Enagy Effickncy & Corted Awwenews \\
\hline Jones and to 2004 & $x$ & $\checkmark$ & $\gamma$ & $x$ & $x$ & $x$ & $x$ & $x$ \\
\hline Sdkanura and Koshinda 2005 & $\alpha$ & $\gamma$ & $\gamma$ & $\checkmark$ & $\gamma$ & $\alpha$ & $x$ & $\gamma$ \\
\hline Yang 2006 & $\checkmark$ & $\checkmark$ & $\alpha$ & $\checkmark$ & $\checkmark$ & $d$ & $x$ & 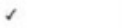 \\
\hline Yu et at 2008 & 1 & $\checkmark$ & 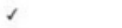 & $\checkmark$ & $\checkmark$ & $\alpha$ & $x$ & $\checkmark$ \\
\hline Znan and Yean 2009 & 1 & $\checkmark$ & 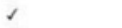 & $\checkmark$ & $\alpha$ & 1 & $x$ & $\checkmark$ \\
\hline Lecnidis et al. 2010 & $x$ & $\checkmark$ & 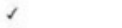 & $x$ & $x$ & $x$ & $x$ & $x$ \\
\hline Antona \& al. 2010 & $x$ & $\gamma$ & 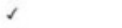 & $x$ & $x$ & $x$ & $x$ & $x$ \\
\hline Ramadan et al. 2010 & $x$ & $\checkmark$ & $d$ & $x$ & $x$ & $x$ & $x$ & $x$ \\
\hline Jin Ding a d. 2010 & $x$ & 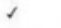 & $d$ & $x$ & $x$ & $x$ & $x$ & $x$ \\
\hline Gidhn and Spects 2010 & $x$ & 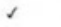 & 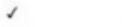 & $x$ & $x$ & $x$ & $x$ & $x$ \\
\hline Mandula et at 2011 & $x$ & 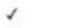 & $d$ & $x$ & $x$ & $x$ & $x$ & $x$ \\
\hline Wang and Wane 2011 & $x$ & $\checkmark$ & 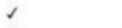 & $x$ & $x$ & $x$ & $x$ & $x$ \\
\hline Shith of al. 2012 & $x$ & $\checkmark$ & $d$ & $x$ & $x$ & $x$ & $x$ & $x$ \\
\hline Hwang et al 2012 & $x$ & $\gamma$ & 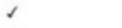 & $x$ & $x$ & $x$ & $x$ & $x$ \\
\hline Weal a al. 2012 & $x$ & $\checkmark$ & $\checkmark$ & $x$ & $x$ & $x$ & $x$ & $x$ \\
\hline Kowozi o al. 2012 & $\checkmark$ & $\alpha$ & $\checkmark$ & 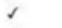 & $d$ & $\alpha$ & $x$ & 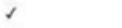 \\
\hline Martinet-Maldenado et al. 2013 & $\checkmark$ & 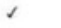 & 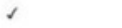 & $\gamma$ & $\checkmark$ & $\checkmark$ & $x$ & $\checkmark$ \\
\hline Mathioudakis a al. 2013 & $\checkmark$ & $\checkmark$ & $\checkmark$ & $\checkmark$ & $\checkmark$ & $\checkmark$ & $x$ & $\checkmark$ \\
\hline Atif et al. 2015 & $\checkmark$ & $\checkmark$ & $\checkmark$ & $\checkmark$ & $\checkmark$ & 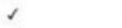 & $x$ & $\alpha$ \\
\hline Thipnk and Kuntach 2015 & $d$ & 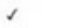 & $d$ & $d$ & $\checkmark$ & $\checkmark$ & $x$ & $\alpha$ \\
\hline Kadaivari and Sivahumar 2015 & 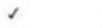 & $\checkmark$ & 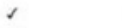 & $\checkmark$ & $\checkmark$ & 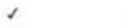 & $x$ & $\gamma$ \\
\hline Sujith and Sivakumar 2015 & 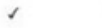 & $\checkmark$ & $\gamma$ & $\checkmark$ & $\gamma$ & $\checkmark$ & $x$ & $\checkmark$ \\
\hline
\end{tabular}

Abbildung 2.29 Smartness-Levels (Quelle: Kanagarajan \& Ramakrishnan, 2017)

Die Autoren plädieren schlussfolgernd dafür, das IoT Konzept nach Vermesan \& Friess (vgl. Abschnitt 2.2.1.4) für ubiquitäre Lernformen zu nutzen und formulieren dies wie folgt (Kanagarajan \& Ramakrishnan, 2017, S. 25):

„The IoT concept (Vermesan and Friess 2014) connects physical objects with one another to enable interaction among them and thus relieves the process of connecting end-user devices. IoT systems use cloud computing, low power sensors \& data reception electronic devices and distributed data communication frameworks."

In Anlehnung an bereits erläuterte IoT-Komponenten bzw. Funktionalitäten empfehlen Kanagarajan \& Ramakrishnan (2017) zudem die Integration eines 
IoT-Tisches, der über eine gestenbasierte Steuerungsmöglichkeit (Kinect), HD Kameras und einen Raspberry Pi verfügen soll (vgl. ebenda, S. 25):

\begin{abstract}
„The IoT based Ubiquitous Class room should be furnished with Ambient Light Sensors, AirQuality Sensor, RFID Sensor, Projector, Teacher's Control System, Microphones, Audio Systems, Interactive White Board, Visualizer, Solar panel power system and special purpose software (Data Analytics, Rich Multimedia processing, intelligent recognition). In addition, an IoT Desk [...] is also recommended to design with components such as Raspberry Pi3 Board, Low power Wi-Fi module, Kinect gesture recognition Sensor, HD Cameras, Near Field Communication (NFC) and monitor.“
\end{abstract}

Der von den Autoren genannte IoT-Tisch fungiert als Schnittstelle zwischen dem intelligenten und hybriden Lernraum und den darin wirkenden Lehrenden bzw. Lernenden. Für zukünftige Entwicklungen wäre es denkbar, die gleichen technischen Komponenten und Funktionalitäten in einen durch den Lernraum navigierenden Roboter zu integrieren, der sozusagen als mobiler, digitaler Assistent die Lehrenden und Lernenden per Sprach- und Gestensteuerung unterstützen kann.

In ihrer Untersuchung weisen Kanagarajan \& Ramakrishnan (2017) darauf hin, dass in den von ihnen analysierten Beiträgen das Thema Datenschutz und Datensicherheit völlig unbeachtet bleibt, obwohl es etliche Herausforderungen in diesem Zusammenhang gibt (vgl. Abschnitt 2.2.1.6). Aufgrund der elementaren Bedeutung für den Untersuchungsgegenstand, wird im folgenden Unterkapitel darauf eingegangen, welche datenschutzrechtlichen Aspekte bei der Gestaltung von intelligenten und hybriden Lernräumen berücksichtigt werden müssen.

\title{
2.3.4 Herausforderungen für Datenschutz und Datensicherheit
}

Die Datenmenge wächst und potenziert sich stetig. Für Wissensarbeiter*innen bedeutet dies sprichwörtlich, die Nadel im Heuhaufen zu finden. Das Problem ist der Datenmangel trotz Datenflut. Dies ist eine von vielen Herausforderungen, mit denen die Gesellschaft im Zuge der Transformation zur Wissensgesellschaft umgehen muss, nämlich diese verteilten, unstrukturierten Daten zur Wissensgenerierung handhabbar zu machen, indem geeignete Rahmenbedingungen für ein effektives, informelles Lernen am Arbeitsplatz entwickelt werden. Intelligente und hybride Lernräume (Smart Learning Environments) bieten jene geforderten Rahmenbedingungen. 
Unter Anwendung von Verfahren aus der künstlichen Intelligenz kann die Entwicklung und kontinuierliche Anpassung einer persönlichen Lernumgebung unterstützt werden. Die Informationsflut (Big Data) wird hierbei kanalisiert und vorstrukturiert, um Informationsprozesse effizienter zu gestalten (vgl. Abschnitt 2.1.2.5).

Wie in den vorhergehenden Abschnitten hergeleitet können digitale (Lern-) Assistenten oder auch SLEs die Mediennutzung in Lernprozessen der betrieblichen Weiterbildung durch „Empfehlungssysteme“ unterstützen. Die Auswirkungen können sowohl positiver (z. B. schnelleres Auffinden wichtiger Informationen, Zeitersparnis bei Recherchetätigkeiten etc.) als auch negativer Natur sein.

Kritisch zu betrachten ist, dass bereits die umfassende Recherche von Informationen einen Teil des Lernprozesses darstellt. Dazu gehört auch die Auswahl und Bewertung von relevanten Informationen. Dies ist eine wesentliche Grundkompetenz im Lernprozess selbst. Allerdings kann man davon ausgehen, dass Erwachsene diese Informationskompetenz im Zuge ihrer Grundqualifizierung bereits erworben haben und daher im Rahmen der betrieblichen Weiterbildung zu vernachlässigen ist. Darüber hinaus ist kritisch zu reflektieren, dass „Digitale Assistenten“ auf Grundlage der Auswertung von Big Data mittels (fremdgesteuerten) Algorithmen diesen Prozess der Informationsselektion sowie der Priorisierung übernehmen, so dass unter Umständen wichtige Informationen verloren gehen, die für den Lernenden relevant gewesen wären oder gar Manipulationen denkbar werden.

Technologisch betrachtet ist es möglich, dass derartige Systeme mit ihren Algorithmen auf Grundlage von bestimmten Interessensgruppen (z. B. während eines Wahlkampfes), zuvor festgelegte Inhalte favorisieren und ausschließlich eingeschränkte Inhalte den Systemnutzern ,empfehlen“ bzw. anzeigen. Im Kontext der betrieblichen Weiterbildung könnte ein derartiges Szenario beispielsweise das Auffinden von unternehmenskritischen Dokumenten unterbinden. Das bedeutet, dass selbst bei der Nutzung der sehr verbreiteten Google-Suche zu bedenken ist, dass die Treffer der Informationen und die Priorisierung der Darstellung auf Grundlage von zuvor festgelegten und fremdbestimmten Algorithmen zustande gekommen sind.

Davis (2017) führt darüber hinaus den Begriff von „Google-bombing“ auf. Darunter versteht man die Manipulation des Google-Suchergebnisses für eine bestimmte Webseite durch vielfaches Setzen von Links mit einem bestimmten, manipulativen Ankertext. In diesem Zusammenhang ist auch die gängige „Suchmaschinen-Optimierung“ als ein Werkzeug zur Einflussnahme auf die dargestellten und verfügbaren Informationen durch fremde Interessensgruppen bzw. 
Unternehmen zu betrachten. Grundsätzlich muss auch die Qualität von Big-Data selbst kritisch hinterfragt werden. Denn nur wenn die Qualität der zur Verfügung stehenden Daten hoch ist, kann das Ergebnis entsprechend sein (Davis, 2017). Bei unzureichender Datenqualität können die Ergebnisse bzw. empfohlenen Inhalte unter Umständen sogar falsch oder sinnlos sein.

Empfehlungssysteme, digitale Assistenten und selbst die Google-Suche basieren auf der Entwicklung von komplexen IT-Systemen mit dazugehörenden Algorithmen. Diese Systeme werden von Organisationen oder Institutionen programmiert und verfolgen entsprechende Ziele, die nicht unbedingt mit den Zielen der Nutzer*innen korrespondieren. Bereits Mark Weiser hat 1991 auf die kritischen Aspekte hingewiesen, die er wie folgt formuliert:

"Even today the [ubiquitous computing] could be a source of real harm in the wrong hands. Not only corporate superiors or underlings but also overzealous government officials and even marketing firms could make unpleasant use of the same information that makes invisible computers so convenient."

(Weiser, 1991, S. 104)

Neumann verweist im Zusammenhang der Softwareentwicklung auf absichtliche Sicherheitslücken, sogenannte „Blackdoors“. Diese bezeichnen einen (oft vom Hersteller eingebauten) Teil einer Software, der es Fremden bzw. Hackern ermöglicht, unter Umgehung der normalen Zugriffssicherung, Zugang zum Computer, den Daten oder einer sonst geschützten Funktion eines Computerprogramms zu erlangen. Einfache Bugs und unzureichende Kryptographie öffnen Hacker*innen Tür und Tor. Insofern plädiert Neumann insbesondere für Open Source Software, die über den offenen Quellcode partizipativ entwickelt und verbessert werden kann und so für größtmögliche Transparenz und Schutz sorgt (Neumann, 2014). In einer aktuellen Bestandsaufnahme zu Einsatzmöglichkeiten von IoT im Rahmen ubiquitärer Lernumgebungen verweisen darüber hinaus die Autoren Kanagarajan \& Ramakrishnan (2017) auf das Potenzial von Open Source Software und empfehlen, Hadoop, Moodle LMS, Bigblue button, Classflow und ThinkSpeak für die Entwicklung in Betracht zu ziehen. Des Weiteren wäre zu prüfen, inwiefern sich auch Blockhain als IoT-Infrastruktur nutzen lassen könnte.

Parallel dazu setzen adaptive und ubiquitäre Lernarrangements die kontinuierliche Erhebung, Verarbeitung, Speicherung und ggf. Weitergabe von personenbezogenen Daten voraus. Ein zentrales Element von SLEs ist dabei die Erstellung eines individuellen Lern- bzw. Qualifikationsprofils, das kontinuierlich verfeinert und angepasst wird. Dabei werden Informationen zur Person (Qualifikationen, berufl. Werdegang etc.), zum Aufenthaltsort, zum Surfverhalten, zu 
Interessensgebieten, zu beruflichen Tätigkeiten, zu Lernzielen, zu Netzwerken, zu Spezialkenntnissen etc. analysiert und ausgewertet (Bomsdorf, 2005; Brusilovsky \& Peylo, 2003; Hwang, 2014; Katzlinger, 2007).

Insofern ist eine ausgeprägte Digital- bzw. Internetkompetenz sehr wichtig bei der Nutzung derartiger Systeme, um kritisch zu hinterfragen, wer z. B. Smart Learning-Produkte oder Services anbietet und auf welcher Datengrundlage die Services zur Verfügung gestellt werden. Umfassende Informations-, Digital- und Medienkompetenz sind sozusagen die Grundlage und wichtige Schlüsselqualifikationen für Lernende im digitalen Zeitalter.

Das Sammeln und Auswerten von (personenbezogenen) Daten hat jedoch nicht nur fundamentale Auswirkungen auf den Einzelnen, sondern auf die gesamte Gesellschaft (Schaar, 2014). Dies ist ein Kernproblem, welches nur über die Herstellung maximaler a) Transparenz, b) Datensouveränität und d) Selbstbestimmung der Nutzer*innen überwunden werden kann. Insbesondere bei der Gestaltung von Bildungsinnovationen wie SLEs bietet der „Privacy by Design-Ansatz“ konkrete Lösungswege, um o. a. Hindernissen zu begegnen (Langheinrich, 2001).

Welche gesellschaftspolitischen Veränderungen im Zusammenhang von Big Data im Bildungsbereich zu erwarten sind beschreiben die Autoren MayerSchönberger \& Cukier (2014) an einem konkreten Fallbeispiel des „Prof. Ng“, einem Hochschullehrenden, der alle Informationen seiner Studierenden sammelt. Mit Hilfe dieser Daten kann Prof. Ng dann Systeme entwickeln, welche diese Erkenntnisse dann automatisch an die Studierenden zurückspielen, womit der Unterricht sowie die Stoffbeherrschung und Leistungsfähigkeit allgemein verbessert wird. Darüber hinaus verfolgt Prof. $\mathrm{Ng}$, wie die Studierenden mit den Videolektionen umgehen, was sie sich mehrfach ansehen, wo sie zurückspulen etc. Er zeichnet die Hausaufgaben und Tests auf, die über Laptops und Tablets gemacht werden, und stellt so genau fest, bei welchem Thema individuelle Unterstützung benötigt wird. Zudem kann er die Daten seiner sämtlichen Kurse aus den Vorjahren gemeinsam auswerten, um zu ermitteln, was am besten funktioniert und bei welchen Teilen es Schwierigkeiten gibt.

Professor $\mathrm{Ng}$ konnte so herausfinden, dass Studierende in einer bestimmten Lektion Schwierigkeiten hatten und daher eine andere frühere Lektion erneut aufriefen. Nach dieser Erkenntnis baute er seinen Kurs um und wiederholte genau die häufig aufgerufene Stelle in der früheren Lektion erneut. Konnten die Studierenden eine bestimmte Aufgabe nicht lösen, analysierte Prof. Ng die Daten und stellte fest, dass einige Studenten jedoch nach dem Lesen eines bestimmten Foreneintrags die Aufgabe danach lösen konnten. Daraufhin zeigte das System, wenn 
es feststellte, dass ein Student Schwierigkeiten mit einer Aufgabe hatte, automatisiert genau eben jenen Foreneintrag zum Nachlesen an, der vielen anderen Studierenden vorher bereits geholfen hatte.

Professor $\mathrm{Ng}$ änderte damit sein Lehrkonzept insofern, dass er nicht mehr ausschließlich danach ging, Beiträge die von den Studierenden am besten bewertet wurden zu verwenden, sondern er wählte datengesteuert diejenigen Inhalte heraus, die das Lernen gemäß seinen Analysen am besten unterstützten (MayerSchönberger \& Cukier, 2014)

Die in diesem Kontext auftretenden datenschutzrechtlichen Fragen sind auch für IT-gestützte Weiterbildungssysteme im betrieblichen Umfeld relevant, die Learning Analytics anwenden.

In einem gewissen Spannungsfeld zum oben genannten Beispiel steht das Thema Datenschutz, welches dem Schutz der Persönlichkeit (insb. Sozial-, Privats- und Intimsphäre, Ruf, Ehre, Darstellung, etc.) und damit verbunden auch der Gewährleistung der allgemeinen Handlungsfreiheit der Menschen dient. Bereits im Jahr 1983 entwickelten die Richter des BVerfG in Karlsruhe das „Grundrecht auf informationelle Selbstbestimmung“, da sie in der aufkommenden automatisierten Datenverarbeitung die Gefahr sahen, dass die bloße Registrierung persönlicher Daten bei den Bürgern zu der Befürchtung führen könnte, dass ihre personenbezogenen Daten ohne ihr Wissen ausgewertet, weitergegeben und langfristig gespeichert werden. Dies wäre nach Ansicht der damaligen Verfassungsrichter ein die Funktionsfähigkeit einer freien Gesellschaft gefährdender Umstand, da in der Folge die Bürger ihr gesellschaftliches Verhalten entsprechend dieser Befürchtungen anpassen würden, d. h. sich demgemäß nicht mehr „frei bewegen“ und damit nicht mehr „frei entfalten“ würden ${ }^{24}$.

Die Grundprinzipien des Datenschutzes sind bis heute das „Verbot mit Erlaubnisvorbehalt“ (informierte Einwilligung oder gesetzliche Grundlage für eine rechtmäßige Datenerhebung, -verarbeitung und -nutzung erforderlich), die „Zweckbindung“, die „Datensparsamkeit“, die tatsächliche „Erforderlichkeit“ der personenbezogenen Daten für den konkreten beabsichtigten Verarbeitungszweck und die „Transparenz“ gegenüber den Betroffenen über die Verarbeitung der Daten.

Die detaillierte Beobachtung von menschlichem Verhalten und anschließende Analyse und Auswertung in Big Data Massendatenanwendungen und vor allem der Umstand, dass Daten nicht zu einem bestimmten Zweck erhoben, verarbeitet

\footnotetext{
${ }^{24}$ vgl. Art. 2 Abs. 1 iVm. Art. 1 Abs. 1 GG bzw. auch im Europäischen Primärrecht vgl. Art. 1 u. Art. 8 Charta der Grundrechte der Europäischen Union
} 
und genutzt werden, sondern man bei der Erhebung noch gar nicht weiß für welche weiteren Zwecke man die Daten verwenden möchte, sind schwer mit diesen fundamentalen datenschutzrechtlichen Grundsätzen der ,Zweckbindung“, „Datensparsamkeit" und „Transparenz" zu vereinbaren (Bitter, Buchmüller \& Uecker, 2014; Feiler \& Fina, 2013; Ohrtmann \& Schwiering, 2014).

Die Anwendung von Big Data Analysen birgt neben den enormen Vorteilen folglich die Gefahr, dass der Schutz der Privatsphäre nicht nur schwieriger wird, sondern weitere Risiken neu hinzukommen, insbesondere, dass Menschen aufgrund von Big Data Analysen diskriminiert und aufgrund von Vorhersagen vorab ungerechtfertigt bestraft werden.

Aus Datenschutzsicht besteht nun die Herausforderung darin, die berechtigten Interessen der verantwortlichen datenverarbeitenden Stelle an Big Data Analysen mit dem Recht auf informationelle Selbstbestimmung der Betroffenen ins Gleichgewicht zu bringen oder eine datenschutzrechtlich konforme Einwilligung einzuholen.

Dazu müssen die konkreten Zwecke der Datenanalysen von Beginn der Erhebung, Verarbeitung und Nutzung sehr ausführlich, transparent und so verständlich wie möglich dargestellt werden und die Ausübung von Betroffenenrechte (Recht auf Auskunft, Widerspruch, Berichtigung und Löschung) muss gewährleistet werden können. Eine personenbezogene Datenspeicherung auf Vorrat für spätere Big Data Auswertungen widerspricht aktuell dem Grundsatz der Zweckbindung und, dass personenbezogene Daten nicht länger, als es für die Realisierung der Zwecke, für die sie erhoben oder weiterverarbeitet werden, erforderlich ist, in einer Form aufbewahrt werden, die die Identifizierung der betroffenen Personen ermöglicht.

Die neue ab 25. Mai 2018 geltende Datenschutz-Grundverordnung (EU) 2016/679 (DSGVO) ermöglicht nun eine Weiterverarbeitung ohne neue Rechtsgrundlage, wenn eine ,Vereinbarkeit mit dem ursprünglichen Verarbeitungszweck $^{\text {“ gegeben ist }}{ }^{25}$. Dies wird es in Zukunft erleichtern können, Big Data Anwendungen $\mathrm{zu}$ betreiben, die datenschutzrechtlich gesehen mit dem ursprünglichen Verarbeitungszweck der Daten ,zu vereinbaren“ sind.

Wie die Autoren Mayer-Schönberger \& Cukier (2013) zutreffend ausführen, reicht heute das Datenschutzrecht alleine aber nicht mehr aus bzw. ist auch mit diesen neuen technischen Entwicklungen nicht mehr völlig in Einklang zu bringen, wie das Beispiel der Einwilligungen zeigt (Mayer-Schönberger \& Cukier, 2013):

${ }^{25}$ vgl. Art. 5 Abs. 1 lit. b iVm. Art. 6 Abs. 4 DSGVO 
- Wie kann der Einzelne eine Zustimmung zu etwas geben, was er noch gar nicht kennt?

- Wie können verantwortliche Stellen Betroffene von Datenverarbeitungen in Kenntnis setzen, wenn die Verwendung noch unklar ist?

Big Data Analysen und Vorhersagen bergen darüber hinaus die große Gefahr, dass Menschen weiterhin die Welt durch die Brille von Ursache und Wirkung sehen, obwohl Big Data auf Korrelationen beruht und damit für die Beurteilung von Kausalität und Schuld gänzlich ungeeignet ist. Big Data Anwendungen könnten demgemäß für kausale Zwecke missbraucht und mit gefährlichen Visionen von gesteigerter Effizienz durch auf Big-Data-Vorhersagen gestützten Urteilen in Zusammenhang gebracht werden. Die Denkweise im Zusammenhang mit Big Data Auswertungen und Vorhersagen muss sich in der Form verändern, dass man sich von den gewohnten Vorstellungen von Kausalität und Schuld entledigen wird müssen und sich stattdessen vermehrt auf Korrelationen verlassen wird müssen.

Man wird bei Big Data Analysen oft nicht mehr wissen „warum“ sondern nur mehr „was“. Der Umbruch der Denkrichtung von „warum“ zu „was“ verändert dabei tiefgreifend die Art, wie Menschen Entscheidungen treffen. Das Besondere an Big Data ist dabei die neue Qualität der Ergebnisse aus der Kombination bisher nicht aufeinander bezogener Daten ohne aber letztlich genau zu wissen ,warum“ etwas so ist, sondern nur „was“ ist (Horvath, 2013).

Big Data Auswertungen und damit verbundene Vorhersagen wirken sich dabei auch massiv auf digitales Lernen aus, denn die umfassend gesammelten und ausgewerteten Lerndaten (Learning Analytics) könnten dazu verwendet werden, Vorhersagen über unsere Zukunft zu treffen. Dies könnte einerseits die Lernfreiheit beschränken, im schlimmsten Fall dazu führen, dass ein Lernsystem zur Vorhersage kommt, man werde scheitern und Lernende unterschwellig zum Abbruch bewegt ohne aber zu wissen warum eigentlich, da Big Data wie eben hergeleitet rein auf Basis von Korrelationen Vorhersagen trifft (Mayer-Schönberger \& Cukier 2014).

Darüber hinaus ist es erforderlich, dass die Löschung bzw. Anonymisierung von Altdaten sichergestellt wird. Während Menschen die Fähigkeiten besitzen nach einer gewissen Zeit zu vergessen, bleibt die Gefahr, dass die Daten über unser Lernverhalten permanent verfügbar bleiben und wir im Sinne einer „Permanenz der Vergangenheit" keine Chance haben, Vergangenes entsprechend relativieren zu können (vgl. ebenda). Die neue Datenschutz-Grundverordnung (EU) 2016/679 versucht dieser Problematik mit dem Recht auf Löschung (,,Recht auf Vergessenwerden") in Art. 17 DSGVO zu lösen. Eine betroffene Person hat 
das Recht, die Löschung der sie betreffenden personenbezogenen Daten zu verlangen. Art. 16 DSGVO gibt zudem das Recht auf Berichtigung, das heißt, eine betroffene Person hat ein Recht, die Berichtigung sie betreffender unrichtiger personenbezogener Daten zu erwirken.

Zudem wird ein Recht auf Datenübertragbarkeit (Art. 20 DSGVO) neu eingeführt. Dies bedeutet, dass eine betroffene Person auch das Recht hat, die sie betreffenden personenbezogenen Daten, welche sie einem für die Verarbeitung Verantwortlichen zur Verarbeitung bereitgestellt hat, in einem strukturierten, maschinenlesbaren Format zu erhalten und diese Daten einem anderen Verantwortlichen ohne Behinderung zu übermitteln. Dies führt dazu, dass es damit in Zukunft datenschutzrechtlich möglich sein wird, Smart Learning Environments $\mathrm{zu}$ wechseln und Lernende dabei das Recht haben, die entsprechenden Daten mitzunehmen.

Jedoch sind von diesem Recht auf Datenübertragbarkeit nur jene personenbezogenen Daten betroffen, welche die betroffene Person willentlich und wissentlich an den für die Verarbeitung Verantwortlichen übermittelt hat. Insofern bleibt unklar, ob Analysedaten aus der Beobachtung des eignen Lernverhaltens durch einen datenschutzrechtlich Verantwortlichen auch unter dieses Recht auf Datenübertragbarkeit fallen werden, insbesondere, wenn sich Lernende wissentlich und willentlich zur Verbesserung der eigenen Lernleistung umfangreich beim Lernen vom Verarbeiter beobachten lassen. Es könnte nämlich auch argumentiert werden, dass eben nur solche Daten vom Recht auf Datenübertragbarkeit erfasst werden, wo Lernende selber etwas aktiv geschrieben und/oder gesprochen haben und damit diese Daten selber bereitgestellt haben, während Daten der Beobachtung des Lernverhaltens durch den für die Verarbeitung Verantwortlichen selbst erhoben werden und eben gerade nicht von den Lernenden selber ,,bereitgestellt“ werden.

Aus den eben skizzierten datenschutzrechtlichen Herausforderungen lässt sich zusammenfassend festhalten, dass Big Data und IoT-Technologien einerseits die Qualität von Lernumgebungen wie bspw. durch Smart Learning Environments deutlich steigern können, andererseits aber gravierende Herausforderungen eröffnen. Dieser Umstand manifestiert sich in einem Spannungsfeld, wobei zwischen einer pädagogisch wünschenswerten Grundkonzeption von SLEs und den gesetzlichen Rahmenbedingungen was den Datenschutz betrifft balanciert werden muss (Alonso \& Arranz, 2016).

Folglich ergibt sich in Bezug auf Datenautonomie und Datensicherheit die Notwendigkeit einer frühzeitigen und differenzierten Auseinandersetzung mit dem Thema. Hierbei sind (bekannte) Sicherheitslücken (Neumann 2014) sowie datenschutzrechtliche Aspekte gleichermaßen zu berücksichtigen. 
Zum Schutz der Privatsphäre und unter Gewährleistung des Grundrechts auf informationelle Selbstbestimmung sind Lösungsansätze erkennbar, die sich in den folgenden Prinzipen widerspiegeln und im Rahmen von „Privacy by Design“ parallel zur Datenschutz-Grundverordnung im Gestaltungsprozess von SLEs berücksichtigt werden sollten:

1. Aufklärung der Nutzer*innen

2. größtmögliche Anonymisierung

3. größtmögliche Löschung

4. klare Regelungen \& Rollen (Anpassung und Modernisierung des Datenschutzrechts)

5. größtmögliche Transparenz

6. größtmögliche Datenautonomie bei Nutzer*innen: Eröffnung von Handlungsspielräumen und Modifizierungsmöglichkeiten

7. Vermeidung monopolartiger Strukturen (Google, Facebook...)

8. Prüfung von Sicherheitslücken

9. Einsatz kryptographischer Verfahren

10. keine Urteile über Menschen aufgrund von Big Data Vorhersagen

11. Sicherstellung, dass Big Data Analysen nicht zur Beurteilung von Kausalität und Schuld herangezogen werden (im Sinne von ,warum“, sondern rein zum Aufdecken von Korrelationen im Sinne von ,was?“)

Damit Datenschutz und Datensicherheit nicht als Hindernis von Innovation empfunden werden, könnte ein Lösungsansatz in der konsequenten Förderung von Digitalkompetenzen sowie in der stringenten Verfolgung des „Privacy by Design“-Ansatzes liegen, der bereits vor der Entwicklung von Systemen datenschutzrechtliche Aspekte im Vorfeld analysiert, beschreibt und zum Wohle der Nutzer*innen durch Berücksichtigung o. a. Prinzipien gestaltet.

\subsection{Theoretische Synthese im Lernformat „Smart Learning Environments"}

Nachdem in vorhergehenden Abschnitten interdisziplinäre Grundlagen in den Bereichen Lehr- und Lernforschung, Internet der Dinge sowie Lernraumforschung gelegt wurden, anhand derer in Abschnitt 2.2.3 gezeigt werden konnte, dass durch die Übertragung von IoT auf Lehr- und Lernprozesse das Lernformat „Smart Learning Environments" resultiert, werden die dargestellten Grundlagen nunmehr 
in einer theoretischen Synthese zu Smart Learning Environments zusammengeführt. Dabei erfolgt zunächst die Darstellung zum aktuellen Stand der Smart Learning Environments-Forschung sowie anschließend ein Diskurs hinsichtlich vorhandener Gestaltungsverfahren im Kontext einer organisationalen Entwicklung und Nutzung von SLEs, die abschließend in der Darstellung der Forschungslücken münden. Da es sich bei SLEs um ein sehr junges Forschungsfeld handelt, das sich überwiegend auf die in 2014 initiierte International Association for Smart Learning Environments (IASLE) gründet, ist der Forschungsstand im Vergleich zu den Abschnitt 2.1, 2.2 und 2.3 überschaubar.

Die IASLE $^{26}$ ist eine internationale Vereinigung zum Forschungsfeld der Smart Learning Environments und bietet ein professionelles Forum für Forscher, Akademiker, Praktiker sowie Fachleute aus der Wirtschaft, die an einer innovativen Neugestaltung von Lehr- und Lernformen interessiert sind. Wissenschaftliche Publikationen zum Thema werden im Smart Learning Environments Journal ${ }^{27}$ gebündelt.

Ziel der IASLE ist der fachliche Austausch und die Förderung von Verfahren, die zur Entwicklung, Gestaltung und Umsetzung von intelligenten Lernumgebungen beitragen. Dem Zusammenspiel von interdisziplinären Fachdisziplinen wie z. B. Pädagogik und Informatik wird dabei eine große Bedeutung zugeschrieben.

\subsubsection{SLEs als Forschungsgegenstand}

Bei der Forschung zu SLEs handelt es sich um ein interdisziplinäres Feld. Neben der (Wirtschafts-)Informatik beschäftigen sich weitere sozialwissenschaftliche Disziplinen mit Smart Learning Environments als Forschungsgegenstand so z. B. die (Medien-) Pädagogik oder Psychologie. Entsprechend gibt es unterschiedliche Zugänge und Definitionen von SLEs, die nachfolgend dargelegt werden. Für die Literaturanalyse wurden Beiträge ausgewählt, die sich explizit mit der Konzeption und Entwicklung, Gelingensbedingungen sowie Anforderungen an Gestaltungsprozesse von SLEs auseinandersetzen. Nicht berücksichtigt wurden Beiträge, die sich im weitesten Sinne mit „Smart Learning“ beschäftigen, da diese theoretischen Grundlagen bereits in Kapitel 2 abgebildet wurden. Die systematische Literaturanalyse identifizierte bis September 2016 insgesamt 5 Schlüsselwerke, die im Folgenden beleuchtet und diskutiert werden.

\footnotetext{
${ }^{26}$ https://iasle.net/about-us/mission/

${ }^{27} \mathrm{https}$ ///slejournal.springeropen.com/
} 
Als Vorläufer der SLE-Forschung, die seit 2014 im Journal for Smart Learning Environments publiziert wird, ist der Beitrag von Lei, Wan \& Man (2013) aufzuführen. Dieser Aufsatz ist insofern relevant für die vorliegende Untersuchung, da die Autoren Smart Learning Environments auf Grundlage von Cyber-Physischen Systemen entwickeln und so auf eine in der Literatur selten direkt benannte Verbindung zu IoT hinweisen (vgl. Abschnitt 2.2.1.3). Lei, Wan \& Man (2013) beschreiben ein SLE, das mittels „,smarter Applikationen“ in einer Hochschule eingesetzt wurde. Dabei kam ein Cyber-Physisches System zum Einsatz, das über Embedded Systems (vgl. Abschnitt 2.2.1.2) unterschiedliche Arten von Sensoren und Aktoren vernetzte und spezifische Funktionen automatisiert steuerte. Dabei wurden äußere, lernförderliche Rahmenbedingungen wie Beleuchtung, Heizung und Luftqualität kontrolliert und automatisiert angepasst sowie die Energieeffizienz deutlich gesteigert (Lei et al., 2013). Der Beitrag zeigt, wie auf eine relativ einfache Art und Weise „smarte Funktionalitäten“ in Lernräume integriert und lernförderliche Rahmenbedingungen unterstützt werden können, die wie in Abschnitt 2.3.2 dargelegt, das Lernen positiv oder auch negativ beeinflussen können.

Im Rahmen eines "preliminary framework for smart learning environments" (vgl. Abbildung 2.30) führt Spector (2014) philosophische, psychologische und technologische Ansätze zusammen, um eine Grundlage zur Planung und Implementierung von SLEs zu entwickeln. Intelligente Lernumgebungen müssen nach Ansicht des Autors bedeutsam sein und den von ihm postulierten ,3Es“ gerecht werden. Spector (2014) zufolge müssen Smart Learning Environments eine nachhaltige Bildungsarbeit auf folgenden Ebenen erfüllen:

1. Engagement

2. Effectiveness

3. Efficiency

Dabei spannt er einen Bogen ausgehend von erkenntnistheoretischen Paradigmen, über pädagogische Anforderungen bis hin zu wirtschaftlichen Interessen (Spector, 2014).

Er plädiert für eine stärker strategisch ausgerichtete Planung und Gestaltung von SLEs. In diesem Zusammenhang führt er auf, dass man sich zunächst damit beschäftigen muss, wie menschliche Lernprozesse funktionieren und wie Menschen letztlich Wissen und Kompetenzen aufbauen können. Diesbezüglich verweist er u. a. auf Erkenntnisse aus der pädagogischen Psychologie, die systematisch in den Gestaltungsprozess zu integrieren sind. Technologie muss nach 


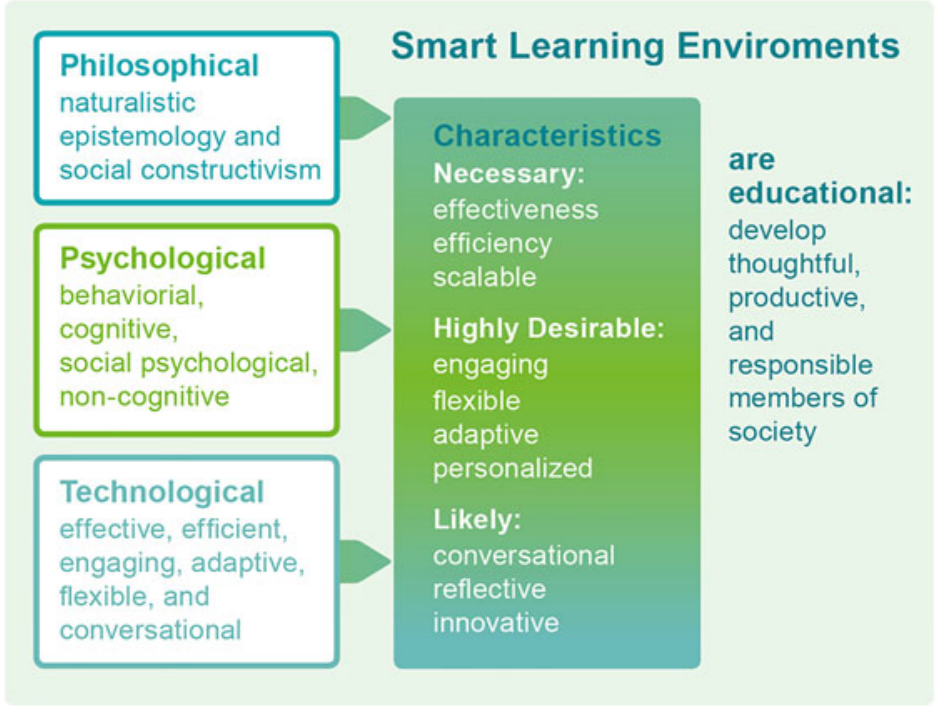

Abbildung 2.30 Preliminary Framework for SLEs (Quelle: Nach Spector 2014)

Spector (2014) die oben aufgeführten 3Es unterstützen, also Engagement, Effectiveness und Efficiency. Somit erhielte die Technologie einen Sinn und einen spürbaren Nutzen.

Die Bedeutung von SLEs stellt Spector (2014) letztlich in den Kontext einer gesellschaftlichen Verantwortung in dem Sinne, dass SLEs dazu beitragen müssten, die Menschen zu nachdenklichen, produktiven und rücksichtsvollen Mitgliedern der Gesellschaft zu entwickeln.

Im Vergleich zu dem von Spector generisch beschriebenen Meta-Konzept, das einem deduktiven Vorgehen gleicht und bei welchem eine Perspektive von außen eingenommen wird, entwickelt Koper (2014) ein neues theoretisches Konzept zur Gestaltung von SLEs, das von den konkreten Interaktionen zwischen Mensch und Technik ausgeht. Das auf einem eher induktiven Vorgehen beruhende Konzept, das an Konzeptionen von User Interfaces (Human Computer Interaction) anknüpft, bezeichnet Koper entsprechend als „Human Learning Interfaces (HLIs)“ (Koper, 2014).

Um von User Interfaces auf Human Learning Interfaces innerhalb von SLEs zu schließen, differenziert Koper (2014) zunächst unterschiedliche Lernumgebungen im Grad der digitalen und analogen Lern-Stimulation: 
1. The zero case: wenn die Lernumgebung keine digitalen oder physischen LernStimuli bietet.

2. The digital case: wenn die physische Umgebung digitale Lerngeräte enthält, den Lernenden jedoch keine relevanten nicht-digitalen Stimuli liefert.

3. The embedded case: wenn die physische Umgebung den Lernenden relevante Stimuli liefert und gleichzeitig die digitalen Geräte situationsbezogene Zusatzinformationen ergänzen, um die kognitive Verarbeitung und Repräsentation der Inhalte im Gehirn zu verstärken. In diesem Fall gibt es eine kombinierte, teilweise digital, teilweise physisch stimulierte Lernumgebung. In der vorliegenden Arbeit wird dies im Folgenden als Hybridisierung bzw. hybridisiertes Lernen bezeichnet.

4. The side-by-side case: wenn in einer physischen Umgebung digitale Lerngeräte genutzt werden, um zusätzliche Lernfunktionen wie Recherche, Interaktion oder Tests zu ermöglichen, die digitalen Geräte aber die tatsächliche physische Umgebung bzw. Situation nicht erkennen können. In diesem Fall ist die Lernumgebung fragmentiert und in voneinander getrennte physische und digitale Einheiten aufgeteilt.

5. The classical case: wenn die physische Umgebung relevante Reize liefert, es aber keine zusätzlichen digitalen Stimuli gibt.

Schlussfolgernd klassifiziert Koper (2014) SLEs als „Embedded Case“ (vgl. 2.2.1.2) und skizziert für diese spezifische Lernumgebung protypische Anforderungen, auf Basis derer SLEs entwickelt werden können:

1. Digitale Geräte werden den physischen Lernorten hinzugefügt

2. Die digitalen Geräte erkennen den Standort und den Kontext der Lernenden

3. Die digitalen Geräte erweitern die physische Lernumgebung mit zusätzlichen, digitalen Lernfunktionen

4. Die digitalen Geräte überwachen den Fortschritt der Lernenden

Ziel von Smart Learning Environments ist es nach Koper, ein schnelleres und besseres Lernen zu ermöglichen (2014).

Entsprechend analysiert er die Bedingungen, die dies ermöglichen und entwickelt auf Basis des „modal model of the architecture of human information processing (Ashcraft \& Radvansky, 2010, S. 38) ein „Human Learning Interfaces“ (HLIs) Konzept (vgl. Abbildung 2.31). Auf Grundlage der „Information Processing Theory“ (vgl. Abschnitt 2.1.1) identifiziert er zunächst in Anlehnung an Human Computer Interaction vier Hauptinteraktionen, die zwischen einem Digitalen Gerät und dem Lernenden stattfinden können: 
Abbildung 2.31 Five Types of Human Learning Interfaces (Quelle: Nach Koper 2014)

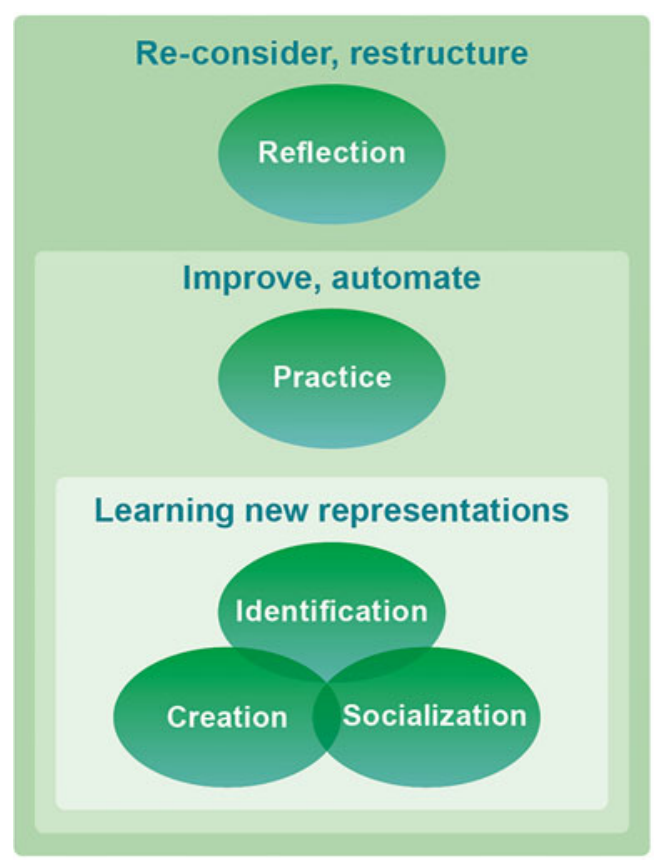

1. Fragen stellen

2. Aufgaben geben

3. Informationen zur Verfügung stellen

4. Feedback geben

Damit diese Interaktionen zwischen einem digitalen Gerät und dem Menschen zu effektiven Lernprozessen führen, unterscheidet Koper (2014) in einem nachfolgenden Schritt zwischen fünf unterschiedlichen HLI-Typen (vgl. Abbildung 2.32), mit jeweils spezifischen Lernzielen (Revidieren \& Restrukturieren, Verbessern \& Wiedergeben, Generieren neuer, kognitiver Repräsentationen) und Lernformen (Reflektieren, Übung, Identifikation, Sozialisation, Kreation).

Koper (2014) argumentiert, dass ausschließlich über tiefe Identifikation mit dem Lerninhalt, soziale Interaktionen und eigene schöpferische Tätigkeiten Verhaltensänderungen im Sinne neuer Repräsentationen im Gehirn ermöglicht werden können und damit zu effektivem Lernen führen (vgl. Abbildung 1.2). Insofern sollten diese Lernformen im Speziellen gefördert werden. 
Im Ergebnis bündelt Koper (2014) die Hauptfunktionen, die ein digitales Gerät innerhalb eines Smart Learning Environments unterstützen sollte (vgl. Abbildung 2.32):

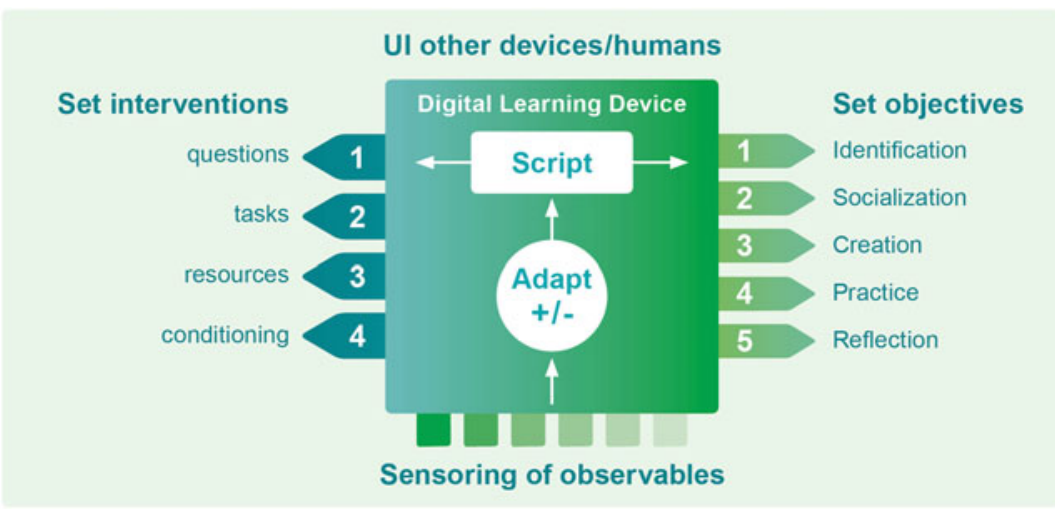

Abbildung 2.32 Hauptfunktionen eines SLE Device (Quelle: Nach Koper 2014)

Auf Grundlage dieser theoretischen Bezüge erweitert Koper seine Definition von Smart Learning Environments wie folgt (Koper, 2014 S. 14):

\begin{abstract}
"SLEs are physical environments that are improved to promote better and faster learning by enriching the environment with context-aware and adaptive digital devices that, together with the existing constituents of the physical environment, provide the situations, events, interventions and observations needed to stimulate a person to learn to know and deal with situations (identification), to socialize with the group, to create artefacts, and to practice and reflect."
\end{abstract}

Das von Koper (2014) vorgestellte HLI Konzept eignet sich in besonderer Weise zur Entwicklung konkreter SLE Use-Cases und der dazu gehörenden ITSystemarchitektur auf mobilen Devices, die beispielsweise über eine App auf einem Smartphone umgesetzt werden könnte. Auf die Bedeutung der Interaktion (im Sinne eines User Interface) zwischen den Lernenden und einem SLE in Form eines digitalen bzw. physischen Assistenten wurde bereits in Abschnitt 2.3.3 eingegangen. Insofern könnte das von Koper ausgearbeitete HLI Konzept z. B. als theoretische Grundlage für die Entwicklung eines Nabaztags (Domingo \& Forner, 2010) genutzt werden. Leider werden elementare und detaillierte Lernformen insbesondere auch im Zusammenspiel mit anderen Lehr- bzw. Lernpersonen 
kaum beleuchtet und nur modellhaft skizziert. Darüber hinaus bleiben Fragen zur Gestaltung des physischen Lernraums offen, der die Lernprozesse stimulieren soll oder aber auch organisationale Rahmenbedingungen und Faktoren, die eine Einführung von SLEs beeinflussen. Diese und weitere unbeantwortete Fragen bündelt Koper (2014) in einer „Research Agenda for SLEs“ in welcher er auf insgesamt sechs Forschungsaspekte hinweist, die im Zusammenhang des HLI Konzeptes noch genauer $\mathrm{zu}$ untersuchen sind.

Tabelle 2.7 Vergleich zwischen ubiquitärem, adaptivem und smartem Lernen (aus dem Englischen nach Hwang 2014)

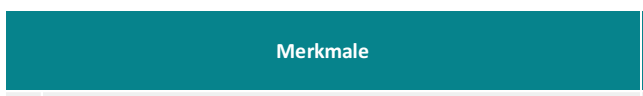

\begin{tabular}{c|c|c}
$\begin{array}{c}\text { ubiquitäres } \\
\text { Lernen }\end{array}$ & $\begin{array}{c}\text { adaptives } \\
\text { Lernen }\end{array}$ & $\begin{array}{c}\text { smartes } \\
\text { Lernen }\end{array}$ \\
\hline
\end{tabular}

1. Erkennt und berücksichtigt den realen Kontext

\begin{tabular}{l|l|l|} 
ja & nein \\
ja & nein
\end{tabular}

3. Passt den Inhalt an unterschiedliche Lernende an

nein $\quad$ ja $\quad$ ja

4. Passt das Interface an unterschiedliche Lernende an

5. Passt Lernaufgaben und Lernziele an individuelle Bedarfe an

nein

ja

ja

ja

6. Bietet persönliches Feedback und Hilfestellung

nein

nein

ja

ja

ja

ja

Bietet Hilfestellung zu Lernmethoden über unterschiedliche Fachdisziplinen hinweg

nein

nein

Bietet Hilfestellung zu Lernmethoden in unterschiedlichen

8. Lernkontexten (Schule, Campus, Bibliothek, Arbeitsplatz, Heimweg, Nahverkehr, Auto etc.)

9. Empfiehlt Lernwerkzeuge und Lernstrategien

10. Beachtet den online-Status der Lernenden

11. Beachtet den real-weltlichen-Status der Lernenden

12. Unterstützt formales und informelles Lernen

13. Berücksichtigt persönliche Faktoren sowie kontextbezogene

Faktoren (Vorlieben, Zeitpläne, Bedürfnisse, Situation etc.)

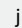

nein

ja

nein

nein

ja

nein

ja

ja

ja

nein

ja

ja

nein

ja

14. Interagiert mit den Nutzern über unterschiedliche (ubiquitous-)

Devices (z.B. Smartphone, VR/AR-Brille etc.)

nein

nein

ja

15.

Unterstützt die Lernenden durch vorangegangene Anpassungen über reale und virtuelle Kontexte hinweg (in Echtzeit)

\begin{tabular}{c|c|c|}
\hline ja & nein & ja \\
\hline nein & nein & ja
\end{tabular}

Hwang (2014) geht in seinem Framework für SLEs zunächst umfassend auf die verwendete Terminologie ein und verweist auf die Verschränkung von adaptiven 
und ubiquitären Lernformen, die in Ihrer Kombination „,smartes Lernen“ ermöglichen (vgl. Abschnitt 2.1.2.5). In Tabelle 2.7 dekonstruiert und vergleicht Hwang (2014) die Begriffe „Smart Learning“, „Ubiquitous Learning“ und „Adaptive Learning“ (vgl. Tabelle 2.7- aus dem Englischen frei übersetzt).

Bei ubiquitärem Lernen ist ein Informationssystem in der Lage den Situationskontext und den Ort der Lernenden zu erfassen und darauf abgestimmte Lernfunktionen zur Verfügung zu stellen. Eine Verschränkung mit adaptivem Lernen bedeutet, dass das System darüber hinaus die Bedürfnisse der Lernenden kennt und auf Grundlage dieser Daten unterschiedliche Informationen und Lernmethoden anbieten kann, die dem jeweiligen Lerntypus entsprechen. Hwang (Hwang, 2014 S. 2) formuliert dies wie folgt:
„A smart learning environment not only enables learners to access digital resources and interact with learning systems in any place and at any time, but also actively provides the necessary learning guidance, hints, supportive tools or learning suggestions to them in the right place, at the right time and in the right form".

Hwang (2014) verweist dabei auf das herausragende Potenzial von ubiquitären Lernformen, die in idealer Weise, der aus der Lehr- und Lernforschung (vgl. Abschnitt 2.1.2.1) geforderten Situiertheit des Lernens gerecht werden können. Hwang fasst demzufolge die Hauptkriterien zusammen, die mindestens in einem SLE repräsentiert sein müssen:

1. Context-Aware: Ein SLE ist kontextbezogen; das heißt die Situation des Lernenden oder die Kontexte der realen Umgebung, in der sich der Lernende aktuell befindet werden wahrgenommen. Dies impliziert, dass das System in der Lage ist, Lernunterstützung auf Grundlage der aktuellen Lernsituation zur Verfügung zu stellen (Online oder Offline)

2. Adaptive: Ein SLE bietet sofortige und adaptive Unterstützung durch Echtzeitanalysen der Bedürfnisse in Abhängigkeit des Lernkontextes

3. Personalized: Ein SLE ist in der Lage, die Benutzerschnittstelle (User Interface) in ihrer Oberflächengestaltung sowie in den darüber zur Verfügung gestellten Inhalte an die persönlichen Bedürfnisse der Lernenden anzupassen.

Auf dieser Grundlage formuliert Hwang (2014, S. 5) folgende Definition von SLEs:

„Smart Learning Environments can be regarded as the technology-supported learning environments that make adaptations and provide appropriate support (e.g., guidance, feedback, hints or tools) in the right places and at the right time based on individual 
learners' needs, which might be determined via analyzing their learning behaviors, performance and the online and realworld contexts in which they are situated.“

Daran anknüpfend zählt Hwang (2014) technische Module auf, die im Rahmen der Gestaltung von SLEs zu modellieren und zu entwickeln sind. Dabei orientiert er sich stark an der Modellierung von Intelligent Tutoring Systems (ITSs), die in der Regel über 4 Module verfügen:

- expert model or expert knowledge model

- student model or learner model

- instructional model or pedagogical knowledge model

- user interface

Im Ergebnis entwickelt Hwang (2014) ein Framework, das aus insgesamt fünf Modulen, einer Reihe Datenbanken sowie einer Inference engine besteht (vgl. Abbildung 2.33):

1. Learning status detecting module:

Erfassung der Umgebung (Standort, Uhrzeit, Temperatur...)

2. Learning performance evaluation module:

Aufzeichnung und Bewertung der Lernergebnisse

3. Adaptive Learning task module:

Zuweisung von Lernaufgaben in Abhängigkeit der persönlichen Lernziele, des Lernfortschritts, der Lernleistung sowie weiterer persönlicher Faktoren

4. Adaptive Learning content module:

Empfehlung von Lerninhalten in Abhängigkeit der aktuellen Situation, der persönlichen Lernziele, des Lernfortschritts, der Lernleistung sowie weiterer persönlicher Faktoren

5. Personal learning support module:

Unterstützung durch Feedback und Hinweise zu Lernstrategien und Methoden

6. Set of databases:

Speicherung von Lernergebnissen, Lernzielen, Lernprofilen und Lernverläufen

7. Inference engine:

Speicherung von Erfahrungen über erfolgreiche und weniger erfolgreiche Lernprozesse für die Lehrenden hinsichtlich kontinuierlicher Verbesserung

Im Vergleich zu den zuvor dargelegten SLE Ansätzen fällt bei Hwang eine stark technisch orientierte Konzeption des SLE Frameworks auf. Man gewinnt den Eindruck, dass sich das Framework überwiegend an Systementwickler richtet. 


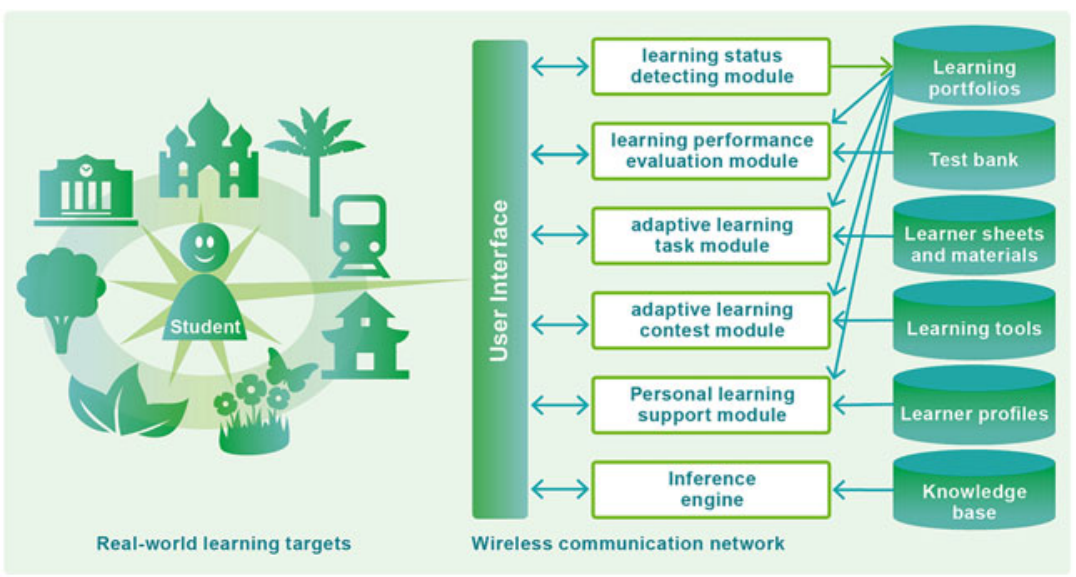

Abbildung 2.33 Smart Learning Environment Framework (Quelle: Nach Hwang 2014)

Entsprechend formuliert Hwang abschließend unterschiedliche Forschungsbedarfe, die sich überwiegend auf pädagogische Fragestellung beziehen. Darüber hinaus verweist er auf die Notwendigkeit, dass SLEs nur in Kooperation mit unterschiedlichen Fachexperten aus unterschiedlichen Fachdisziplinen (insbesondere aus Informatik und Pädagogik) zu entwickeln und zu implementieren sind.

In Ergänzung zu den vorgestellten SLE Konzepten soll abschließend auf den Beitrag von Begoña Gros (2016) eingegangen werden, die durch die Gestaltung von SLEs die Potenziale für selbstgesteuertes Lernen darlegt und für einen partizipativen Gestaltungsprozess von SLEs plädiert.

Nach Gros (2016) haben die starke Verbreitung von mobilen Endgeräten wie dem Smartphone dazu geführt, dass heutzutage überall gelernt werden kann. Darüber hinaus verweist sie in diesem Zusammenhang auf einen selbstbestimmten Umgang der Nutzer*innen mit dem mobilen Gerät. Während in formalen Lernszenarien i. d. R. eine Steuerung durch eine Lehrperson erfolgt, fällt dies beim Lernen mit mobilen Endgeräten weg. Gros formuliert als Ziel von SLEs das Orchestrieren zwischen formalen und informellen Lernformen. Hierfür werden ihrer Meinung nach neue pädagogische Ansätze benötigt, die Lernen als ein subjektives, situiertes, soziales und sehr dynamisches Konstrukt verstehen, das sich bei jedem Lernenden unterschiedlich manifestiert.

Aufbauend auf den nach Zhu et al. (2016) zitierten 10 Eigenschaften von SLEs (1. Location-Aware, 2. Context-Aware, 3. Socially Aware, 4. Interoperability, 5. 
Seamless Connection, 6. Adaptability, 7. Ubiquitous, 8. Whole Record and 9. Natural Interaction) schlussfolgert Gros, dass die Hauptaufgabe von SLEs darin besteht, den Lernenden eine geeignete und passgenaue Lernunterstützung zu bieten. Ein SLE nimmt Gros zufolge also eher die Rolle eines Coachs oder eines Lernbegleiters ein und basiert auf den folgenden pädagogischen Prinzipien:

- Konversation: Die Lernumgebung kann den Lernenden in einen Dialog einbeziehen

- Reflexion: Die Lernumgebung kann eine Selbsteinschätzung basierend auf der Lernleistung vornehmen

- Innovation: Die Lernumgebung nutzt neue und aufkommende Technologien auf kreative Weise

- Selbstorganisation: Die Lernumgebung kann Lerninhalte und Lernmethoden automatisiert ordnen und führt über einen längeren Zeitraum zu verbesserten Lernleistungen

Aufbauend auf Barron's learning ecologies framework (2006) konstatiert Gros, dass die Lernenden die Hauptakteure des smarten Lernens darstellen und selbst verantwortlich sind für soziale Beziehungen und die Schaffung von bedeutsamen Konstellationen im physischen und virtuellen Lernkontext. Demnach plädiert Gros bei der Gestaltung von SLEs für einen menschenzentrierten Ansatz (vgl. Human-Centered Design in Abschnitt 3.3), der gezielt die Bedürfnisse der Lernenden adressiert. Erst wenn man weiß, was „smarte Lernende sind“ und was diese bewegt, können nach Gros sinnvolle SLEs entwickelt werden. Um dieser Forderung gerecht zu werden, verweist Gros direkt zu Beginn auf einen partizipativen Gestaltungsprozess, bei welchem die Fachexperten aus unterschiedlichen Fachdisziplinen gemeinsam mit den Lernenden in Co-Creation agieren. Einem stringent menschenzentrierten Ansatz folgend argumentiert Gros, dass die durch Learning Analytics generierten Erkenntnisse nicht zur Kontrolle durch Dritte, sondern als Tool der Selbstreflektion im Lernprozess genutzt werden sollten und entsprechend nur den Lernenden selbst zur Verfügung stehen sollten. Das Zukunftskonzept der Bildung formuliert Gros (2016, S. 10) als „Education as a Service“ und beschreibt dies wie folgt:

„Services must consider the learners' viewpoint and learning experience. In a smart learning environment, learners would have different service choices at different learning stages, where these services are provided by different educational facilities, either online or physically. Due to the rather blurred lines between formal and informal learning, and the increasing focus on informal learning, it may not be necessary to distinguish these two learning formats separately in the future." 
Es kann festgestellt werden, dass Gros im Vergleich zu den anderen Autoren kein eigenes Konzept zur Gestaltung von SLEs vorlegt, sondern unter Rückbezug auf die aktuelle SLE-Forschung den Prozess der Gestaltung beleuchtet. Im Ergebnis konkludiert Gros, dass die Anwendung von partizipativen Designmethoden dazu beiträgt, SLEs zu entwickeln, die auf die Bedürfnisse und den soziokulturellen Hintergrund der Lernenden eingehen können.

Zusammenfassend ergibt sich ein SLE Forschungsstand, welcher in Funktionalität und Merkmalen überwiegend einheitlich ist. SLEs sind demnach in der Lage, ihre Umwelt zu erkennen und den Lernenden situationsbezogene und bedarfsgerechte Inhalte sowie Lernstrategien und Werkzeuge zur Verfügung zu stellen:

- Detect and take into account the real-world contexts.

- Situate learners in real-world scenarios.

- Adapt learning interfaces for individual learners.

- Adapt learning tasks for individual learners.

- Provide personalised feedback or guidance.

- Provide learning guidance or support across disciplines.

- Provide learning guidance or support across contexts.

- Recommend learning tools or strategies.

- Consider learners' online learning status.

- Consider learners' real-world learning status.

- Facilitate both formal and informal learning.

- Take multiple personal and environmental factors into account.

- Interact with users via multiple channels.

- Provide learners with support in advance, across real and virtual contexts.

Zudem besteht weitgehend Einigkeit in der Notwendigkeit, SLEs disziplinübergreifend zu erforschen und zu entwickeln, indem insbesondere pädagogische und informationstechnische Erkenntnisse miteinander verbunden werden können.

Auffällig ist jedoch der divergierende Zugang der einzelnen Autoren, der sich darin äußert, dass die vorgestellten Konzepte auf unterschiedliche Ebenen ausgerichtet sind, konzeptionelle Vorgehensweisen voneinander abweichen und auch in den theoretischen Bezügen variieren. Nichtsdestotrotz stellen die dargelegten Ansätze, Konzepte und Frameworks zur Gestaltung von SLEs relevante Erkenntnisse zur Verfügung, auf die im Rahmen der vorliegenden Arbeit aufzubauen sind.

Im nachfolgenden Abschnitt erfolgt anknüpfend an die Darstellung des SLE Forschungsstands die Herleitung der Forschungslücken. 


\subsubsection{Herleitung der Forschungslücken}

Die Darstellung des aktuellen Forschungsstands zu SLEs diente der Herleitung und der Untermauerung der in Kapitel 1 bereits skizzierten Forschungslücken. Anhand bestehender Literatur konnten in Kapitel 2 inhaltliche Fragestellung in Bezug zum Untersuchungsgegenstand erläutert sowie dessen interdisziplinären Bezüge abgeleitet werden. Im folgenden Kapitel werden die Forschungsfragen dahingehend operationalisiert, um sie empirisch untersuchbar zu machen. Auf dieser Grundlage konnten folgende Forschungslücken identifiziert werden:

Umfassende Literaturrecherchen haben ergeben, dass das Thema „IoT-basierter Bildungsformate" ein bisher unbeachtetes Forschungsthema ist. Eine dedizierte Erforschung von Anwendungsmöglichkeiten auf Lehr- und Lernprozesse gibt es weder in Deutschland noch im internationalen Forschungsdiskurs. Entsprechend ist bisher unklar, ob das Internet der Dinge zu neuen Lernformaten führen kann und wie diese zu bezeichnen wären. Für den deutschsprachigen Raum konnte darüber hinaus festgestellt werden, dass sich die Publikationen zum Thema Internet der Dinge im Bildungskontext ausschließlich damit beschäftigen, welche IoTKompetenzen in Zukunft benötigt werden und wie diese strategisch aufgebaut werden können. Ein Mangel an empirischen Befunden verweist zudem darauf, dass die Durchführung von Studien überwiegend ausgeblieben ist, obwohl das Internet der Dinge in den letzten Jahren deutlich an Interesse gewonnen hat.

Eine Literaturrecherche auf Basis sekundärer IoT-Suchbegriffe hat zu der Erkenntnis geführt, dass Smart Learning Environments die englische Bezeichnung für intelligente und hybride Lernräume sind, wobei auch eine Bedeutungsgleichheit nachgewiesen werden konnte. Doch obwohl es sich in der SLE-Literatur um physische Lernräume handelt, werden architektonische Gestaltungsprinzipien nicht adressiert. Darüber hinaus gibt es Defizite hinsichtlich organisationaler Aspekte und Rahmenbedingungen, die eine Implementierung von SLEs beeinflussen. Insgesamt kann festgestellt werden, dass es an einem ganzheitlichen, interdisziplinären und soziotechnisch ausgerichteten Konzept fehlt, das darüber hinaus ein systematisches und wissenschaftlich fundiertes Verfahren zur Gestaltung von SLEs liefert.

Tabelle 2.8 zeigt zusammenfassend die im Rahmen der Literaturrecherche identifizierten Lücken. Zusätzlich wird darstellt, durch welche Forschungsfragen (vgl. Unterkapitel 1.2.2) diese adressiert werden. In dem folgenden Kapitel 3 werden die Forschungsfragen mit Hilfe empirischer Erhebungen sowie qualitativer und quantitativer Methoden sukzessive bearbeitet, um in Kapitel 4 beantwortet zu werden. 
Tabelle 2.8 Identifizierte Forschungslücken

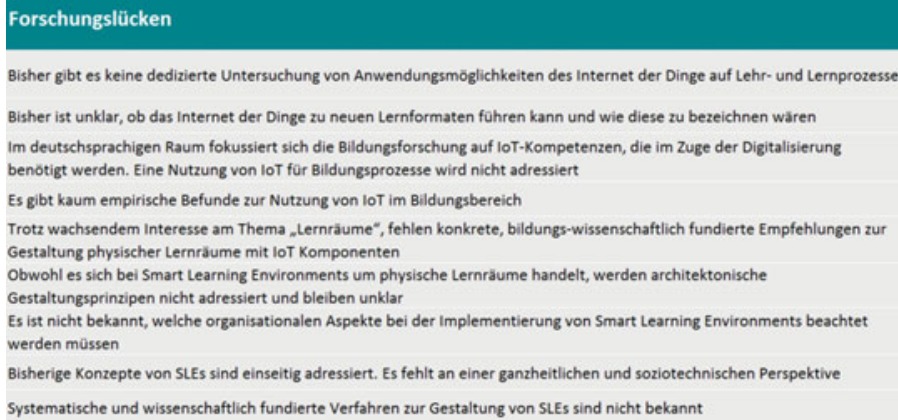

\subsection{Schlussfolgerungen aus dem interdisziplinären Forschungsansatz}

Die transdisziplinären Literaturanalysen haben ergeben, dass einheitliche und voneinander abgrenzbare Definitionen der Begriffe „Ubiquitäres Lernen“, „Adaptives Lernen“ und „Smartes Lernen“ je nach Perspektive unterschiedlich ausfallen. Der Vergleich vorhandener Definitionen lässt dennoch den Rückschluss zu, dass sich durch die Übertragung von IoT auf Lehr- und Lernprozesse das sogenannte „Smarte Lernen“ innerhalb von Smart Learning Environments von ubiquitärem und adaptivem Lernen abgrenzen lässt und entsprechend mehr ist, als die Kombination von adaptivem und ubiquitärem Lernen. Diese Interpretation basiert auf den vergleichenden Merkmalen aus Tabelle 2.7. Demnach konnte gezeigt werden, dass durch die Übertragung von IoT auf Lehr- und Lernprozesse ein neues Lernformat entsteht, das unter dem Begriff Smart Learning Environments gefasst werden kann.

Darüber hinaus wurde deutlich, dass für eine Übertragung von IoT auf Lehrund Lernprozesse die physische Lernumgebung, also Lernräume ein wichtiges Gestaltungselement darstellt, da das IoT physische und digitale Welten miteinander verbindet. Im Ergebnis resultieren hybride Lernräume, in welchen physische und digitale Lernformen verschmelzen und fließend ineinander übergehen.

Wie in den vorhergehenden Abschnitten ausführlich dargestellt, wirkt sich das Internet der Dinge zunehmend auf Organisationen und den damit verbundenen Lern- und Arbeitsprozessen aus. Die Arbeitswelt befindet sich in einem Wandel. Langfristige und übergreifende Transformationsprozesse wie die Digitalisierung, Globalisierung oder die Wissensbasierte Ökonomie - kurz die Megatrends des 
21. Jahrhunderts sind Indikatoren einer massiven Umstrukturierung. Infolge des Strukturwandels, von der Industriegesellschaft in eine Wissensgesellschaft verändern sich auch die Tätigkeitsfelder und somit die Anforderungsprofile der Erwerbstätigen. Damit einher geht das notwendige Erlernen neuer Systeme, Prozesse, Umgangs- und Arbeitsformen, die als Aspekte einer lernenden Organisation zum ausschlaggebenden Wettbewerbsfaktor werden. Nicht zuletzt steht der Kulturwandel als Leitwort für einen menschzentrierten Denkansatz, welcher neben dem technologisch bedingten Wandel auch die Bedürfnisse der Mitarbeiter*innen adressiert, um dualen Anforderungen an einen zukunftsorientierten Arbeitsplatz gerecht zu werden.

Die Arbeitswelt der Zukunft ist mehr denn je eine Symbiose aus Mensch und Maschine. Der Kostendruck verleitet Organisationen immer stärker auf Maschinen und Automatisierung zu setzen. Gleichzeitig sehen sich die Organisationen damit konfrontiert, sich das dafür notwendige Wissen über die Maschinen anzueignen. Dazu gehören jedoch nicht nur maschinelle Funktionsweisen, sondern auch das Innovieren neuer Einsatzszenarien. Dort, wo es auf Kreativität statt auf Maschinenlaufzeit ankommt, versagt ein auf quantitativen Größen basierender Begriff von Arbeit. Wenn sich der Arbeitsbegriff ändert, muss sich auch unsere Vorstellung von Lernen dem Prüfstand stellen. Im Bereich der Aus- und Weiterbildung muss verstärkt über die Rolle von Kreativität und Innovationen nachgedacht werden. Innovation wird letztlich als zentraler Treiber für das 21. Jahrhundert gesehen. Aus diesem Grund werden Innovationsmethoden wie z. B. Design Thinking wie in einem Hype zelebriert, verbunden mit der Hoffnung, damit entsprechend für die Zukunft gerüstet zu sein. Massive Veränderungen sind jedoch nicht allein in den Arbeits- und Lernmethoden vorzufinden, sondern sie erstrecken sich auf die kompletten Wertschöpfungsprozesse. Von daher werden neue und ganzheitlich ausgerichtete Strategien benötigt, die gleichzeitig auf mehreren Ebenen ansetzen.

In den aktuellen bildungswissenschaftlichen Diskursen wird deutlich, dass die Digitalisierung zu einer komplexer werdenden Bildungsarbeit führt. Folglich ist es empfehlenswert, die Arbeitsteilung stärker als bisher zwischen Partnern mit sehr speziellen Kompetenzen zu organisieren, bei der beispielsweise Spezialisten für Diagnostik, für die Entwicklung von digitalen Lernumgebungen, für die Lernprozessbegleitung sowie für das Testing und Analytics zusammenarbeiten. Gerade in einem hybriden Zusammenwirken aller im Lernprozess involvierten Phasen und Formen, die vorhandene (Medien-) Brüche überwinden und alle Elemente in der Wertschöpfungskette neu kombiniert, wird sich vermutlich die Transformation der Bildungsarbeit zeigen. Diesem Ansatz folgend verbindet die vorliegende 
Forschungsarbeit drei Fachdisziplinen mit dem Ziel, ein ganzheitliches, soziotechnisches Gestaltungsverfahren für eine zukunftstaugliche Bildungspraxis zu liefern.

Im Zentrum dieser bildungstheoretischen Transformationsprozesse stehen Menschen, die die Bildung neu denken und innovieren. Es ist nicht die Technik, die die Bildung verändert. Nichtsdestotrotz eröffnet das Internet der Dinge neue Zugänge des Lernens, die durch Smart Learning Environments ein enormes Potenzial für arbeitsplatzbezogene Lernformen erkennen lassen. Um diesen Wandlungsprozess theoretisch fundiert zu begleiten ist es wichtig, alle in diesem Kapitel dargestellten Anforderungen aus einer pädagogischen, technischen und architektonisch/designbasierten Perspektive zu betrachten, um auf einer fundierten theoretischen Basis eine spezifizierte Gestaltungsarbeit beginnen zu können. Erst in der holistischen Betrachtung des Lernraumes, der sich durch eine materielle, virtuelle und soziale Ebene manifestiert, wird es möglich, Smart Learning Environments didaktisch fundiert konzipieren zu können. Zur Komplexitätsreduktion wurde die in diesem Kapitel hergeleitete theoretische Basis in ein Modell überführt, das die wichtigsten Anforderungen zur Gestaltung von SLEs bündelt, um einen Transfer der wissenschaftlichen Erkenntnisse in die Praxis zu ermöglichen (vgl. Abschnitt 3.4).

Open Access Dieses Kapitel wird unter der Creative Commons Namensnennung 4.0 International Lizenz (http://creativecommons.org/licenses/by/4.0/deed.de) veröffentlicht, welche die Nutzung, Vervielfältigung, Bearbeitung, Verbreitung und Wiedergabe in jeglichem Medium und Format erlaubt, sofern Sie den/die ursprünglichen Autor(en) und die Quelle ordnungsgemäß nennen, einen Link zur Creative Commons Lizenz beifügen und angeben, ob Änderungen vorgenommen wurden.

Die in diesem Kapitel enthaltenen Bilder und sonstiges Drittmaterial unterliegen ebenfalls der genannten Creative Commons Lizenz, sofern sich aus der Abbildungslegende nichts anderes ergibt. Sofern das betreffende Material nicht unter der genannten Creative Commons Lizenz steht und die betreffende Handlung nicht nach gesetzlichen Vorschriften erlaubt ist, ist für die oben aufgeführten Weiterverwendungen des Materials die Einwilligung des jeweiligen Rechteinhabers einzuholen.

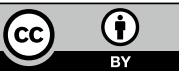

